\title{
Making the EGSnrc/BEAMnrc system more efficient, accurate and realistic in simulating kilovoltage $x$-ray systems
}

by

\section{Elsayed S. M. Ali}

\author{
A thesis submitted to the \\ Faculty of Graduate Studies and Research \\ in partial fulfillment of the requirements \\ for the degree of \\ Master of Science \\ Department of Physics \\ Carleton University \\ Ottawa-Carleton Institute of Physics \\ Ottawa, Canada
}

September 20, 2007

Copyright (C) 2007 Elsayed S. M. Ali 


$\begin{array}{ll}\begin{array}{l}\text { Library and } \\ \text { Archives Canada }\end{array} & \begin{array}{l}\text { Bibliothèque et } \\ \text { Archives Canada }\end{array} \\ \begin{array}{l}\text { Published Heritage } \\ \text { Branch }\end{array} & \begin{array}{l}\text { Direction du } \\ \text { Patrimoine de l'édition }\end{array} \\ \begin{array}{l}\text { 395 Wellington Street } \\ \text { Ottawa ON K1A ON4 }\end{array} & \begin{array}{l}\text { 395, rue Wellington } \\ \text { Ottawa ON K1A ON4 } \\ \text { Canada }\end{array}\end{array}$

Your file Votre référence ISBN: 978-0-494-33689-2 Our file Notre référence ISBN: 978-0-494-33689-2

NOTICE:

The author has granted a nonexclusive license allowing Library and Archives Canada to reproduce, publish, archive, preserve, conserve, communicate to the public by telecommunication or on the Internet, loan, distribute and sell theses worldwide, for commercial or noncommercial purposes, in microform, paper, electronic and/or any other formats.

The author retains copyright ownership and moral rights in this thesis. Neither the thesis nor substantial extracts from it may be printed or otherwise reproduced without the author's permission.
AVIS:

L'auteur a accordé une licence non exclusive permettant à la Bibliothèque et Archives Canada de reproduire, publier, archiver, sauvegarder, conserver, transmettre au public par télécommunication ou par l'Internet, prêter, distribuer et vendre des thèses partout dans le monde, à des fins commerciales ou autres, sur support microforme, papier, électronique et/ou autres formats.

L'auteur conserve la propriété du droit d'auteur et des droits moraux qui protège cette thèse. $\mathrm{Ni}$ la thèse ni des extraits substantiels de celle-ci ne doivent être imprimés ou autrement reproduits sans son autorisation.
In compliance with the Canadian

Privacy Act some supporting forms may have been removed from this thesis.

While these forms may be included in the document page count, their removal does not represent any loss of content from the thesis.
Conformément à la loi canadienne sur la protection de la vie privée, quelques formulaires secondaires ont été enlevés de cette thèse.

Bien que ces formulaires aient inclus dans la pagination, il n'y aura aucun contenu manquant.

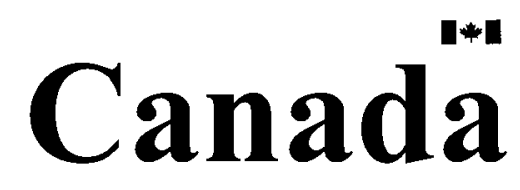




\section{Abstract}

This project aims to improve the performance of the EGSnrc/BEAMnrc system for kilovoltage medical physics applications, particularly for simulation of $\mathrm{x}$-ray systems. This involves making the simulations more efficient, more accurate and more realistic.

For efficient simulations, a variance reduction technique is newly implemented which improves the efficiency of simulations involving bremsstrahlung targets by up to five orders of magnitude over analog simulations, and by up to a full order of magnitude over the state of the art of the system without the newly added technique. For accurate simulations, a comprehensive and stringent benchmark of the system against experimental measurements of charged particle backscatter shows excellent agreement between EGSnrc results and most of the experimental data. For realistic simulations, the ability to simulate voltage ripple and offfocal radiation are both added to the system. Other new features have also been introduced. 


\section{Acknowledgments}

To my advisor, Prof. Dave Rogers: I would like to express my sincere gratitude and appreciation for giving me the opportunity to learn from you. Your knowledge, friendliness and availability are the most I could have ever asked for in an advisor. Your workaholism is contagious though!

To Prof. Paul Johns: thank you for the valuable consultations on the technical aspects of $\mathrm{x}$-ray tubes. I owe you for referring me to the importance of modeling the off-focal radiation. Also, your rigorousness has benefited me a lot (in your class, you took half a point off because I wrote $\mathrm{keV}$ with a capital $\mathrm{K}$ !).

I would like to extend my thanks to Prof. Tong Xu and Prof. John Boone for consultations on x-ray tubes, Dr. Iwan Kawrakow at NRC for consultations on EGSnrc, Randy Taylor for lots of personal and computer coding help, Dan La Russa for his collaboration in developing hvl_clrp, and the other members of the Carleton Laboratory for Radiotherapy Physics for embracing me when I first joined the group, and for their helpful comments on my manuscripts. I also would like to thank Dr. Joanna Cygler for her warm welcoming of me to Canada, Olaf Nairz for his valuable help with the German articles for the backscatter benchmark, WestGrid for letting me use their nodes for some of my calculations, and the Physics Department at Carleton University for financial support and for the sense of family it made me feel.

To my parents: thank you for all that you have endured throughout the years for me. I owe any success in my life to you. To my wife, Azza: thank you for your love, support and understanding. I am lucky to have you in my life.

This thesis is dedicated to my mother. 


\section{Statement of originality}

The contents of this thesis is a summary of the author's work during the course of his M.Sc. program at Carleton University. The contents have been published in, or submitted to, various scientific journals. Parts of the contents have also been presented at national and international conferences. The following is a summary of the literature upon which this thesis is based. Except for item 7 in the list below, the author of this thesis performed all the calculations and drafted and edited all the manuscripts.

\section{Peer-reviewed full papers}

1. E. S. M. Ali and D. W. O. Rogers, Efficiency improvements of x-ray simulations in EGSnrc user-codes using Bremsstrahlung Cross Section Enhancement (BCSE), Med. Phys. 34, 2143 - 2154, (2007). ${ }^{1}$

2. E. S. M. Ali and D. W. O. Rogers, Benchmarking EGSnrc in the kilovoltage range against experimental measurements of charged particle backscatter coefficient, Phys. Med. Biol., to be submitted (September 2007). ${ }^{2}$

3. E. S. M. Ali and D. W. O. Rogers, Energy spectra and angular distribution of charged particles backscattered from solid targets, J. Phys. D: Appl. Phys., to be submitted (September 2007). ${ }^{3}$

4. E. S. M. Ali and D. W. O. Rogers, Effect of off-focal (extra-focal) radiation on the output parameters of x-ray tubes, Med. Phys., in preparation (2007). ${ }^{4}$

\section{Oral conference presentations with presenting author: E. S. M. Ali}

5. E. S. M. Ali and D. W. O. Rogers, More efficient simulation of bremsstrahlung targets in EGSnrc user-codes, $3^{\text {rd }}$ McGill Int. Conf. on Monte Carlo Techniques in Radiotherapy Delivery and Verification, Montreal, Quebec, May/June 2007.

6. E. S. M. Ali and D. W. O. Rogers, Off-focal radiation in x-ray tubes: An EGSnrc/ BEAMnrc investigation, Accepted for the Joint CARO-COMP Annual Scientific Meeting on Image-Guided and Adaptive Radiation Therapy, Toronto, Ontario, October 2007.

\section{Other oral conference presentations}

7. D. W. O. Rogers, G. Yegin, R. E. P. Taylor and E. S. M. Ali, Fast Monte Carlo dose calculations for brachytherapy with seeds, HDR and x-ray sources, European Workshop on Monte Carlo Treatment Planning, Ghent, Belgium, October 2006 (presenting author: D.W.O. Rogers). 


\section{Contents}

1 Introduction 1

1.1 The EGSnrc/BEAMnrc system . . . . . . . . . . . . . 1

1.2 Variance reduction techniques ................ . 3

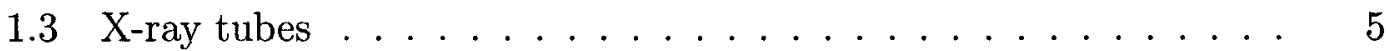

1.4 Kilovoltage simulations in EGSnrc . . . . . . . . . . . . . 10

1.5 Thesis overview . . . . . . . . . . . . . . . . 12

2 Improving simulation efficiency for kilovoltage applications $\quad 14$

2.1 Introduction . . . . . . . . . . . . . . . . . . . 14

2.2 Implementing BCSE in EGSnrc user-codes . . . . . . . . . . . 18

2.3 Benchmarking BCSE . . . . . . . . . . . . . 20

2.4 Results .............................. 22

2.4.1 BCSE without UBS or DBS ............ 22

2.4.2 BCSE in $4 \pi$ geometry (brachytherapy) . . . . . . . 25

2.4.3 BCSE in directional geometry (diagnostic, mammography and orthovoltage tubes) . . . . . . . . . 27

2.4.4 BCSE with clinical linear accelerators . . . . . . . 31

2.5 Discussion . . . . . . . . . . . . . . . . 32

2.6 Summary . . . . . . . . . . . . . . . . . . . 38

3 Benchmarking EGSnrc for kilovoltage application 39

3.1 Introduction . . . . . . . . . . . . . . . . . 39

3.2 Definitions ...................... 41

vi 
3.3 Experimental data $\ldots \ldots \ldots \ldots \ldots \ldots$

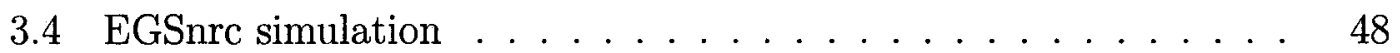

3.5 Results . . . . . . . . . . . . . . . . 52

3.5.1 Total backscatter coefficient . . . . . . . . . . . 52

3.5 .2 Energy spectra . . . . . . . . . . . . . . . 58

3.5.3 Angular distribution . . . . . . . . . . . . . 63

3.5.4 Backscatter from thin films . . . . . . . . . . 70

3.5.5 Positron backscatter . . . . . . . . . . . . 74

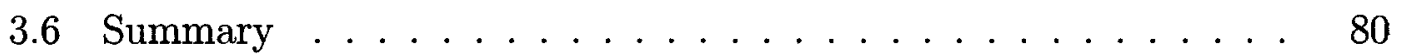

4 Off-focal radiation (OFR) 81

4.1 Introduction $\ldots \ldots \ldots \ldots \ldots \ldots \ldots$

4.2 Implementing OFR in BEAMnrc $\ldots \ldots \ldots \ldots \ldots . \ldots$

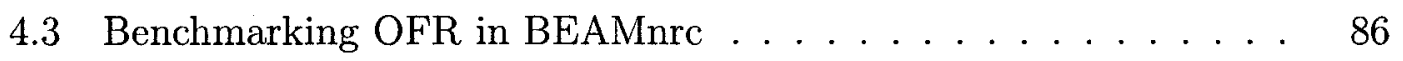

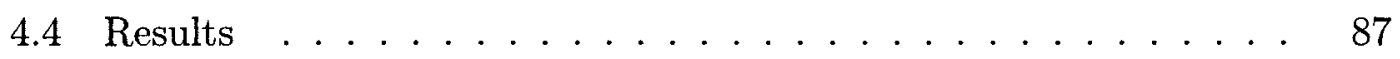

4.4.1 First-generation backscatter . . . . . . . . . . 87

4.4.2 Higher generation backscatter . . . . . . . . . . . . 91

4.4.3 Effect of OFR on output parameters of x-ray tubes . . . 94

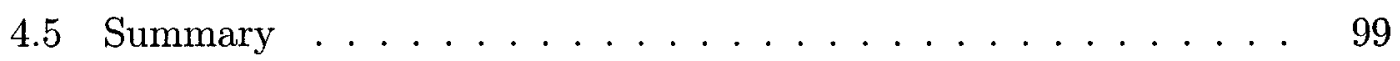

$\begin{array}{lll}5 & \text { Future work } & 100\end{array}$

6 Conclusions 102

$\begin{array}{ll}\text { Appendix A } & 105\end{array}$

$\begin{array}{ll}\text { A OFR implementation } & 105\end{array}$

A.1 Relativistic kinematics of electron motion . . . . . . . . . . . 105

A.2 Transport of backscattered electrons . . . . . . . . . 109

$\begin{array}{ll}\text { References } & 112\end{array}$ 


\section{List of Tables}

2.1 BCSE gains in 4- $\pi$ geometries (brachytherapy) . . . . . . . . . 27

2.2 BCSE gains in directional geometries (diagnostic, mammography and orthovoltage tubes) . . . . . . . . . . 30

2.3 BCSE gains with clinical linear accelerators . . . . . . . . 33

2.4 Recommended optimum cross section enhancement factors . . . . 36

2.5 CPU time for $2 \%$ uncertainty on fluence for optimized simulations 37

3.1 Definition of backscatter parameters . . . . . . . . . . . 43

3.2 Index of experimental data used in the benchmark study . . . . . 44

4.1 Multiple generation-backscatter coefficient for typical situations of x-ray tube usage . . . . . . . . . . . . . . 92 


\section{List of Figures}

1.1 Examples of possible uses of EGSnrc/BEAMnrc in simulations of x-ray tubes ........................ 12

2.1 Paucity of bremsstrahlung emission in the kilovoltage range . . . . 15

2.2 Benchmarking BCSE versus an analog simulation . . . . . . . 21

2.3 Performance of BCSE without UBS or DBS . . . . . . . . . 23

2.4 Performance of BCSE versus UBS in $4 \pi$ geometry . . . . . . . . . 26

2.5 Sensitivity of maximum efficiency to $f_{\text {enh }}$ and $N_{\text {split }} \ldots \ldots . . .35$

2.6 Example of a spectrum obtained from a one-minute optimized simulation ....................... 38

3.1 Definition of angles and planes used in the benchmark study . . . 42

3.2 Effect of boundary tolerance on the accuracy of backscatter coefficient calculation in BEAMnrc . . . . . . . . . . . . 51

3.3 EGSnrc results versus experimental data for the variation of electron backscatter coefficient with the incident electron energy . . . 54

3.4 EGSnrc results versus experimental data for the variation of electron backscatter coefficient with the atomic number of the target

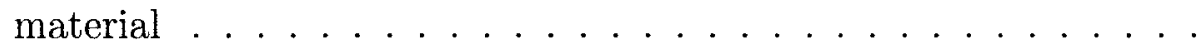

3.5 EGSnrc results versus experimental data for the variation of electron backscatter coefficient with the angle of incidence . . . . . 57

3.6 EGSnrc results versus experimental data for the overall energy spectra of backscattered electrons . . . . . . . . . . . .

3.7 EGSnrc results versus experimental data for the variation of the energy spectra of backscattered electrons with the angle of incidence, and with the angle of backscatter ............ . 
3.8 EGSnrc results versus experimental data for the most probable and the mean energy of backscattered electrons . . . . . . . . . .

3.9 EGSnrc results versus experimental data for the angular distribution of backscattered electrons . . . . . . . . . . . . .

3.10 EGSnrc results versus experimental data for the variation of the angular distribution of backscattered electrons with the atomic number of the target material . . . . . . . . . . . . . .

3.11 EGSnrc results versus experimental data for the variation of the angular distribution of backscattered electrons with the angle of incidence

3.12 EGSnrc results versus experimental data for the variation of the angular distribution of backscattered electrons with the plane of

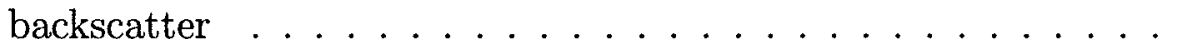

3.13 Backscatter from thin films: EGSnrc results versus experimental data for the variation of electron backscatter coefficient with the incident electron energy . . . . . . . . . . . .

3.14 Backscatter from thin films: EGSnrc results versus experimental data for the energy spectra and angular distribution of backscattered electrons $\cdot \cdot \cdot \cdot \cdot \cdot \cdot \cdot \cdot \cdot \cdot \cdot \cdot \cdot \cdot \cdot \cdot \cdot \cdot \cdot \cdot \cdot \cdot \cdot \cdot \cdot \cdot \cdot \cdot$

3.15 EGSnrc results versus experimental data for the variation of total positron backscatter coefficient with the incident positron energy, with the angle of incidence, and with the atomic number of the target material

3.16 EGSnrc results versus experimental data for the variation of the ratio of electron-to-positron total backscatter coefficient with the incident charged particle energy, and with the atomic number of the target material . . . . . . . . . . . . . . . .

3.17 EGSnrc results versus experimental data for the variation of the angular distribution of backscattered positrons with the angle of incidence . . . . . . . . . . . . . . . .

4.1 Example of OFR . . . . . . . . . . . . . 82

4.2 Variation of the lateral displacement of first generation backscattered electrons with the cathode-anode separation . . . . . . . .

4.3 First generation backscatter coefficient for typical x-ray target ele-

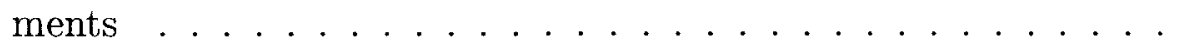

4.4 Energy spectra and angular distribution of first generation backscattered electrons for typical situations of x-ray tube usage . . . . . . 
4.5 Lateral displacement of different generations of backscattered electrons ..................... . . . 92

4.6 Energy spectra and angular distribution of multiple-generation backscattered electrons for typical situations of x-ray tube usage . 93

4.7 Effect of OFR on the output spectrum of an x-ray tube . . . . . . 95

4.8 Effect of OFR on x-ray tube output in terms of air kerma, HVL and effective energy, for various $\mathrm{kVp} \ldots . \ldots 96$

4.9 Effect of OFR on x-ray tube output in terms of air kerma, HVL and effective energy, for various added filtration . . . . . . . 


\section{Chapter 1}

\section{Introduction}

\subsection{The EGSnrc/BEAMnrc system}

Monte Carlo techniques are widely used in simulating coupled photon and charged particle transport problems. In a Monte Carlo simulation, a large number of initial particles (histories) is tracked throughout various geometries and media. During the simulation, individual particle trajectories are determined by using pseudorandom numbers to sample from the probability density functions that describe the underlying physical processes. The output of a simulation represents the average behavior of the tracked particles, and the variance of the reported output is inversely proportional to the number of histories simulated.

$E_{\text {GSnrc }}^{5}$ (Electron-Gamma Shower) is one of the most commonly used Monte Carlo codes in medical physics. It contains the core physics and supporting data required to accurately simulate photon and charged particle transport in various media. All EGSnrc user-codes use the same core EGSnrc physics, but they feature different geometries and score different quantities. $B E A M n r c^{6}$ is a widely-used EGSnrc user-code; its geometry package is particularly suitable for simulating 
clinical linear accelerators, but it can be used for many other applications. As a convention in this thesis, the term EGSnrc system is used when discussing core physics issues, the term BEAMnrc system is used when discussing user-code specific issues not related to the physics, and the term EGSnrc/BEAMnrc system is used when discussing issues that are either coupled or applicable to both the user-code BEAMnrc and its underlying EGSnrc core.

The EGSnrc system uses the condensed history, ${ }^{7}$ or multiple-scattering, technique to simulate parts of the charged particle transport. The technique groups the effect of a large number of charged particle interactions into one condensed step. This is because charged particles undergo a large number of elastic collision with the nuclei of the transport media, and simulating those collisions individually makes simulation times prohibitively long. Interactions that are not included in the condensed steps are treated explicitly as discrete interactions. Kawrakow ${ }^{7}$ proved that his implementation of the condensed history technique in the EGSnrc system yields results that are step-size independent, and that agree, within less than $0.1 \%$, with the results obtained using explicit charged particle treatment. The terminology introduced in this paragraph will be used throughout this thesis.

The EGS system started as a high energy physics tool, ${ }^{8}$ but over the years many features have been added to accurately model the low energy physics of photon and charged particle transport. For photons, the additions relevant to kilovoltage applications include: Rayleigh scattering, binding effects in Compton scattering, photoelectron angular sampling, and relaxation cascades after creation of atomic vacancies. For charged particles, the additions relevant to kilovoltage applications include: an accurate and artifact-free algorithm for charged particle transport, ${ }^{7}$ electron impact ionization ${ }^{9}$ (whereby electrons directly create vacancies in the inner shells of the medium atoms), spin effects for electron elastic scattering,

\subsection{THE EGSNRC/BEAMNRC SYSTEM}


improved bremsstrahlung angular sampling, and improved treatment of inelastic collisions. In addition to the accurate physics, the EGSnrc system uses NIST tabulations for photon cross sections ${ }^{10}$ and for differential bremsstrahlung cross sections ${ }^{11,12}$ which are the most accurate data available. For more details on the EGSnrc physics and supporting data, the reader is referred to the EGSnrc manual ${ }^{5}$ and the references therein. For all kilovoltage simulations performed throughout this project, the low energy physics mentioned above is always included, and NIST tabulations are used for photon and charged particle cross sections (including density effects).

\subsection{Variance reduction techniques}

One of the issues associated with Monte Carlo simulations is the potentially large CPU time required to reach the desired statistics on the scored quantities, even with the dramatic increase in the speed of computers in the past two decades. To get around this issue, variance reduction techniques (VRTs) are often used. These techniques artificially manipulate the simulations to avoid spending much CPU time in aspects of the simulation that do not contribute much to the quantities of interest. If a true VRT is implemented properly, it does not alter the simulation results. ${ }^{13}$ A Monte Carlo simulation that does not employ any VRT is called an analog simulation. The merit of any given VRT is judged by the amount of time saving compared to an analog simulation for the same variance. This method of evaluation is heavily used in chapter 2 of this thesis.

Every particle in a Monte Carlo simulation has a statistical weight $W$ (initially unity). When VRTs are used, the weight of the particle is adjusted to keep the results unbiased. An example, which is used in chapter 2 of this thesis, is playing Russian Roulette with a particle. This is done with particles that are not expected

\subsection{VARIANCE REDUCTION TECHNIQUES}


to have a large impact on the scored quantities. A random number between 0 and 1 is sampled; if it is larger than a certain fraction (called the survival probability), the particle history is terminated; however, if it is less than the survival probability, the particle is kept and its weight is increased from $W$ to $W /$ survival probability. The surviving particle then represents all the other eliminated particles, and for a large enough number of histories, the results are not biased.

To improve the efficiency of simulations that involve bremsstrahlung production (e.g. simulation of x-ray tubes), two VRTs are made available in the EGSnrc/BEAMnrc system: Uniform Bremsstrahlung Splitting (UBS) and Directional Bremsstrahlung Splitting (DBS). UBS was introduced in the original EGS4 ${ }^{14} /$ BEAM $^{15}$ system. In UBS, after a bremsstrahlung photon is generated, it is split into $N_{\text {split }}$ (a user-defined value) photons, each with a reduced weight of $1 / N_{\text {split }}$, then all the $N_{\text {split }}$ photons are tracked in all directions. UBS works best in $4 \pi$ geometry simulations (e.g. brachytherapy) because all the tracked splitphotons contribute to the scored quantity (e.g. dose to voxels). On the other hand, $\mathrm{DBS}^{16}$ was introduced more recently in the BEAMnrc system. The basic concept of splitting in DBS is the same as in UBS. However, in DBS, only the split photons that are directed towards the field of interest are tracked, while Russian Roulette is played with the ones directed away from the field of interest. DBS works best in directional geometry simulations (e.g. diagnostic tubes and medical linear accelerators) because the field of interest subtends a small solid angle, and tracking only the photons directed towards that small solid angle substantially reduces the simulation time. Because UBS and DBS are very heavily quoted in chapter 2 of this thesis, the reader is encouraged to be sure to absorb the material just presented.

\subsection{VARIANCE REDUCTION TECHNIQUES}




\section{$1.3 \quad$ X-ray tubes}

$\mathrm{X}$ rays can penetrate the human body and attenuate with varying degrees depending on the materials encountered in their paths. The operation of the tube that generates these $\mathrm{x}$ rays is as follows: The two electrodes of the tube are called the cathode and the anode. The cathode contains a thoriated tungsten wire, called the filament which heats up and discharges electrons by thermionic emission when current ( $\sim 3-5$ A) passes through it. The anode is typically a disk made of tungsten or molybdenum, alloyed with rhenium or rhodium for strength. A high voltage $(\sim 20-140 \mathrm{kV})$, called the $k V p$, is applied between the cathode and the anode, with the anode as the positive end. The electrons emitted by the cathode are accelerated towards the anode causing a tube current ( $50-1200 \mathrm{~mA}$ ). A negatively-charged focusing cup surrounds the filament to redirect most electrons towards the anode. The accelerated electrons hit the anode at the focal spot and undergo elastic and inelastic collisions with the nuclei and electrons of the target material. Radiative collisions with the target nuclei produce a continuous bremsstrahlung spectrum, whereas relaxations after atomic vacancy creation produce characteristic peaks. The output of an x-ray tube is the combination of the bremsstrahlung spectrum and the characteristic peaks.

X-ray tubes are very inefficient energy conversion devices; only $\sim 1 \%$ of the incident energy is converted into $\mathrm{x}$ rays, and the remaining $\sim 99 \%$ is converted into heat. For that reason, rotating anodes $(\sim 3000 \mathrm{rpm})$ are typically used to avoid melting of the target at the focal spot. The cathode and anode are kept in vacuum in a sealed glass insert. The glass is thinner in the portion facing the focal spot, making the tube exit window where the generated $\mathrm{x}$ rays get out of the tube towards the patient plane.

\subsection{X-RAY TUBES}


Generated $\mathrm{x}$ rays go through multiple stages of filtration where lower energy $\mathrm{x}$ rays are preferentially absorbed due to the higher cross section of the photoelectric effect at lower energies. This causes beam hardening which is needed to avoid unnecessary surface dose to the patient. The first filtration, self filtration, is experienced within the target itself. This is followed by the inherent filtration by the glass (or sometimes beryllium) window. The tube output can further be filtered (hardened) using added filtration. Immediately outside the tube exit window, primary collimators are used to limit the $\mathrm{x}$ rays to a controlled useful cone. This cone of $x$ rays can be further collimated down the path of $x$ rays towards the patient plane. This last step of collimation is done using diaphragms, jaws or applicators to form diagnostic or therapeutic fields of particular sizes and/or shapes.

For $\mathrm{x}$ rays to get out of the tube towards the patient plane, the target is tilted by a relatively small angle $\left(\sim 6-24^{\circ}\right)$ relative to the line perpendicular to the patient plane. This causes $\mathrm{x}$ rays that penetrate deeper into the target and still reach the patient plane to be, on average, fewer in number and higher in energy than those closer to the surface. This is called the heel effect, which can be seen when the fluence or the energy fluence is recorded in the patient plane along a line parallel to the cathode-anode axis. The heel effect is either made use of (by positioning the thinner body parts in the lower energy end and the thicker body parts in the higher energy end), or compensated for (by using compensating filters which are shaped to produce more uniform spectra once the tube output passes through them).

To reduce the distortion in the image caused by the component of $\mathrm{x}$ rays scattered within the imaged body part, anti-scatter grids are commonly used. They are composed of alternating septa of high-Z (lead) and low-Z (air, paper

\subsection{X-RAY TUBES}


or plastic) materials, typically focused towards the tube focal spot. Anti-scatter grids allow the primary $\mathrm{x}$-ray component to pass through the low- $\mathrm{Z}$ material while the scattered component (which is not lined up with the focusing line between the $\mathrm{x}$-ray source and the anti-scatter grid) is absorbed by the high- $\mathrm{Z}$ material. In practice, focused grids are more frequently used than other types (parallel or cross grids). As part of this project, the BEAMnrc component module MLC (which is designed to simulate multi-leaf collimators in clinical linear accelerators) is modified to be able to simulate parallel, focused and cross grids. The modified component module needs to be benchmarked against experimental measurements. For brevity, this feature is not discussed any further in this thesis.

By convention, there are three main energy ranges in which x-ray tubes are used for medical applications. They are: the mammography range $(\sim 20-40 \mathrm{kVp})$, the diagnostic range $(\sim 60-140 \mathrm{kVp})$, and the orthvoltage range $(\sim 160-300 \mathrm{kVp})$. Mammography systems (used for breast imaging) typically have molybdenum targets, small target angles ( $\sim$ a few degrees), beryllium windows, $\sim 30 \mu$ m molybdenum added filtration, and an $18 \times 24 \mathrm{~cm}^{2}$ imaging field at $65 \mathrm{~cm}$ SSD. Diagnostic systems (used for imaging most body parts) typically have tungsten targets, target angles $\sim 7-10^{\circ}$ for Computed Tomography $(\mathrm{CT})$ and $\sim 10-20^{\circ}$ for conventional radiography, thin glass windows, $2-5 \mathrm{~mm}$ aluminum equivalent added filtration, and imaging field sizes ranging from $10 \times 10$ up to $40 \times 40 \mathrm{~cm}^{2}$ at $100 \mathrm{~cm}$ SSD depending on the body part being imaged. Orthovoltage systems (used for treatment of superficial cancers) typically have tungsten targets, larger target angles $\left(\sim 16-24^{\circ}\right)$, hooded anodes, thin glass windows, up to $\sim 1 \mathrm{~mm}$ copper equivalent added filtration, and therapeutic field sizes ranging from $2 \times 2$ up to $15 \times 15 \mathrm{~cm}^{2}$ at $52 \mathrm{~cm}$ SSD depending on the skin portion or the body part being treated. In this thesis, although mammography is technically a diagnostic $\mathrm{x}$-ray application, it is

\subsection{X-RAY TUBES}


separated from the higher $\mathrm{kVp}$ applications because of the difference in the energy range used, and because of the distinct features of mammography systems (e.g. different target material). With this in mind, the phrase "diagnostic, mammography and orthovoltage systems", which is commonly used throughout this thesis, should not confuse the reader.

There are many ways to analyze the output of an $\mathrm{x}$-ray tube and to compare simulation results with experimental measurements. The spectrum at the patient plane is a very common way to represent the tube output. A second way is the collision air-kerma, $\left(K_{c}\right)_{a i r}$, or simply air kerma, $K_{a i r}$ (because radiative losses in air are negligible). $K_{a i r}$ is the summation over all energy bins of the energy fluence spectrum, weighted by the mass energy absorption coefficient of air. $K_{a i r}$ replaces the older, readily measurable quantity exposure, $X$. A third way is the Half Value Layer, HVL, which is the thickness of a particular absorber required to reduce $K_{a i r}$ (or $X$ ) to half its value. HVLs are commonly expressed in millimeters of aluminum for mammography and diagnostic applications, and in millimeters of copper for orthovoltage applications. A fourth way, which is closely-related to HVL, is the effective energy of the spectrum, $E_{\text {eff }}$, defined as the energy of a monoenergetic $\mathrm{x}$-ray beam that has the same HVL as the spectrum. As part of this project, a new user-code (hvl_clrp) is developed (in collaboration with D. La Russa) to calculate $K_{a i r}, H V L$ and $E_{e f f}$ given an energy fluence spectrum at the patient plane and an absorber material. The code employs the following equations for the calculations.

- For $K_{\text {air }}$

$$
K_{a i r}=\sum_{i=1}^{n} \frac{d \Psi_{E_{i}}}{d E_{i}}\left(\frac{\mu_{e n}}{\rho}\right)_{E_{i}}^{a i r}
$$


- For $H V L$ : The absorber thickness, $t$, is iterated until the following equation ${ }^{17}$ is satisfied (at which point $t=H V L$ ):

$$
\frac{K_{a i r}(t)}{K_{a i r}(0)}=\frac{\sum_{i=1}^{n}\left(\frac{\mu_{e n}}{\rho}\right)_{E_{i}}^{a i r} \frac{d \Psi_{E_{i}}}{d E_{i}} \exp \left(-\left(\mu_{E_{i}}^{a b s}-\mu_{E_{i}}^{a i r}\right) t\right) \Delta E_{i}}{\sum_{i=1}^{n}\left(\frac{\mu_{e n}}{\rho}\right)_{E_{i}}^{a i r} \frac{d \Psi_{E_{i}}}{d E_{i}} \Delta E_{i}}=\frac{1}{2}
$$

Here, $K(0)$ and $K(t)$ are the air kerma at the patient plane without the absorber, and with an absorber of thickness $t$, respectively. $E_{i}$ is the energy at the center of bin $i, \Delta E_{i}$ is the width of bin $i, n$ is the number of energy bins, $\left(\frac{\mu_{e n}}{\rho}\right)_{E_{i}}^{a i r}$ is the mass energy absorption coefficient for air at energy $E_{i}, \mu_{E_{i}}^{a b s}$ and $\mu_{E_{i}}^{a i r}$ are the linear attenuation coefficients at energy $E_{i}$ for the absorber material and for air, respectively, and $\frac{d \Psi_{E_{i}}}{d E_{i}}$ is the differential energy fluence at energy $E_{i}$.

- For $E_{\text {eff }}$ : When equation 1.2 is applied for monoenergetic x rays that give the same HVL as the spectrum, and neglecting the thickness of the air substituted by the absorber in the exponent of the attenuation term, then $n=1, t=H V L$, $E_{i}=E_{e f f}$, and $\mu_{E_{i}}^{a b s}=\mu_{E_{e f f}}^{a b s}$. Solving for $\mu_{E_{e f f}}^{a b s}$ :

$$
\mu_{E_{e f f}}^{a b s}=\frac{\ln 2}{H V L}
$$

Then hvl_clrp searches the linear attenuation coefficient table for the absorber material to get the energy $E_{e f f}$ at which $\mu_{E_{i}}^{a b s}=\mu_{E_{e f f}}^{a b s}$.

Older models of $\mathrm{x}$-ray tubes use single or three phase AC generators. This means that the high voltage applied between the cathode and the anode is variable. The percent voltage ripple is used to quantify this variation. The percent ripple in single- and three phase generators (which are still being used in research and in clinical practice) is $100 \%$ and $13 \%$, respectively. Mechanical oscillations and other operational parameters cause further enhancement of the percent ripple.

\subsection{X-RAY TUBES}


Even with the most recent $\mathrm{x}$-ray systems that use high frequency generators, the ripple is still $\sim 5 \%$. For more realistic simulation of $\mathrm{x}$-ray tubes, voltage ripple effects need to be included. As part of this project, voltage ripple effects are added in BEAMnrc. Simulation results with and without voltage ripple are qualitatively benchmarked against semi-empirical models that simulate voltage ripple. ${ }^{18}$ The trends are similar for comparisons of spectra, air-kerma, HVLs and effective energies. More quantitative benchmarks are needed, and the effect of the $\mathrm{V} / \mathrm{I}$ characteristic of the tube on the percent voltage ripple need to be taken into account. Ultimately, the user will have the option to either provide a V/I curve for the particular tube and operational parameters simulated, or use a set of default $\mathrm{V} / \mathrm{I}$ curves supplied by BEAMnrc which represent typical $\mathrm{x}$-ray tubes and typical operational parameters. For brevity, this feature is not discussed any further in this thesis.

The concepts discussed in this section, and the four ways to analyze the output of an x-ray tube are all used throughout this thesis. The user-code hvl_clrp is used in section 4.4.3 to study the effect of the off-focal radiation component on the output parameters of $\mathrm{x}$-ray tubes.

\subsection{Kilovoltage simulations in EGSnrc}

There has been a growing interest in using the EGSnrc/BEAMnrc system (and its predecessors) in a wide variety of medical physics applications in the kilovoltage range, particularly in the simulation of x-ray tubes, ${ }^{1,17,19-30}$ and in dosimetry. ${ }^{31-34}$ This growing interest can be attributed to the accurate low energy physics in the system, the multitude of Variance Reduction Techniques (VRTs) available in EGSnrc and its user-codes, the system's adoption of the most accurate photon and charged particle cross sections, the increasing clinical trend of integrating

\subsection{KILOVOLTAGE SIMULATIONS IN EGSNRC}


kilovoltage imaging units with most therapy systems, and finally, the increase in the computing power per unit cost which makes Monte Carlo more appealing than ever. The detailed simulations of kilovoltage systems are essential for better assessment of image quality in diagnostic x-ray imaging, for investigation of challenges encountered in kilovoltage applications, for better evaluation of patient entrance absorbed-dose, and for better regulation of the standards in diagnostic imaging, radiation therapy and dosimetry.

This project uses BEAMnrc to simulate kilovoltage x-ray systems. The geometry package of BEAMnrc has many component modules that can be used to accurately represent the various components of an x-ray system. The specific component modules used in this project are: XTUBE for the x-ray target and backing, CONESTAK and FLATFILT for primary collimation and for applicators in orthovoltage tubes, CHAMBER for monitor ion chamber, JAWS for secondary collimation to create diagnostic fields, MLC and MESH for anti-scatter grids, and SLABS for various components such as the inherent filtration, the added filtration, the cover layers of an anti-scatter grid, ... etc. BEAMnrc allows the user to investigate many different output parameters of the x-ray tube system including: fluence distribution at the patient plane, energy spectra, energy fluence, angular distribution, mean energy, ... etc. The flexibility of the system allows the user to answer questions that may be hard to answer experimentally. Figure 1.1 shows an example of possible uses of the EGSnrc/BEAMnrc system in simulations of x-ray system.

1.4. KILOVOLTAGE SIMULATIONS IN EGSNRC 

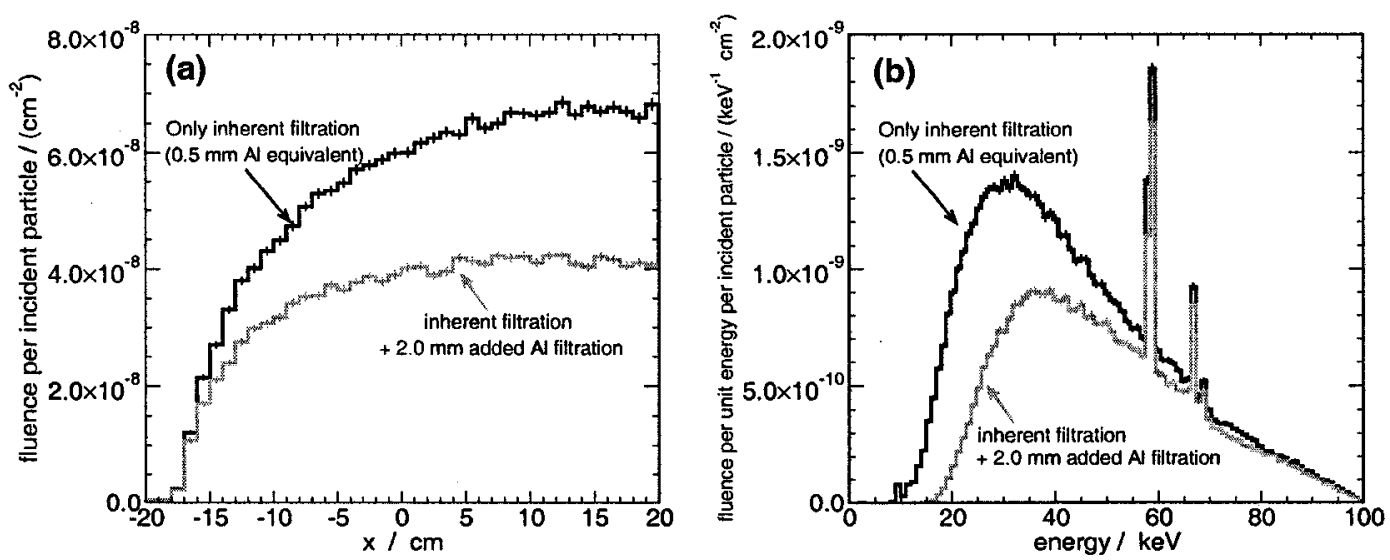

Figure 1.1: Examples of possible uses of EGSnrc/BEAMnrc in simulations of $x$-ray tubes. Specifications in the examples are: $100 \mathrm{kV} \mathrm{DC}$ diagnostic tube, $10^{\circ}$ angle $\mathrm{W}$ target, $\mathrm{Cu}$ backing, $0.5 \mathrm{~mm} \mathrm{Al}$ equivalent inherent filtration. Panel a: Fluence profiles showing the heel effect along the cathode-anode direction, $x$, in a $40 \times 40$ $\mathrm{cm}^{2}$ field at $100 \mathrm{~cm} \mathrm{SSD}$, with and without added Al filtration. Panel b: energy spectra for pinhole geometry (opening of radius $0.5 \mathrm{~cm}$ at $100 \mathrm{~cm}$ SSD on the central axis) with and without added Al filtration.

\subsection{Thesis overview}

This thesis is organized as follows:

- Chapter 2 presents the "efficient simulations" aspect of the project. The chapter describes the idea behind the newly-added VRT, and how it has the potential to address the challenges facing the existing VRTs. Next, implementation and benchmarking are briefly highlighted. The Results section shows the efficiency gains obtained when optimally combining the newly-added technique with the existing ones. Finally, an easy two-step algorithm for simulation optimization is described. 
- Chapter 3 presents the "accurate simulations" aspect of the project. The chapter describes a comprehensive benchmark of the EGSnrc/BEAMnrc system using backscatter measurements. The experimental measurements, their associated uncertainties and their respective EGSnrc simulations are described. This is followed by presentation and analysis of the benchmark results for many backscatter parameters, and for a variety of operational conditions and measurement setups.

- Chapter 4 presents the "realistic simulations" aspect of the project. The chapter describes the implementation and benchmarking of off-focal radiation in BEAMnrc, followed by a presentation of the characteristics of multiple generations of electrons backscattering from x-ray tube targets. The chapter concludes with a study of the effect of including off-focal radiation on the output parameters of $\mathrm{x}$-ray tubes.

- Chapter 5 highlights possible extensions to the project, which could lead to even further improvements in the EGSnrc/BEAMnrc system for kilovoltage medical physics applications.

- Chapter 6 summarizes the overall conclusions from the project.

- The Appendix is used to develop some equations needed to implement the contribution of off-focal radiation in BEAMnrc simulations (appendices are pertinent to chapter 4).

Finally, it should be noted that this chapter has provided a general introduction for only the topics common to all parts of the thesis. More specific introductions are included at the beginning of every part of the project. 


\section{Chapter 2}

\section{Improving simulation efficiency for kilovoltage applications}

\subsection{Introduction}

The efficiency, $\varepsilon$, of a Monte Carlo simulation is defined as:

$$
\varepsilon=\frac{1}{T s^{2}}
$$

where $T$ is the simulation CPU time and $s^{2}$ is an estimate of the statistical variance on the quantity of interest. Using this definition of simulation efficiency, analog Monte Carlo simulations involving bremsstrahlung targets operating in the kilovoltage range are very inefficient. This is because the probability of bremsstrahlung emission from electrons decelerating in the target material is very small, which means that most of the CPU time is consumed in tracking electrons to a stop without their giving off any photons. A Monte Carlo study is carried out in which a stream of monoenergetic electrons impinges onto a thick bremsstrahlung target, and the total number of bremsstrahlung events is counted 


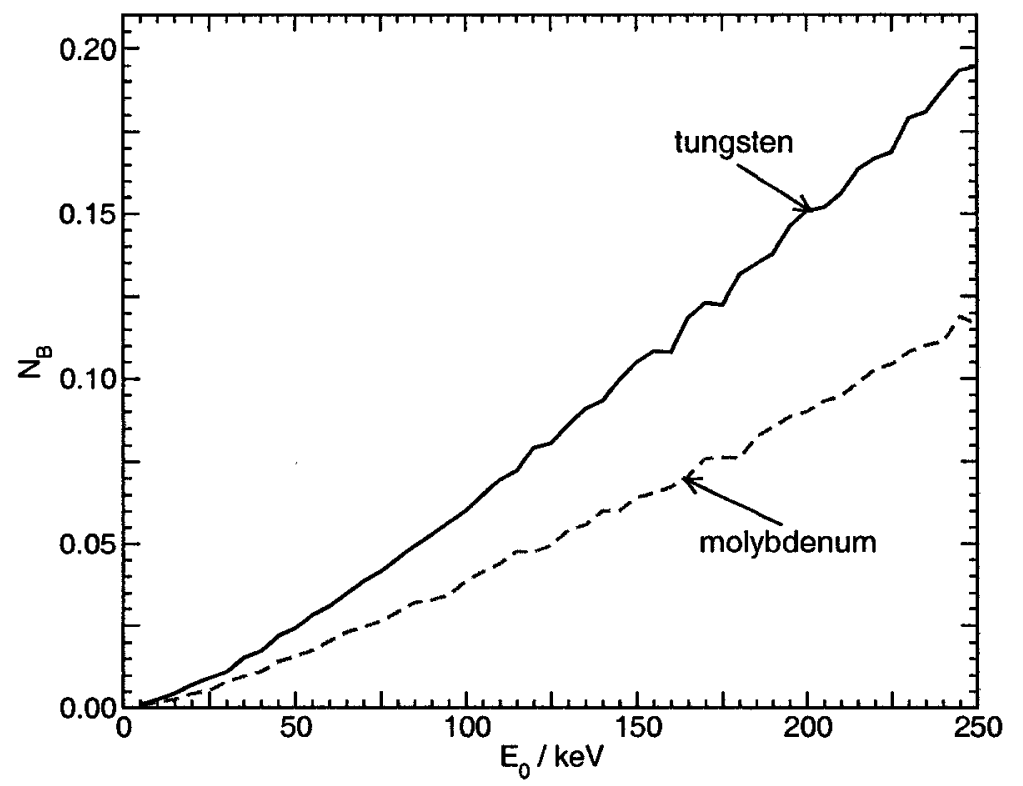

Figure 2.1: The average number of bremsstrahlung photons, $N_{B}$, emitted by an electron slowing down from kinetic energy $E_{0}$ to $1 \mathrm{keV}$ inside thick tungsten and molybdenum targets, as a function of the initial electron kinetic energy $E_{0}$.

and averaged over the total number of histories. Figure 2.1 shows the results for both tungsten and molybdenum targets. On average, the probability of emission of a bremsstrahlung photon per incident electron is less than $10 \%$ for tungsten in the diagnostic range (around $100 \mathrm{keV}$ ) and less than $0.5 \%$ for molybdenum in the mammography range (around $20 \mathrm{keV}$ ). Even in the range of orthovoltage tubes (160-250 keV), the probability does not exceed $20 \%$.

To overcome this inefficiency of analog Monte Carlo simulation of bremsstrahlung targets, radiation transport Monte Carlo codes based on ETRAN ${ }^{35}$ and PENELOPE ${ }^{36,37}$ have included an option to enhance the production of bremsstrahlung photons and characteristic radiation if desired (called interaction forcing). However, to the author's knowledge, there have not been any studies that optimized the use of this option or quantified the efficiency gains from implementing it in typical situations of interest in the medical physics field. In addition, the 
EGSnrc/BEAMnrc system does not have this option, and including it would be a valuable addition, particularly for kilovoltage simulation. This part of the project both implements the Bremsstrahlung Cross Section Enhancement (BCSE) variance reduction technique in the EGSnrc/BEAMnrc system, and also quantifies the associated efficiency gains from using it in typical situations of interest in diagnostic $\mathrm{x}$-ray imaging and in radiotherapy.

The basic idea of the BCSE technique is to scale up the bremsstrahlung production cross section by a factor $f_{\text {enh }}$ everywhere in the target material, then some aspects of the simulation are re-worked to keep the results unbiased; such aspects include reducing the weight of the resulting bremsstrahlung photons in the target material by a factor of $1 / f_{\text {enh }}$, and randomly decrementing the energy of the charged particle once every $f_{\text {enh }}$ times of bremsstrahlung emission. In other words, the energy of the charged particle is not decremented every time a reduced weight bremsstrahlung photon is emitted in the target material. This ensures that there is no bias that produces more photons near the target surface and disturbs the various features of the $\mathrm{X}$-ray tube output (such as the heel effect or the energy spectrum). It also ensures that the charged particle follows the same path it would have followed without BCSE; the only difference is that it gives off more reduced weight bremsstrahlung photons everywhere (not only close to the surface). Generation of those statistically-independent extra photons reduces the fractional CPU time spent in tracking the charged particles, and hence increases the simulation efficiency. The benchmarking section in this chapter will unequivocally confirm that $\mathrm{BCSE}$ is a true variance reduction technique and that it does not introduce any bias in the simulation.

2.1. INTRODUCTION 
Despite the large efficiency gains reported when the existing splitting techniques in EGSnrc/BEAMnrc (viz. Uniform Bremsstrahlung Splitting and Directional Bremsstrahlung Splitting) are optimized, ${ }^{16,17}$ there remain four issues. (1) Both splitting techniques will not come into play until the rare event of bremsstrahlung production happens in the first place. (2) EGSnrc uses a historyby-history statistical estimator ${ }^{38}$ for the uncertainty on the scored quantity, which takes into account correlation between scored particles arising from the same incident particle. Since all $N_{\text {split }}$ photons are correlated when using UBS or DBS, efficiency gains saturate then drop once these correlated particles start falling into the same scoring zone or voxel. ${ }^{39}$ (3) The largest efficiency gains when using DBS are achieved for small field sizes (pinhole geometry for example), ${ }^{17}$ but these gains decrease dramatically as the fields of interest get larger (e.g. a $40 \times 40 \mathrm{~cm}^{2}$ diagnostic field). (4) The only currently available variance reduction technique in EGSnrc for $4 \pi$ geometry is UBS, whose efficiency gains are not as impressive as those for DBS, and boosting them is very desirable. Combining BCSE with UBS/DBS alleviates the limitations caused by the four issues discussed above because BCSE reduces the number of histories that do not generate bremsstrahlung photons, it creates more statistically-independent photons that push the efficiency gains of UBS and DBS further, and it works equally well for small field sizes, large field sizes and $4 \pi$ geometry.

Although the main scope of the BCSE technique is the kilovoltage range, where bremsstrahlung production is rarest, its implementation in this part of the project is made for the entire energy range. Both electron and positron bremsstrahlung emission is dealt with, and the term charged particle applies to both electrons and positrons.

2.1. INTRODUCTION 


\subsection{Implementing BCSE in EGSnrc user-codes}

In EGSnrc, cross sections and other data are read from an input data file at the beginning of the simulation. ${ }^{5}$ For electron cross sections, the input data file includes the total discrete interaction cross section $\sigma_{\text {total_original }}$, and the branching ratio $B_{\text {brem_original }}$ for the fraction of discrete interactions that are bremsstrahlung events. To enhance the bremsstrahlung cross section for a user-requested medium by a user-requested enhancement factor $f_{e n h}$, the bremsstrahlung cross section is scaled to $\sigma_{\text {brem_enhanced }}$ and the total cross section and the bremsstrahlung branching ratio are adjusted to their new values $\sigma_{\text {total_new }}$ and $B_{\text {brem_new }}$ as follows:

$$
\begin{aligned}
\sigma_{\text {Moller }} & =\sigma_{\text {total_original }}\left(1-B_{\text {brem_original }}\right) \\
\sigma_{\text {brem_enhanced }} & =\sigma_{\text {total_original }} B_{\text {brem_original }} f_{\text {enh }} \\
\sigma_{\text {total_new }} & =\sigma_{\text {Moller }}+\sigma_{\text {brem_enhanced }} \\
B_{\text {brem_new }} & =\sigma_{\text {brem_enhanced }} / \sigma_{\text {total_new }}
\end{aligned}
$$

where $\sigma_{\text {Moller }}$ is the discrete electron-electron inelastic scattering cross section. Then the two parameters esig_e and esige_max (which are used in specific sampling algorithms during the electron transport in EGSnrc) are also adjusted using the new total cross section $\sigma_{\text {total_new }}$ (esig_e and esige_max are the maximum electron cross section per unit restricted stopping power for the medium enhanced and for all media, respectively). For positrons, in addition to the quantities mentioned above for electrons, the input data file includes a second branching ratio, $B_{(b r e m+B h a b h a) \_o r i g i n a l}$, for the fraction of discrete interactions that are either bremsstrahlung or Bhabha events. The bremsstrahlung cross section enhancement for positrons proceeds as follows:

\subsection{IMPLEMENTING BCSE IN EGSNRC USER-CODES}




$$
\begin{aligned}
& \sigma_{\text {annih+Bhabha }}=\sigma_{\text {total_original }}\left(1-B_{\text {brem_original }}\right) \\
& \sigma_{\text {Bhabha }}=\sigma_{\text {total_original }}\left(B_{(b r e m+B h a b h a) \_o r i g i n a l}-B_{b r e m \_o r i g i n a l}\right) \\
& \sigma_{\text {brem_enhanced }}=\sigma_{\text {total_original }} B_{\text {brem_original }} f_{\text {enh }} \\
& \sigma_{\text {total_new }}=\sigma_{a n n i h+B h a b h a}+\sigma_{\text {brem_enhanced }} \\
& B_{\text {brem_new }}=\sigma_{\text {brem_enhanced }} / \sigma_{\text {total_new }} \\
& B_{(b r e m+B h a b h a) \_n e w}=\left(\sigma_{b r e m_{-} \text {enhanced }}+\sigma_{\text {Bhabha }}\right) / \sigma_{\text {total_new }}
\end{aligned}
$$

where $\sigma_{B h a b h a}$ is the discrete positron-electron inelastic scattering cross section and $\sigma_{\text {annih }}$ is the discrete positron-electron annihilation cross section. Then, as done for electrons, psig_e and psige_max are adjusted using the new total cross section $\sigma_{\text {total_new }}$ (psig_e and psige_max are the maximum positron cross section per unit restricted stopping power for the medium enhanced and for all media, respectively).

The simplest case of BCSE implementation is when it is not combined with either UBS or DBS. In this case, and for a cross section enhancement factor $f_{\text {enh }}$, when bremsstrahlung photons are produced in the enhanced medium, their weight $W$ (initially unity) is reduced to $W / f_{e n h}$ to counteract the effect of cross section enhancement. In addition, a uniform random number between 0 and 1 is sampled, and if it is larger than $1 / f_{\text {enh }}$, the energy of the charged particle is kept at its value before the bremsstrahlung event took place; otherwise the energy of the charged particle is decremented by the amount of energy given to the bremsstrahlung photon. This means that the emission of a bremsstrahlung photon does not necessarily cause a reduction in the charged particle energy. This is done to avoid altering the physics of the charged particle transport in the enhanced medium.

\subsection{IMPLEMENTING BCSE IN EGSNRC USER-CODES}


Second-generation charged particles are created through photoelectric, Compton and pair-production interactions of first-generation low weight photons. This triggers higher generations of vanishingly low weight photons and charged particles that, if tracked, will consume CPU time without contributing much to reducing the variance of the scored quantity. In addition, fat photons (i.e. photons of weight $W$ ) are created through relaxation events after electron impact ionization (both in the enhanced medium and elsewhere), through bremsstrahlung events outside the enhanced medium, and through positron annihilation events. If these fat photons reach the field of interest, they degrade the statistics of the scored quantity. The complex details of how the BCSE algorithm handles higher order low weight photons and large weight fat photons can be found in Ali and Rogers. ${ }^{1}$

When BCSE with a cross section enhancement factor $f_{e n h}$ is combined with either UBS or DBS with a splitting number $N_{\text {split }}$, many subtle details need to be considered in order to avoid weight variation issues that degrade the statistics. Again, these details are described in Ali and Rogers. ${ }^{1}$ In total, for all the cases involving BCSE, all the photons that reach the field of interest at the scoring plane have the same statistical weight.

\subsection{Benchmarking BCSE}

BCSE is a true variance reduction technique; if it is implemented properly, it should not bias any scored quantity. To ensure its proper implementation, rigorous benchmarking tests are performed on all the simulations presented in section 2.4 before they are used to study the performance of the BCSE technique. Other cases are also benchmarked to test particular issues. Parameters such as spectral distribution, fluence profiles (where the heel effect can be seen for angled targets) and three dimensional dose maps are used for comparison. A detailed list of the

\subsection{BENCHMARKING BCSE}




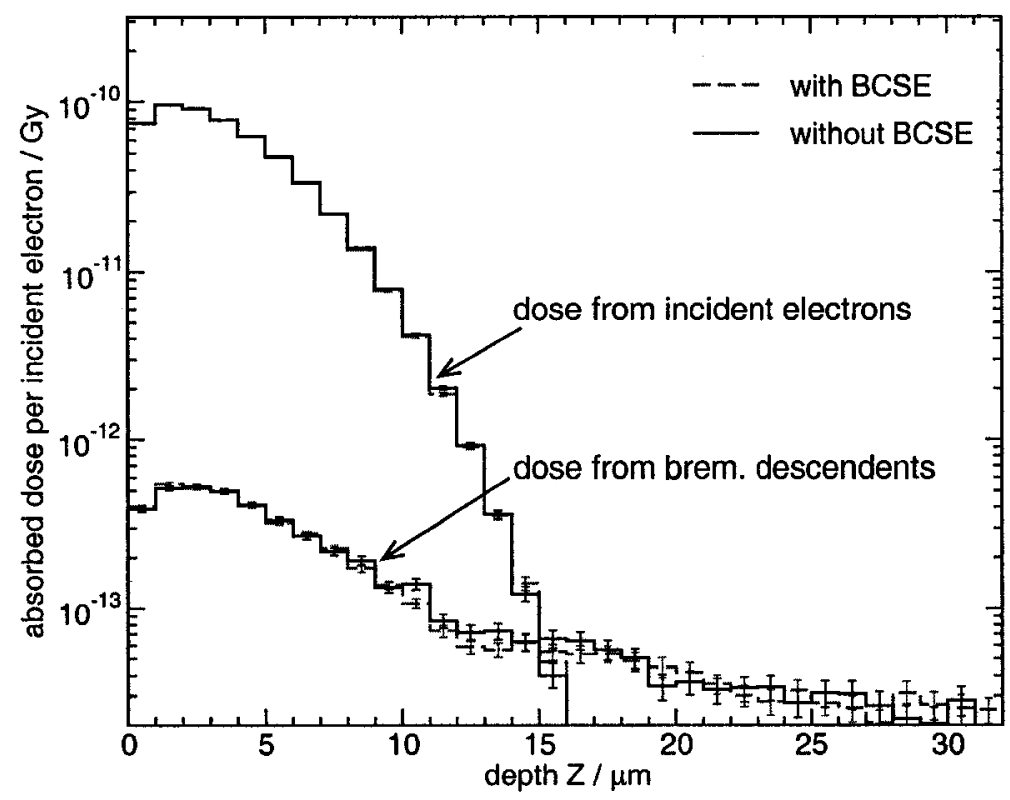

Figure 2.2: Depth dose distribution for $130 \mathrm{keV}$ electrons normally incident on a tungsten target. Dose is scored inside the target around the central axis in small cylinders of $5 \mathrm{~mm}$ radius and $1 \mu \mathrm{m}$ thickness each. Dose is split into two components: a component from direct energy deposition by the incident electrons, and a component from energy deposition by the charged particles descending from bremsstrahlung photons generated within the target.

benchmarks performed is given in Ali and Rogers. ${ }^{1}$ Figure 2.2 shows the results of one of the most stringent tests performed. The graph confirms that the energy loss by the charged particles along their tracks is the same, within statistics, with and without BCSE. It also shows that the extra photons generated by enhancing the bremsstrahlung cross section are generated everywhere along the charged particle track, not only closer to the surface, and that their energy and angular distribution is the same as it is without BCSE. Similar agreement is obtained for all other benchmarking tests, within $0.5 \%$, for energy spectra, fluence profiles and dose maps. Passing such rigorous tests confirms that the BCSE technique is a true variance reduction technique, and that it has been implemented correctly in EGSnrc/BEAMnrc. It also shows that the BCSE technique is compatible with the other variance reduction techniques in EGSnrc/BEAMnrc.

\subsection{BENCHMARKING BCSE}




\subsection{Results}

In studying the performance of the BCSE technique, emphasis is put on typical situations encountered in the clinical use of bremsstrahlung targets, both in diagnostic x-ray imaging and in radiotherapy, in order for the reported efficiency gains to be of greatest practical use. Simulations are done using two EGSnrc user-codes: BrachyDose $^{40}$ and a customized version of BEAMnrc. ${ }^{6}$ BrachyDose scores dose by calculating the collision kerma using a track length estimator of fluence, weighted by the mass energy absorption coefficient. The customized BEAMnrc version allows for grid scoring within the field of interest and reports the sum of the variance for all the scoring zones within the grid, which is then used as a measure of the variance $s^{2}$ in equation 2.1 for efficiency calculations. The most accurate physics and cross sections available in EGSnrc are included. For kilovoltage simulations, electrons and photons are tracked down to $1 \mathrm{keV}$. A rejection plane is introduced with DBS to prevent fat photons from interacting in the air layer just above the scoring plane and degrading the statistics of the scored quantities. Simulations show that eliminating those fat photons has a negligible effect on fluence scoring in the kilovoltage range for typical field sizes of interest.

\subsubsection{BCSE without UBS or DBS}

Although BCSE is meant to be used in combination with UBS or DBS to boost their efficiency gains, it is instructive to study the behavior of simulation efficiency versus $f_{e n h}$ without UBS or DBS. The diagnostic x-ray tube described in section 2.4.3 below is used for that purpose. Four scoring grids are used on a $40 \times 40 \mathrm{~cm}^{2}$ field at $100 \mathrm{~cm}$ SSD; each grid has equal square scoring zones $(1 \times 1$, $2 \times 2,4 \times 4$ and $8 \times 8 \mathrm{~cm}^{2}$ ). The cross section enhancement factor, $f_{\text {enh }}$, is varied between 1 (no cross section enhancement) and 1,000,000.

2.4. RESULTS 


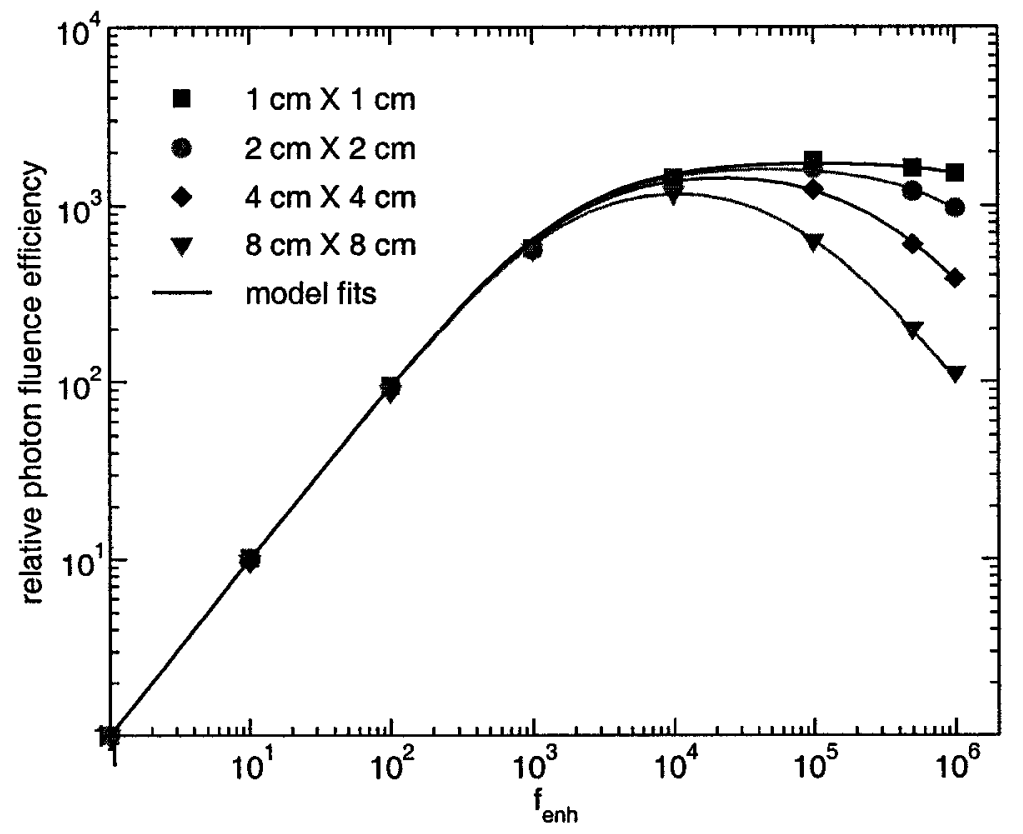

Figure 2.3: The ratio of photon fluence efficiency of a simulation with BCSE over the photon fluence efficiency of the same simulation without BCSE, as a function of the cross section enhancement factor $\left(f_{\text {enh }}\right)$ for the $130 \mathrm{kV} \mathrm{DC} \mathrm{diagnostic} \mathrm{tube}$ described in section 2.4.3. Results are shown for four grids of square scoring zones in a $40 \times 40 \mathrm{~cm}^{2}$ field at $100 \mathrm{~cm}$ SSD. The sum of the variance for all the scoring zones within each grid is used for efficiency calculations. The relative efficiency behavior can be interpreted using Kawrakow's model ${ }^{17,39}$ (solid lines). $f_{\text {enh }}^{\text {opt }}$ varies with the scoring zone size roughly as $1 / \sqrt{A}$.

Figure 2.3 shows the variation of the relative photon fluence efficiency with $f_{\text {enh }}$. It can be seen that the numerical value of the relative efficiency is almost equal to the numerical value of $f_{e n h}$ in the range 1-1000, independent of the scoring zone size. This can be explained as follows: For very small $f_{e n h}$, less than one photon is emitted per incident electron, and so all photons fully contribute to reducing the variance in the scoring zones they fall into. Meanwhile, the CPU time does not increase appreciably because photon transport consumes negligible CPU time compared to electron transport. As $f_{\text {enh }}$ gets relatively larger (1001000), more than one photon can be generated from the same incident electron; however, they on average still fall into different scoring zones and fully contribute 
to reducing the variance in these zones, regardless of the scoring zone size. This means that the effect of cross section enhancement in this range is equivalent to increasing the number of histories without the attendant time penalty; and because efficiency goes linearly with the number of histories, it also does so with $f_{\text {enh }}$ in that range. As $f_{\text {enh }}$ gets much larger $(>1000)$, many more than one photon are generated per incident electron. Such photons start falling in the same scoring zones and they do not contribute as much to reducing the variance because of the relative increase in correlation between them, ${ }^{38}$ yet they still consume the same CPU time to be tracked in the target and thus, according to equation 2.1, the relative efficiency saturates and then drops. The bigger the scoring zone size, the more chance photons generated from the same incident electron fall into a given scoring zone. This causes the peaks of relative photon fluence efficiency for bigger scoring zones to be smaller in magnitude and at smaller $f_{\text {enh }}$ values compared to the peaks for smaller scoring zones.

The efficiency behavior shown in figure 2.3 can also be explained in light of a recent theoretical model derived by Kawrakow. ${ }^{39}$ The model is derived for UBS and DBS to explain the functional form of the relative photon fluence efficiency versus the splitting number, and to predict the optimum splitting number. By replacing $N_{\text {split }}$ in the derivation with $f_{\text {enh }}$, the derivation becomes applicable to the BCSE technique. A practical implementation of the derivation for UBS and DBS has recently been published. ${ }^{17}$ Figure 2.3 shows the results of using that same implementation but for BCSE instead of UBS or DBS by replacing $N_{\text {split }}$ with $f_{e n h}$. The excellent agreement shows that the efficiency behavior when using the BCSE technique can be described theoretically using Kawrakow's model. The model can also be used to predict the optimum $f_{\text {enh }}$ for maximum efficiency gain, which is useful if BCSE is to be used without UBS or DBS.

2.4. RESULTS 


\subsubsection{BCSE in $4 \pi$ geometry (brachytherapy)}

The largest efficiency gains over the current state of the art of the EGSnrc/ BEAMnrc system are expected when BCSE is combined with UBS for $4 \pi$ geometry simulations with low incident charged particle energy. This expectation is because UBS is the only currently available variance reduction technique in the EGSnrc system for $4 \pi$ geometry simulations. The reported UBS efficiency gains are not that large on their own, and so BCSE will boost such gains, particularly at low energies where bremsstrahlung production is very rare.

An example of this situation is the calculation of the three dimensional dose distribution $^{28}$ from the Xoft Axxent ${ }^{\mathrm{TM}}$ source, a miniature brachytherapy electronic X-ray source developed by Xoft. ${ }^{41}$ The source consists of an electron gun in an evacuated tube with a thin coating of target material on a conically shaped anode surface. The source operates between 40 and $50 \mathrm{kV}$ DC. The geometry is modeled using Yegin's general purpose geometry package ${ }^{42}$ for EGSnrc, and the simulation is done using BrachyDose. ${ }^{40}$ Analog simulations of the source inside a $30 \times 30 \times 30 \mathrm{~cm}^{3}$ water phantom show that more than $95 \%$ of the simulation time is spent in generating the photons from the source as opposed to tracking them in the water phantom. This reflects the importance of boosting the efficiency of the process of photon generation. For efficiency calculations, a cube of dimensions $1 \times 1 \times 1 \mathrm{~cm}^{3}$, with the $\mathrm{x}$-ray source at its center, is divided into equal size small voxels with dimensions relevant to clinical practice and to TG-43 dosimetry parameter calculations ${ }^{40}\left(0.3 \times 0.3 \times 0.3 \mathrm{~mm}^{3}, 1.0 \times 1.0 \times 1.0 \mathrm{~mm}^{3}\right.$ and $2.5 \times 2.5 \times 2.5$ $\left.\mathrm{mm}^{3}\right)$. Dose uncertainty is averaged for the voxels making the faces of the $1 \times 1 \times 1$ $\mathrm{cm}^{3}$ cube. Figure 2.4 shows, for an operating voltage of $50 \mathrm{kV} \mathrm{DC}$, the relative dose scoring efficiency for three voxel sizes, both when BCSE is used alone and

\subsection{RESULTS}




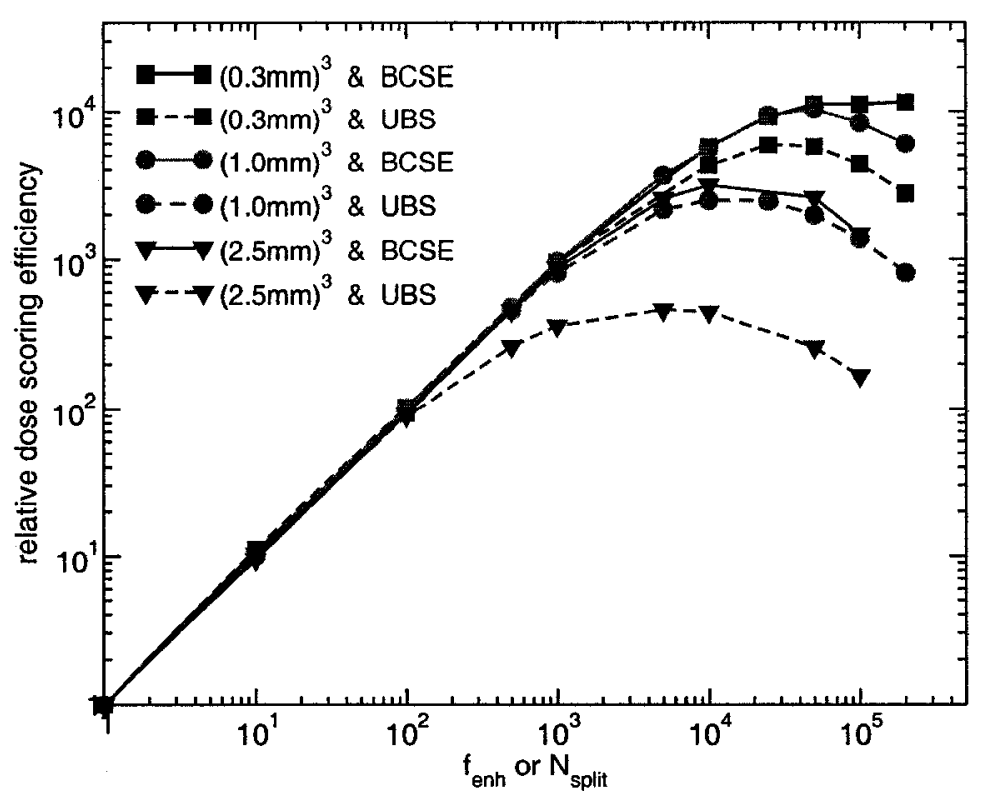

Figure 2.4: The ratio of dose scoring efficiency of a simulation with BCSE or with UBS over the dose scoring efficiency of the same simulation without BCSE or UBS, as a function of the cross section enhancement factor $\left(f_{\text {enh }}\right)$ or the splitting number $\left(N_{\text {split }}\right)$ for the Xoft Axxent ${ }^{\mathrm{TM}} 50 \mathrm{kV}$ DC brachytherapy electronic x-ray source (an example of a $4 \pi$ geometry simulation). BCSE alone outperforms UBS. Further appreciable efficiency gains are achieved when BCSE is combined with UBS for the same simulations (see table 2.1).

when UBS is used alone. Because BCSE creates photons with less correlation than UBS, it allows for a larger $f_{\text {enh }}$ value and produces a higher peak efficiency.

To combine BCSE with UBS, $f_{\text {enh }}$ values of 50, 100, 500, 1000 and 2000 are tested, and each of them is combined with a wide range of $N_{\text {split }}$ values. Table 2.1 summarizes the results for the three voxel sizes investigated. With the parameters as defined in the table caption, the following observations can be made:

- The optimum combination $\left(f_{e n h}^{o p t}, N_{\text {split }}^{\text {opt }}\right)$ yields efficiencies that are up to four orders-of-magnitude larger than those obtained with analog simulations, and up to a full order of magnitude larger than the corresponding peak efficiencies with UBS alone. 
Table 2.1: Efficiency gains when optimally combining BCSE with UBS for the Xoft Axxent ${ }^{T M} 50 \mathrm{kV}$ DC brachytherapy electronic x-ray source (an example of a $4 \pi$ geometry simulation). $f_{\text {enh_oNLY }}^{o p t}$ is the optimum cross section enhancement factor when only BCSE is used. $N_{\text {split_ONLY }}^{\text {opt }}$ is the optimum splitting number when only UBS is used. ( $f_{\text {enh }}^{o p t}, N_{\text {split }}^{\text {opt }}$ ) is the optimum combination of cross section enhancement with splitting. $\varepsilon_{\text {opt }}$ is the ratio of efficiency when optimally combining BCSE with UBS over the efficiency without BCSE or UBS. $R_{B C S E}$ is the ratio of efficiency when optimally combining BCSE with UBS over peak efficiency when $\mathrm{BCSE}$ is used alone. $R_{U}$ is the ratio of efficiency when optimally combining BCSE with UBS over the peak efficiency when UBS is used alone.

\begin{tabular}{|c|r|r|r|r|r|r|}
\hline $\begin{array}{c}\text { side of } \\
\text { cubic voxel } \\
(\mathrm{mm})\end{array}$ & $f_{\text {enh_ONLY }}^{\text {opt }}$ & $N_{\text {split_ONLY }}^{\text {opt }}$ & $\left(f_{\text {enh }}^{\text {opt }}, N_{\text {split }}^{\text {opt }}\right)$ & $\varepsilon_{\text {opt }}$ & $R_{B C S E}$ & $R_{U}$ \\
\hline 0.3 & 200,000 & 25,000 & $(500,200)$ & 13,032 & 1.13 & 2.22 \\
1.0 & 50,000 & 10,000 & $(500,100)$ & 14,750 & 1.42 & 6.43 \\
2.5 & 10,000 & 5,000 & $(1000,50)$ & 4,510 & 1.44 & 9.81 \\
\hline
\end{tabular}

- When BCSE is combined with UBS, $f_{e n h}^{o p t}$ is not very sensitive to voxel size change because $f_{\text {enh }}^{\text {opt }}$ does not get very large.

- $R_{U}$ is much larger than $R_{B C S E}$. This means that most of the variance reduction is achieved through the cross section enhancement rather than through the splitting.

- For a given source energy, $R_{U}$ is larger for bigger scoring voxels because the advantage of cross section enhancement over splitting in creating less correlated photons is more prominent.

\subsubsection{BCSE in directional geometry (diagnostic, mammog- raphy and orthovoltage tubes)}

For directional geometry (as opposed to $4 \pi$ geometry), the largest efficiency gains are expected when BCSE is optimally combined with DBS, with larger gains for lower incident charged particle energies. This expectation is because DBS is the

\subsection{RESULTS}


best splitting option for small solid angle simulations. BCSE will then boost DBS efficiency gains by reducing the correlation between scored particles, especially at lower incident charged particle energies.

To quantify BCSE performance in directional geometry simulations, diagnostic, mammography and orthovoltage tubes are simulated using BEAMnrc. The diagnostic tube specifications are: a $20^{\circ}$ tungsten target in vacuum with copper backing, a beryllium window, aluminum added filtration, lead collimators, and a pair of heavy metal jaws to shape rectangular fields. Simulation parameters are: $20 \times 20$ and $40 \times 40 \mathrm{~cm}^{2}$ fields at $100 \mathrm{~cm}$ SSD and grid scoring zone sizes of $1 \times 1$, $2 \times 2$ and $4 \times 4 \mathrm{~cm}^{2}$. The tube operates at $130 \mathrm{kV} \mathrm{DC}$. For the mammography tube, the differences from the diagnostic one are: a molybdenum target, molybdenum added filtration, an $18 \times 18 \mathrm{~cm}^{2}$ field at $65 \mathrm{~cm} \mathrm{SSD}$ and a grid scoring zone size of $1.5 \times 1.5 \mathrm{~cm}^{2}$. The tube operates at $20 \mathrm{kV} \mathrm{DC}$. The orthovoltage tube specifications and simulation parameters are similar to those of the Siemens Stabilipan2 TH300 unit reported by Verhaegen et al. ${ }^{19}$ This includes: a $24^{\circ}$ tungsten target, aluminum filtration, a close-ended lead-lined applicator with a $3 \mathrm{~mm}$ thick polymethylmethacrylate (PMMA) endplate, a $10 \times 10 \mathrm{~cm}^{2}$ field at $52 \mathrm{~cm}$ SSD and a grid scoring zone size of $1 \times 1 \mathrm{~cm}^{2}$. The tube operates at $230 \mathrm{kV} \mathrm{DC}$. Exact specifications of the tubes simulated are not given here because these tubes are meant to be only representatives of different classes of x-ray tubes.

Simulations with BCSE alone, with UBS alone and with DBS alone are done first, and the corresponding relative photon fluence efficiency curves are plotted. As expected, DBS at its peak outperforms both BCSE and UBS at their peaks by about an order of magnitude, simply because of the directional nature of the DBS algorithm. 
To combine BCSE with UBS or DBS, $f_{\text {enh }}$ values of $10,50,100,200,300$, 400, 500 and 1000 are tested, and each of them is combined with a wide range of $N_{\text {split }}$ values for both UBS and DBS. Table 2.2 summarizes the results for all the investigated tube configurations, source energies, field sizes and grid scoring zone sizes. With the parameters as defined in the table caption, the following observations can be made:

- The optimum combination $\left(f_{\text {enh }}^{o p t}, N_{s p l i t}^{o p t}\right)$ yields efficiencies that are up to five orders-of-magnitude larger than those obtained with analog simulations, and up to 4.8 times larger than the corresponding peak efficiencies with DBS alone.

- For a given tube and source energy, when BCSE is combined with UBS or DBS, $f_{e n h}^{o p t}$ is not very sensitive to field size change or to scoring zone size change.

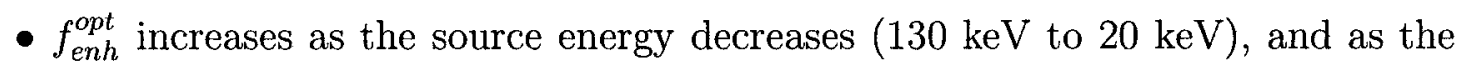
target material changes to a lower- $\mathrm{Z}$ material that produces less bremsstrahlung (tungsten to molybdenum).

- For a given tube, source energy and field size, $f_{\text {enh_onLY }}^{o p t}, N_{\text {split_onLY }}^{\text {opt }}$ and $N_{\text {split }}^{\text {opt }}$ all have a $1 / \sqrt{A}$ dependence on the size of the grid scoring zone $(A)$.

- For a given tube, source energy, field size and grid scoring zone size, $R_{D}$ (the efficiency improvement relative to that of DBS alone) is consistently larger than $R_{U}$ (the efficiency improvement relative to that of UBS alone). This is because, when BCSE is combined with DBS, all the extra photons generated due to the cross section enhancement contribute to the scored quantity (because the ones directed away from the field are not generated except for one fat photon representing them), whereas when BCSE is combined with UBS, all the extra photons are generated and tracked, and only a fraction of them contributes to the scored quantity.

2.4. RESULTS 
Table 2.2: Efficiency gains when optimally combining BCSE with UBS or DBS for the diagnostic, mammography and orthovoltage tubes described in section 2.4.3. Parameters are defined as in the caption of table 2.1, except that $N_{\text {split_onLY }}^{o p t}$ and $\varepsilon_{\text {opt }}$ refer here to either UBS or DBS. In addition, $R_{D}$ is the ratio of efficiency when optimally combining BCSE with DBS over the peak efficiency when DBS is used alone.

\begin{tabular}{|c|c|c|c|c|c|c|}
\hline $\begin{array}{l}\text { field } \\
\text { size } \\
\left(\mathrm{cm}^{2}\right) \\
\end{array}$ & $\begin{array}{c}\text { scoring } \\
\text { zone size } \\
\left(\mathrm{cm}^{2}\right) \\
\end{array}$ & $f_{\text {enh_ONLY }}^{o p t}$ & $N_{\text {split_oNLY }}^{o p t}$ & $\left(f_{e n h}^{o p t}, N_{s p l i t}^{o p t}\right)$ & $\varepsilon_{o p t}$ & $\begin{array}{c}R_{U} \\
\text { or } \\
R_{D} \\
\end{array}$ \\
\hline \multicolumn{7}{|c|}{ mammography tube, $20 \mathrm{kV}$ DC, $65 \mathrm{~cm}$ SSD } \\
\hline $\begin{array}{c}18 \times 18 \\
(\mathrm{UBS}) \\
(\mathrm{DBS})\end{array}$ & $1.5 \times 1.5$ & 500,000 & $\begin{array}{l}50,000 \\
500,000\end{array}$ & $\begin{array}{l}(500,1000) \\
(500,2000)\end{array}$ & $\begin{array}{l}14,512 \\
251,240\end{array}$ & $\begin{array}{l}1.75 \\
4.80\end{array}$ \\
\hline \multicolumn{7}{|c|}{ diagnostic tube, $130 \mathrm{kV} \mathrm{DC,} 100 \mathrm{~cm}$ SSD } \\
\hline $\begin{array}{c}20 \times 20 \\
(\mathrm{UBS})\end{array}$ & $\begin{array}{l}1 \times 1 \\
2 \times 2 \\
4 \times 4\end{array}$ & $\begin{array}{c}100,000 \\
50,000 \\
25,000\end{array}$ & $\begin{array}{l}50,000 \\
25,000 \\
10,000\end{array}$ & $\begin{array}{l}(100,2500) \\
(200,500) \\
(200,200)\end{array}$ & $\begin{array}{l}4,137 \\
3,831 \\
3,179\end{array}$ & $\begin{array}{l}1.19 \\
1.38 \\
1.62\end{array}$ \\
\hline $\begin{array}{c}20 \times 20 \\
(\mathrm{DBS})\end{array}$ & $\begin{array}{l}1 \times 1 \\
2 \times 2 \\
4 \times 4\end{array}$ & & $\begin{array}{l}200,000 \\
100,000 \\
50,000\end{array}$ & $\begin{array}{l}(200,2000) \\
(200,1000) \\
(200,500)\end{array}$ & $\begin{array}{l}84,450 \\
54,874 \\
30,181\end{array}$ & $\begin{array}{l}1.74 \\
2.22 \\
3.37\end{array}$ \\
\hline $\begin{array}{l}40 \times 40 \\
(\mathrm{UBS})\end{array}$ & $\begin{array}{l}1 \times 1 \\
2 \times 2 \\
4 \times 4\end{array}$ & $\begin{array}{c}100,000 \\
50,000 \\
25,000\end{array}$ & $\begin{array}{l}50,000 \\
25,000 \\
10,000\end{array}$ & $\begin{array}{l}(100,2500) \\
(200,500) \\
(200,200)\end{array}$ & $\begin{array}{l}3,816 \\
3,886 \\
3,575\end{array}$ & $\begin{array}{l}1.21 \\
1.42 \\
1.68\end{array}$ \\
\hline $\begin{array}{l}40 \times 40 \\
(\mathrm{DBS})\end{array}$ & $\begin{array}{l}1 \times 1 \\
2 \times 2 \\
4 \times 4\end{array}$ & & $\begin{array}{l}100,000 \\
50,000 \\
25,000\end{array}$ & $\begin{array}{l}(100,2000) \\
(200,1000) \\
(200,500)\end{array}$ & $\begin{array}{l}40,766 \\
35,382 \\
20,222\end{array}$ & $\begin{array}{l}1.37 \\
1.92 \\
2.57\end{array}$ \\
\hline \multicolumn{7}{|c|}{ orthovoltage tube, $230 \mathrm{kV}$ DC, $52 \mathrm{~cm}$ SSD } \\
\hline $\begin{array}{l}10 \times 10 \\
(\mathrm{DBS})\end{array}$ & $1 \times 1$ & 100,000 & 100,000 & $(100,2000)$ & 83,511 & 2.10 \\
\hline
\end{tabular}

2.4. RESULTS 
- For a given tube, source energy and grid scoring zone size, $R_{D}$ is consistently larger for smaller field sizes $\left(20 \times 20 \mathrm{~cm}^{2}\right)$ than it is for larger ones $\left(40 \times 40 \mathrm{~cm}^{2}\right)$. This is because as the field size gets smaller, the optimum splitting number with DBS alone increases as $\sqrt{A}$ but the actual number of photons going towards the field of interest (and thus sampled) decreases roughly as $1 / A$, so the net effect is a reduction in the number of sampled photons by $1 / \sqrt{A}$. This implies that the fractional time spent in tracking the charged particles gets larger, and so the effect of cross section enhancement becomes more obvious.

- For a given tube, source energy and field size, both $R_{U}$ and $R_{D}$ get larger as the grid scoring zone size gets larger (for the same reason discussed before for the voxel size effect in $4 \pi$ geometry).

- For a given tube, source energy and grid scoring zone size, $R_{U}$ and $f_{\text {enh_ONLY }}^{o p t}$ do not change with the field size $\left(20 \times 20\right.$ or $\left.40 \times 40 \mathrm{~cm}^{2}\right)$. This is because, unlike DBS, both UBS and BCSE are non-directional, and all the photons are generated and tracked regardless of the size of the field of interest.

- Both $R_{U}$ and $R_{D}$ increase as the source energy decreases. This is because the bremsstrahlung emission process is rarer, which makes the advantage of combining BCSE with splitting more prominent.

\subsubsection{BCSE with clinical linear accelerators}

For monoenergetic electrons of kinetic energy $6 \mathrm{MeV}$, incident on a $1 \mathrm{~mm}$ tungsten slab followed by a $1.5 \mathrm{~mm}$ copper slab (a typical transmission-type target arrangement in a clinical linear accelerator), our analog Monte Carlo studies show that although about 3.8 bremsstrahlung photons are emitted per incident charged particle, only about $18 \%$ of those bremsstrahlung photons survive target selfattenuation and make it out of the target towards the patient plane. In other

2.4. RESULTS 
words, only $3.8 \times 0.18=0.7$ photons per incident charged particle make it out of the target towards the patient plane. This gives potential for efficiency improvements when BCSE is combined with DBS in the megavoltage range as well.

To quantify BCSE performance in the megavoltage range, $6 \mathrm{MV}$ and $18 \mathrm{MV}$ Varian accelerators with $10 \times 10 \mathrm{~cm}^{2}$ fields at $100 \mathrm{~cm}$ SSD are simulated. Simulation parameters are similar to those used by Sheikh-Bagheri and Rogers. ${ }^{43}$ Two grids within the $10 \times 10 \mathrm{~cm}^{2}$ field are investigated: a grid of equal $1 \times 1 \mathrm{~cm}^{2}$ scoring zones, and a grid of one big $10 \times 10 \mathrm{~cm}^{2}$ scoring zone. To combine BCSE with DBS, $f_{\text {enh }}$ values of $2,5,10,20,30,50$ and 100 are tested, and each of them is combined with a wide range of $N_{\text {split }}$ values for DBS. Table 2.3 summarizes the results for the $6 \mathrm{MV}$ and $18 \mathrm{MV}$ beams. With the parameters as defined in the table caption, it can be seen that even in the megavoltage range with grid scoring zone sizes of clinical relevance, the optimum combination $\left(f_{\text {enh }}^{o p t}, N_{\text {split }}^{\text {opt }}\right)$ yields efficiencies that are up to 800 times larger than those obtained with analog simulations, and up to $40 \%$ larger than the corresponding peak efficiencies with DBS alone. In addition, for the $6 \mathrm{MV}$ beam when the whole $10 \times 10 \mathrm{~cm}^{2}$ field is considered as one big scoring zone, the efficiency gain is a factor of 2.6 over that with optimum DBS alone.

\subsection{Discussion}

When combining BCSE with UBS or DBS, the goal is to find the optimum combi-

nation $\left(f_{\text {enh }}^{o p t}, N_{\text {split }}^{\text {opt }}\right)$ that achieves the maximum efficiency gain for the simulation. Using cross section enhancement creates less correlated photons, but takes a little more CPU time per photon than splitting. Using splitting creates correlated photons, but takes a little less CPU time per photon than cross section enhancement. One would then expect that the optimum combination is to use a cross section

\subsection{DISCUSSION}


Table 2.3: Efficiency gains when optimally combining BCSE with DBS for the $6 \mathrm{MV}$ and $18 \mathrm{MV}$ Varian accelerators ${ }^{43}$ with $10 \times 10 \mathrm{~cm}^{2}$ fields at $100 \mathrm{~cm}$ SSD. Parameters are defined as in the caption of tables 2.1 and 2.2, except that $N_{\text {split_ONLY }}^{\text {opt }}$ and $\varepsilon_{\text {opt }}$ refer here to only DBS.

\begin{tabular}{|c|c|c|c|c|c|c|}
\hline $\begin{array}{c}\text { beam } \\
\text { quality } \\
(\mathrm{MV})\end{array}$ & $\begin{array}{c}\text { scoring } \\
\text { zone size } \\
\left(\mathrm{cm}^{2}\right)\end{array}$ & $f_{\text {enh_ONLY }}^{\text {opt }}$ & $N_{\text {split_oNLY }}^{\text {opt }}$ & $\left(f_{\text {enh }}^{\text {opt }}, N_{\text {split }}^{\text {opt }}\right)$ & $\varepsilon_{\text {opt }}$ & $R_{D}$ \\
\hline 6 & $\begin{array}{c}1 \times 1 \\
10 \times 10\end{array}$ & $\begin{array}{c}4,500 \\
200\end{array}$ & $\begin{array}{c}3,500 \\
250\end{array}$ & $\begin{array}{c}(20,750) \\
(20,50)\end{array}$ & $\begin{array}{c}823 \\
125\end{array}$ & $\begin{array}{c}1.43 \\
2.62\end{array}$ \\
\hline 18 & $1 \times 1$ & 800 & 500 & $(20,100)$ & 83 & 1.21 \\
\hline
\end{tabular}

enhancement factor $f_{\text {enh }}^{O N E}$ that produces exactly one bremsstrahlung photon per incident charged particle (to get as many uncorrelated photons as possible), and then do the rest of the variance reduction through splitting (to save CPU time). Figure 2.1 page 15 can be used to estimate this $f_{e n h}^{O N E}$. For example, for $50 \mathrm{keV}$ monoenergetic electrons incident on a tungsten target (the brachytherapy x-ray source), the probability of emission of one bremsstrahlung photon per incident charged particle is 0.024 and so $f_{\text {enh }}^{O N E}=1 / 0.024 \simeq 42$. Similarly, $f_{\text {enh }}^{O N E} \simeq 250$ for $20 \mathrm{keV}$ electrons incident on a molybdenum target (the mammography tube), $\sim 12$ for $130 \mathrm{keV}$ electrons incident on a tungsten target (the diagnostic tube) and $\sim 9$ for $230 \mathrm{keV}$ electrons incident on a tungsten target (the orthovoltage tube). However, if a cross section enhancement factor of only $f_{e n h}^{O N E}$ is used, selfattenuation in the target material, the tube exit window and the added filtration eliminates many of these uncorrelated bremsstrahlung photons and brings the average number of bremsstrahlung photons exiting the target per incident charged particle to less than unity. In the diagnostic tube described in section 2.4 .3 , only $10 \%$ of the bremsstrahlung photons generated inside the target survive the self-

\subsection{DISCUSSION}


attenuation. This observation suggests that using a cross section enhancement factor $f_{e n h}>f_{e n h}^{O N E}$ to recreate the uncorrelated photons that do not survive selfattenuation will still have an advantage over splitting, as long as the time penalty is not too large.

Figure 2.5 shows an example of the behavior of the efficiency gain while searching for the optimum combination $\left(f_{e n h}^{o p t}, N_{s p l i t}^{\text {opt }}\right)$ for the $6 \mathrm{MV}$ accelerator. Although the scope of the study is the kilovoltage range, the $6 \mathrm{MV}$ accelerator is chosen as an example because in the megavoltage range, efficiency gains are more sensitive to slight variations in $f_{\text {enh }}$ and $N_{\text {split }}$, which serves better in illustrating the behavior of the efficiency gain. It can be seen that many different $\left(f_{\text {enh }}, N_{\text {split }}\right)$ pairs can achieve comparable efficiency gains, although the pair (20,750) gives the maximum. The magnitude of the peak efficiency gains is smaller for very small $f_{\text {enh }}$ ( 5 for example), increase to a maximum $\left(f_{e n h}=20\right)$ and then drop again for larger $f_{\text {enh }}$ (50 for example) when the time penalty is more than the variance reduction that the cross section enhancement gives over the splitting.

Based on the observations above, the following two steps are proposed for optimizing production runs that involve bremsstrahlung targets:

Step 1: The user chooses an optimum cross section enhancement factor depending on the simulation type. Recommended $f_{\text {enh }}^{o p t}$ are summarized in table 2.4. Getting the maximum efficiency gain is not very sensitive to the exact choice of $f_{e n h}^{o p t}$. In addition, $f_{e n h}^{o p t}$ itself is not very sensitive to tube configuration, to scoring zone size, or to voxel size, and so the proposed numbers should suffice and the complementary $N_{\text {split }}^{\text {opt }}$ should pick up any little difference and get the efficiency gain very close to its maximum.

2.5. DISCUSSION 


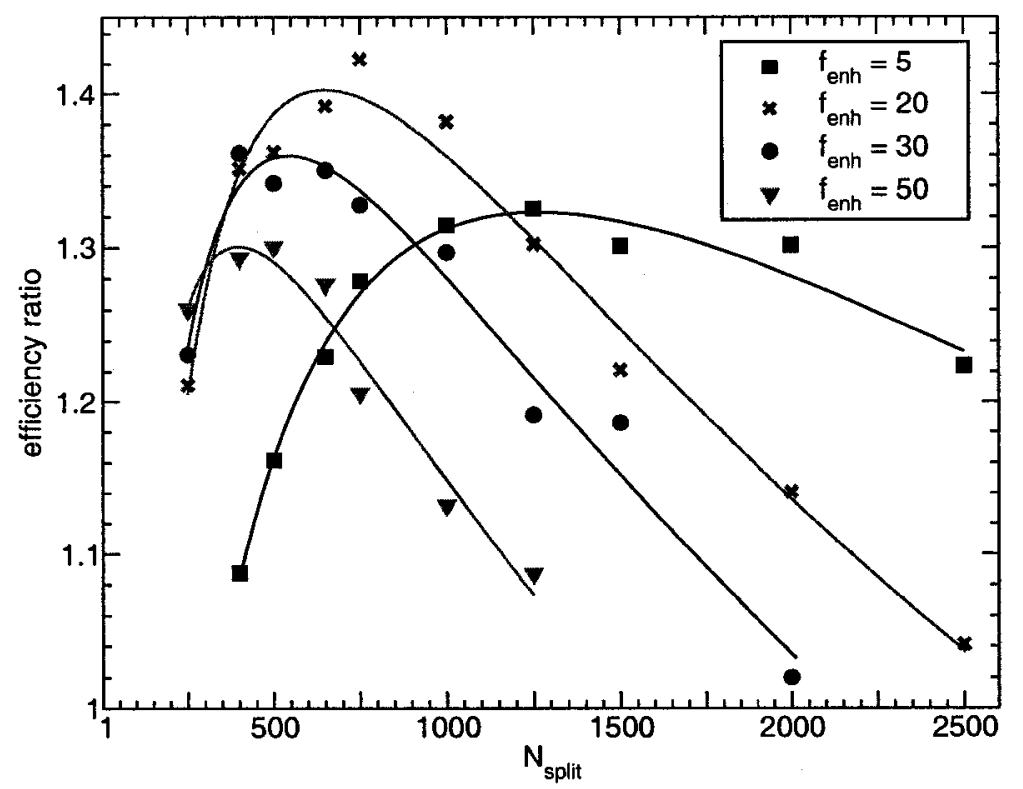

Figure 2.5: The ratio of photon fluence efficiency when BCSE and DBS are combined over the peak efficiency when DBS is used alone, as a function of both the cross section enhancement factor $\left(f_{\text {enh }}\right)$ and the splitting number $\left(N_{\text {split }}\right)$ for the $6 \mathrm{MV}$ Varian accelerator. ${ }^{43}$ A $10 \times 10 \mathrm{~cm}^{2}$ field at $100 \mathrm{~cm}$ SSD is split into equal square scoring zones of $1 \times 1 \mathrm{~cm}^{2}$. Solid lines represent Kawrakow's model.

Step 2: The user determines the optimum $N_{\text {split }}^{\text {opt }}$ (this applies to both UBS and DBS) by applying Kawrakow's model ${ }^{17,39}$ as follows:

- perform a few short runs with $f_{\text {enh }}^{\text {opt }}$ from step 1 combined with a number of $N_{\text {split }}$ values, and calculate the efficiency $\varepsilon_{N_{s p l i t}}$ for each run;

- Fit $N_{\text {split }} / \varepsilon_{N_{\text {split }}}$ versus $\left(N_{\text {split }}-1\right)$ to the following quadratic equation:

$$
\frac{N_{\text {split }}}{\varepsilon_{N_{\text {split }}}}=A_{0}+A_{1}\left(N_{\text {split }}-1\right)+A_{2}\left(N_{\text {split }}-1\right)^{2}
$$

where $A_{i}, i=0,1,2$ are the polynomial coefficients;

- calculate $N_{\text {split }}^{\text {opt }}$ using $N_{\text {split }}^{o p t}=\sqrt{A_{0} / A_{2}}$.

Production runs then use the cross section enhancement factor $f_{\text {enh }}^{o p t}$ from step 1 and the splitting number of $N_{\text {split }}^{\text {opt }}$ from step 2 for maximum simulation efficiency.

\subsection{DISCUSSION}


Table 2.4: Recommended optimum cross section enhancement factors $\left(f_{e n h}^{o p t}\right)$ when combining BCSE with the splitting techniques, for typical situations in diagnostic $x$-ray imaging and in radiotherapy that involve bremsstrahlung targets.

\begin{tabular}{|l|c|c|}
\hline \multicolumn{1}{|c|}{ simulation type } & energy range & recommended $f_{\text {enh }}^{\text {opt }}$ \\
\hline & & \\
$4 \pi$ geometry (brachytherapy) & kilovoltage range & 500 \\
x-ray tubes & mammography range & 500 \\
x-ray tubes & diagnostic range & 200 \\
x-ray tubes & orthovoltage range & 100 \\
clinical linear accelerators & megavoltage range & 20 \\
& & \\
\hline
\end{tabular}

For fluence profiles, when the EGSnrc/BEAMnrc system is optimized as explained above, it usually takes about one minute on a single $3.0 \mathrm{GHz}$ Intel $\AA$ Woodcrest processor to simulate realistic radiotherapy or diagnostic tubes to an average uncertainty of $2 \%$ on photon fluence. Table 2.5 shows the timing results for various situations of clinical interest.

For spectral distributions, when the optimum combination of $f_{e n h}$ and $N_{\text {split }}$ for fluence scoring is used to obtain the spectral distribution, a one minute simulation on the same processor yields reasonably good statistics as shown in figure 2.6. In the figure, large $L$ lines can be seen because of the lack of filtration and because of the relatively larger bin width (which combines close-by L peaks into a few large peaks). Uncertainty on the characteristic peaks is larger than it is on the bremsstrahlung spectrum. This is because all relaxation photons, which are the main contributor to the characteristic peaks, score in very few energy bins, and so the chance of correlated photons falling in the same energy bin is much higher than it is for the bremsstrahlung photons. Longer simulation times $(\sim$ a few minutes) yield better and more uniform statistics.

\subsection{DISCUSSION}


Table 2.5: CPU time, $T(2 \%)$, required to reach an average of $2 \%$ uncertainty on the fluence when an optimized EGSnrc/BEAMnrc system is used to simulate realistic radiotherapy and diagnostic tubes. Simulations are done on a single 3.0 $\mathrm{GHz}$ Intel( $\mathrm{B}$-Woodcrest processor using a g77 compiler.

\begin{tabular}{|l|c|c|c|c|}
\hline \multicolumn{1}{|c|}{ simulation case } & $\begin{array}{c}\text { SSD } \\
(\mathrm{cm})\end{array}$ & $\begin{array}{c}\text { field } \\
\text { size } \\
\left(\mathrm{cm}^{2}\right)\end{array}$ & $\begin{array}{c}\text { scoring } \\
\text { zone size } \\
\left(\mathrm{cm}^{2}\right)\end{array}$ & $\begin{array}{c}T(2 \%) \\
(\text { seconds })\end{array}$ \\
\hline 20 kV DC mammography tube & 65 & $18 \times 18$ & $1.5 \times 1.5$ & 43 \\
$130 \mathrm{kV}$ DC diagnostic tube & 100 & $20 \times 20$ & $1 \times 1$ & 100 \\
& & & $2 \times 2$ & 56 \\
& & & $4 \times 4$ & 21 \\
& 100 & $40 \times 40$ & $1 \times 1$ & 146 \\
& & & $2 \times 2$ & 85 \\
& & & $4 \times 4$ & 22 \\
& & $10 \times 10$ & $1 \times 1$ & 37 \\
$6 \mathrm{MV}$ Varian accelerator & 100 & $10 \times 10$ & $1 \times 1$ & 20 \\
& & & $10 \times 10$ & 1.4 \\
$18 \mathrm{MV}$ Varian accelerator & 100 & $10 \times 10$ & $1 \times 1$ & 56 \\
& & & & \\
\hline
\end{tabular}

This project intends to make the BCSE macros available as part of the standard EGSnrc/BEAMnrc macros in future releases of the system. The user will have the option (through the graphic user interface of the user-code) to use BCSE. If the option is chosen, the user is asked for three simple inputs: the medium to enhance the bremsstrahlung cross section in, the enhancement factor, and whether or not to play Russian Roulette with secondary charged particles. All the details of the BCSE algorithm will be handled internally by the system. 


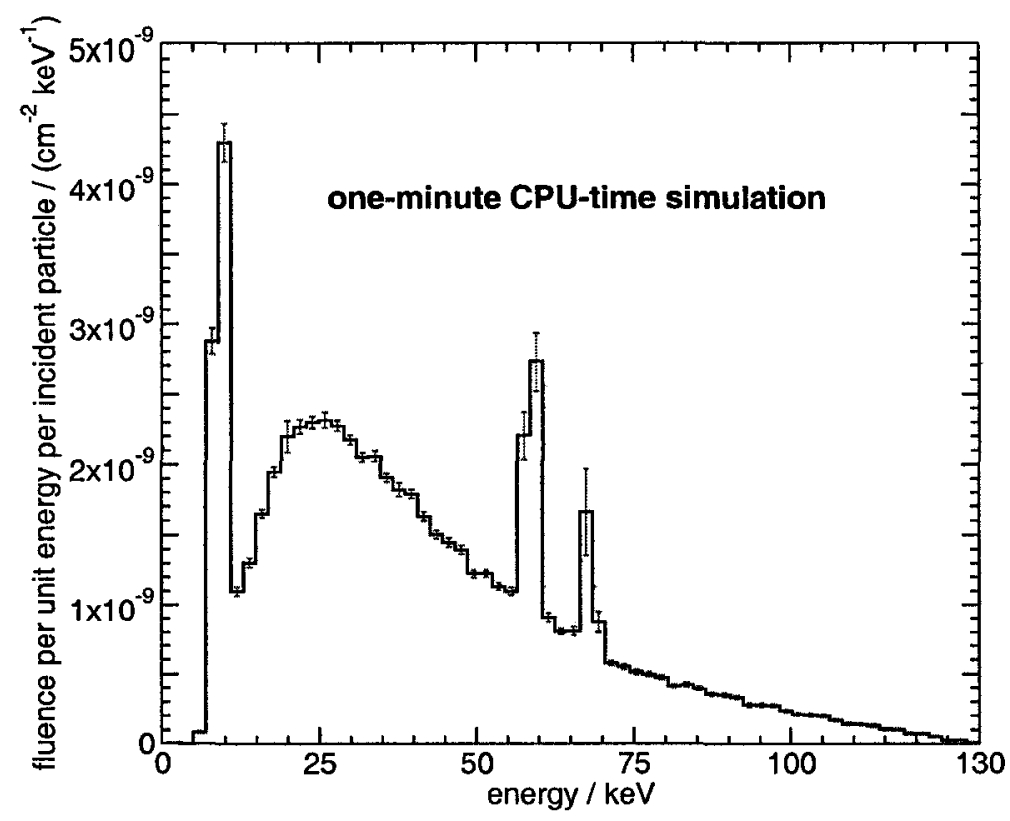

Figure 2.6: A one-minute simulation to obtain the spectral distribution for the unfiltered $130 \mathrm{kV}$ DC diagnostic tube described in section 2.4.3. The graph shows the average spectrum over a $20 \times 20 \mathrm{~cm}^{2}$ field at $100 \mathrm{~cm}$ SSD in $2 \mathrm{keV}$ energy bins. The optimum combination of $f_{e n h}$ and $N_{s p l i t}$ for fluence scoring is used. Simulation is done on a single $3.0 \mathrm{GHz}$ Intel $(\mathrm{B})$-Woodcrest processor using a g77 compiler.

\subsection{Summary}

In this part of the project, the bremsstrahlung cross section enhancement variance reduction technique is implemented and benchmarked in the EGSnrc/BEAMnrc system. Combining BCSE with the existing splitting techniques improves the simulation efficiency both in the kilovoltage and megavoltage range. Efficiency gains can be up to five orders-of-magnitude over those obtained with analog simulations, and up to a full order of magnitude over those without BCSE. This study recommends combining BCSE with the existing variance reduction techniques in EGSnrc/BEAMnrc for both kilovoltage and megavoltage simulations. Optimum cross section enhancement factors are proposed, along with an easy two-step algorithm for simulation optimization. 


\section{Chapter 3}

\section{Benchmarking EGSnrc for}

\section{kilovoltage application}

\subsection{Introduction}

The few published benchmarks of the EGSnrc system in the kilovoltage range are mostly subsets of larger benchmarks that focus more on the megavoltage range $\left({ }^{60} \mathrm{Co}\right.$ and above), or have a split focus at best. In this paragraph, only the kilovoltage parts of these benchmarks are highlighted. The first of such benchmarks came with the original release of the EGSnrc system. ${ }^{7}$ It consists of a monoenergetic beam of electrons normally incident of a semi-infinite water surface. Kawrakow ${ }^{7}$ showed that for 10 and $100 \mathrm{keV}$ electron beams, the fraction of energy reflected, calculated using the EGSnrc condensed history algorithm, is step-size independent, and that it agrees with single scattering calculations at the $0.1 \%$ level. Borg et $a l^{44}$ benchmarked EGSnrc against NRC experimental measurements of ionchamber response to kilovoltage photon beams. They showed agreement at the $0.5 \%$ level for energies 50-250 kVp. The agreement is at the $3 \%$ level for lower energies due to uncertainties in ion chamber impurity levels and geometric details. 
Kawrakow and Rogers ${ }^{45}$ compared EGSnrc calculations of electron backscatter coefficients to experimental data. The study used a limited set of experimental data from the literature, and the comparison was done for only aluminum and gold. Neither the energy spectra nor the angular distribution of backscattered electrons was investigated. Their study showed that including electron spin in EGSnrc simulations is essential to get better agreement with experimental data. Verhaegen ${ }^{46}$ reported good agreement between EGSnrc simulations and parallelplate ion-chamber measurements of interface perturbations in kilovoltage photon beams. Most recently, Mainegra-Hing and Kawrakow ${ }^{17}$ showed agreement at the $2 \%$ level between EGSnrc simulations and NRC experimental measurements of HVL for a DC x-ray tube operating in the range $120-200 \mathrm{kV}$.

In this part of the project, charged particle backscatter is used to benchmark the EGSnrc system. The choice of charged particle backscatter for the benchmark is motivated by many reasons. (1) The literature is lacking such a detailed benchmark. (2) Charged particle backscatter is known to be one of the most stringent tests of any Monte Carlo code that uses the condensed history technique ${ }^{7}$ discussed in section 1.1. (3) There is a plethora of experimental data in the literature for charged particle backscatter in the kilovoltage range (see section 3.3 for references). This is mainly because such data are of great importance to many applied physics fields (particularly scanning electron microscopy and microprobe analysis), and investigators in these fields have been continuously enriching the literature with experimental data. (4) With the advent of hybrid CT/MRI systems ${ }^{47,48}$ accurate characterization of electron backscatter is needed in order to predict the performance of an x-ray tube placed in the magnetic field of an MR scanner. (5) Charged particle backscatter plays an important role in accurately predicting dose perturbations due to high-Z inhomogeneities in tissue. ${ }^{49}$ (6) Ac-

\subsection{INTRODUCTION}


curate characterization of electron backscatter is crucial in predicting the effect of off-focal radiation on the output parameters of x-ray tubes (which is the third component of this project).

When charged particles impinge onto a solid target, they undergo elastic and inelastic processes that cause them to lose energy and divert from their original path to the extent that they may scatter back out of the target. The backscatter probability depends on the cross section of both the elastic and the inelastic processes. A larger elastic scattering cross section (as in high- $Z$ targets) causes more large angle deflections and increases the probability that the charged particle backscatters before it stops. Conversely, a larger inelastic scattering cross section (as in low-Z targets) reduces the backscatter probability because the charged particle will have less chance to undergo enough deflections to backscatter before it stops. Consequently, the fraction of incident charged particles that backscatter, their energy spectra and their angular distribution, all depend on the target material, the incident charged particle energy and the angle of incidence. These aspects of the physics of charged particle backscatter are highlighted throughout this chapter.

\subsection{Definitions}

There is a large variation between different investigators in their definition of the parameters used to characterize the backscatter of charged particles. Because this part of the project compares data from more than one experiment on the same graph, consistent terminology is needed. Figure 3.1 and table 3.1 define the parameters used in this benchmark. These definitions should be used to interpret the graphs in section 3.5. The experimental data taken from the literature are mapped to the terms given in table 3.1 .

3.2. DEFINITIONS 


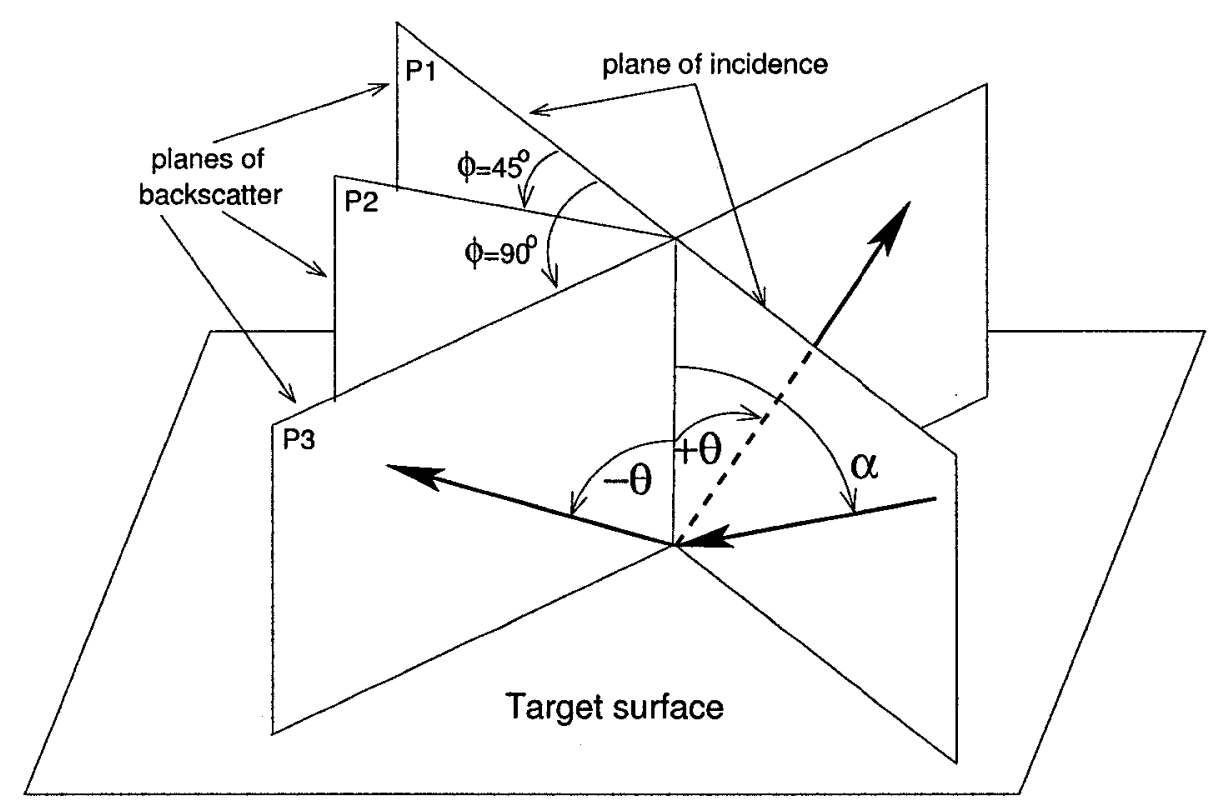

Figure 3.1: Definition of angles and planes used in the benchmark study.

\subsection{Experimental data}

An exhaustive literature search led to the compilation of experimental data from 26 independent experiments performed over a span of 5 decades (1954-2006). Table 3.2 lists the sources of experimental data used in this study. All the experimental datasets are taken from their original sources, except for the dataset with index 2 which is reproduced by Niedrig ${ }^{50}$ from a Ph.D. thesis. When the energy range of an experiment goes beyond the range of interest in this study, only the relevant data are considered. The only mathematical manipulations performed on the experimental data are standard conversions of angles, bins, units and coordinate systems. Examples of such manipulations are the conversion of the integral energy spectra of Darlington ${ }^{51}$ into their differential equivalent, and the conversion of the polar data of Darlinski ${ }^{52}$ into their Cartesian equivalent.

\subsection{EXPERIMENTAL DATA}


Table 3.1: Definition of the parameters used in the benchmark study. The definitions are applicable only to this chapter, not anywhere else in the thesis.

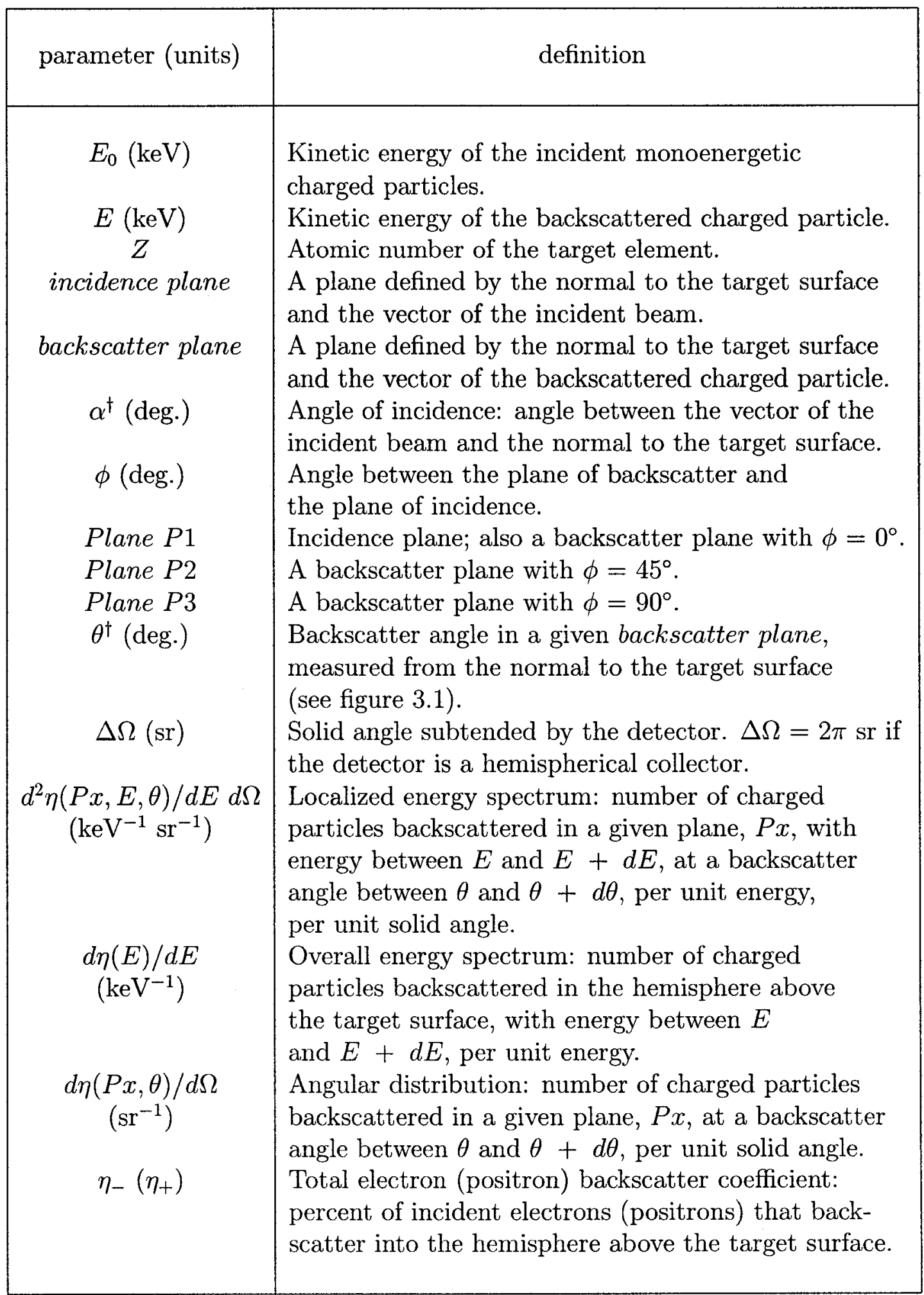

Different experiments report the incidence and backscatter angles with respect to different reference vectors. The reader must map the angles carefully to relate the experimental data reported in this chapter to the sources from which they are taken.

3.3. EXPERIMENTAL DATA 
Table 3.2: Index of experimental data used in the benchmark study.

\begin{tabular}{|c|c|c|c|c|c|c|}
\hline \multirow[t]{2}{*}{ Index ${ }^{a}$} & \multirow{2}{*}{$\begin{array}{c}\text { author(s) } \\
\text { (alphabetically) }\end{array}$} & \multirow[t]{2}{*}{ year } & \multirow{2}{*}{$\begin{array}{c}\text { energy } \\
\text { range } \\
\text { used }^{b} \\
(\mathrm{keV})\end{array}$} & \multicolumn{2}{|c|}{$\begin{array}{l}\text { exp. data } \\
\text { taken from }\end{array}$} & \multirow{2}{*}{$\begin{array}{l}\text { appear in } \\
\text { this chapter } \\
\text { in figure } \\
\text { no. }\end{array}$} \\
\hline & & & & $\begin{array}{c}\text { table } \\
\text { no. }\end{array}$ & $\begin{array}{l}\text { figure } \\
\text { no. }\end{array}$ & \\
\hline $\mathrm{A}$ & Bishop $^{53}$ & 1965 & $5-40$ & 1 & $3,5,7,8$ & $3,4,7,8,9,15$ \\
\hline $\mathrm{B}$ & Coleman et $a l^{54}$ & 1992 & $5-50$ & 1,2 & - & 14,15 \\
\hline $\mathrm{C}$ & Coslett \& Thomas ${ }^{55}$ & 1965 & $10-30$ & 1 & 1,2 & 3,13 \\
\hline $\mathrm{D}$ & Darlington $^{51}$ & 1975 & $10-30$ & 3 & - & 6,8 \\
\hline $\mathrm{E}$ & Darlinski $^{52}$ & 1981 & 30 & - & 2,3 & $9,10,11$ \\
\hline G & Dreshcer et al ${ }^{d, 56}$ & 1970 & $10-100$ & 1 & 2 & $3,4,5$ \\
\hline $\mathrm{H}$ & Fitting \& Technow ${ }^{57}$ & 1983 & 10 & - & 2 & Sec. 3.5.1 \\
\hline $\mathrm{J}$ & Gérard et $a l^{58}$ & 1995 & 30 & - & 19,21 & 6,9 \\
\hline K & Heinrich $^{59}$ & 1965 & $10-49$ & 3 & - & $3,4,15$ \\
\hline $\mathrm{L}$ & Hunger \& Küchler ${ }^{60}$ & 1979 & $5-41$ & 1 & - & $3,4,15$ \\
\hline M & Kanter ${ }^{d, 61}$ & 1957 & $50 \& 70$ & - & $5,9,10$ & 7,12 \\
\hline $\mathrm{N}$ & Kanter ${ }^{62}$ & 1964 & $10 \& 15$ & - & 3 & 13 \\
\hline $\mathrm{P}$ & Kulenkampff \& Rüttiger ${ }^{d, 63}$ & 1954 & 30 & 2 & 8 & 8 \\
\hline $\mathrm{R}$ & Kulenkampff \& Spyra ${ }^{d, 64}$ & 1954 & $20-40$ & 1,2 & 5 & $3,4,6,8$ \\
\hline $\mathrm{S}$ & Mäkinen et $a l^{65}$ & 1992 & $5-30$ & - & 2 & 14,15 \\
\hline $\mathrm{T}$ & Martin et $a l^{66}$ & 2006 & $40-124$ & - & 3 & 3 \\
\hline $\mathrm{U}$ & Massoumi et $a l^{67}$ & 1991 & 35 & 1 & - & 14,15 \\
\hline W & Massoumi et $a l^{68}$ & 1993 & $5-35$ & - & $5,7,8,9$ & $3,4,15,16$ \\
\hline $\mathrm{X}$ & Matsukawa et $a l^{69}$ & 1974 & 20 & - & 3,4 & 6 \\
\hline $\mathrm{Z}$ & Neubert \& Rogaschewski ${ }^{70}$ & 1980 & $15-60$ & 1,2 & - & $3,4,5,15$ \\
\hline 2 & Niedrig $^{50}$ & 1982 & $20 \& 30$ & - & 25 & 13 \\
\hline 3 & Niedrig \& Sieber ${ }^{d, 71}$ & 1971 & $20-53$ & - & 2 & 13 \\
\hline 4 & Rau et $a l^{72}$ & 2002 & 20 & - & 2 & 13 \\
\hline 5 & Soum et $a l^{e, 73}$ & 1984 & $40 \& 90$ & - & 7 & 3 \\
\hline 7 & Weinryb \& Philibert ${ }^{e, 74}$ & 1964 & $4.5-30$ & 1 & - & 3,4 \\
\hline 8 & Wittry $^{75}$ & 1965 & $5 \& 30$ & 1 & - & 3,4 \\
\hline
\end{tabular}

${ }^{a}$ Letters and numbers that may be confusing in graphs are skipped.

${ }^{b}$ not necessarily the full energy range covered in the experiment.

${ }^{c}$ If the source reports the experimental data more than once or in different forms, only the exact figure or table the data is taken from is mentioned.

${ }^{d}$ article in German.

${ }^{e}$ article in French. 
When experimental datasets are not available in tabular form, they are digitized electronically from their graphs. In section 3.5, indices from table 3.2 are used instead of symbols, both in the graphs and in the captions, in order to avoid graph clutter and text redundancy. Additional notes on specific experiments are mentioned in the captions whenever needed.

Although measurements of charged particle backscatter are conceptually simple (viz. measuring the current due to the incident beam and the current due to the backscattered beam for a given setup), they are very challenging in practice, and they are fraught with sources of uncertainty. Knowledge of these uncertainties is essential for insightful comparison between the results of EGSnrc simulation and such experimental data. A careful examination of the experiments considered in this study indicates that the uncertainties can tentatively be classified into 6 groups, and they are summarized in the following 6 paragraphs. The reader is referred to the references in table 3.2 for detailed discussion of the different experiments and their associated uncertainties.

The first group includes uncertainties due to low energy electrons. When incident charged particles interact with the target material, a large number of very low energy electrons is produced due to various atomic relaxation processes (by convention in the field of scanning electron microscopy, very low energy electrons are those with energy $<50 \mathrm{eV}$ ). Such electrons can enter the charged particle collector and lead to over-estimation of the backscatter coefficient. Experiments avoid this by either using negatively-polarized grids (called retarding grids) at $-50 \mathrm{eV}$ to return these low energy electrons to the target, or by positively biasing the target itself to prevent these low energy electrons from leaving it, or both. A careful application of a retarding grid can largely control (but not eliminate) the problem of low energy electrons. However, a grid correction is needed to account

\subsection{EXPERIMENTAL DATA}


for backscattered electrons intercepted by the grid, and there are non-negligible uncertainties associated with this grid correction. ${ }^{53}$ In addition, small differences in the experimental setup or operational parameters can have a considerable effect on the low energy electron currents. ${ }^{59}$

The second group includes voltage-bias uncertainties. Positively-charged targets attract the backscattered electrons and other electrons produced from different parts of the experimental setup to impinge or re-impinge onto the target. Also, depending on the geometry and voltage bias distribution, backscattered charged particles can hit different parts of the experimental setup and generate multiple lower energy electrons that can alter the results if detected. ${ }^{59}$ Small changes in the bias can also cause systematic variations in the incident beam intensity. ${ }^{65}$

The third group includes target uncertainties. Despite cleaning, polishing and operating at low pressure, surface contamination is always an issue to varying extents; ${ }^{75}$ it leads to under-estimation of the backscatter fraction because surface contaminants are generally lower-Z materials that have a backscatter coefficient lower than that of the target material (as will be shown in section 3.5.1). Surface contamination is more important at lower energies and at grazing incidence ${ }^{54}$ because at such energies and angles the effective perpendicular penetration of the charged particle decreases, and hence the effect of the contaminant layer increases. For backscatter from thin film targets (as opposed to bulk targets), uncertainty in the thickness of the film target can be up to $10 \%{ }^{72}$

The fourth group includes geometric uncertainties. Small incident beam movements can cause fluctuations in the detector response. ${ }^{54}$ Setup or operational constraints can lead to deviations of up to $5^{\circ}$ from normal incidence while still calling it normal incidence. ${ }^{54,65}$ The non-zero diameter of the incident beam

\subsection{EXPERIMENTAL DATA}


can become an issue at grazing incidence angles because it becomes harder to guarantee that all charged particles hit the target. ${ }^{68}$ Uncertainties in alignment of detectors, angles and planes can add $\sim 2 \%$ to the overall uncertainty. ${ }^{66}$ In general, geometry effects can be large enough that certain experiments apply a dedicated geometry correction factor. ${ }^{60}$

The fifth group includes detector uncertainties. The lower part of the energy spectrum of the backscattered charged particles is noisier and it has poorer resolution. ${ }^{53,68}$ Energy calibration and the exact determination of the point of zero energy loss can also be an issue. ${ }^{53}$ Detector pulse pileup can occur when more than one backscattered electron reaches the detector within the detector resolving time, and the two electrons erroneously count as one charged particle with kinetic energy equal to the sum of the two. ${ }^{68}$ The accuracy of the angular distribution of backscattered charged particles can be affected by the exact positioning of the detector in high-gradient regions; uncertainty can be up to $12 \%$ for extreme angular distribution gradients. ${ }^{52}$ Electrons backscattered from the target may also backscatter from the face of the detector when they reach it; ${ }^{68}$ this effect may cancel out because it affects the measurement of both the incident and the backscattered current (assuming the effect is energy independent). At grazing backscatter angles, and because of the finite solid angle subtended by the detector, a portion of the detector may be masked by the target itself; 67 this leads to underestimation of the backscatter coefficient. There are also uncertainties associated with extracting the true energy spectra from the overall detector response. ${ }^{66,68}$ Finally, lower energy backscattered electrons may completely stop in the detector entrance window ${ }^{58}$ this can lead to under-estimation of the backscatter coefficient, and it may not be possible to account for it using the detector response function.

\subsection{EXPERIMENTAL DATA}


The sixth and last group includes data analysis uncertainties. Some experiments use hemispherical collecting electrodes to determine the overall backscatter coefficient. For various reasons (including experimental convenience), other experiments take measurements at single or multiple fixed angles to determine the energy spectra and/or the angular distribution, then certain mathematical and numerical procedures are applied to estimate the overall backscatter coefficient. Such procedures have uncertainties associated with them. For example, Massoumi et $a l^{68}$ estimated the uncertainty in their experiment due to data analysis to be about $10 \%$ for $\mathrm{Z}>20$, and $>10 \%$ for $\mathrm{Z}<20$.

Some of the uncertainties discussed above are more challenging than others, and some experiments are more careful than others in reducing these uncertainties. The most recent experiment included in the benchmark ${ }^{66}$ uses two different measurement techniques - current integration and silicon detector measurements. The reported reproducibilities are within 5 and $7 \%$, respectively. With the addition of other uncertainties, the reported overall uncertainties are 9 and $12 \%$, respectively. In general for all the experiments that measure charged particle backscatter, the overall uncertainty is higher for lower incident energies (due to the effects of low energy electron currents, sample contamination, detector resolution, detector entrance window, ... etc), and for lower-Z targets (due to smaller backscatter yield). The uncertainties discussed above can explain the variation between different experiments measuring the same parameter. They can also help in evaluating the quality of agreement between EGSnrc results and the experimental data.

\subsection{EGSnrc simulation}

Throughout this benchmark study, the EGSnrc simulations employ the most accurate low energy physics and the most accurate cross sections that EGSnrc offers for

\subsection{EGSNRC SIMULATION}


charged particles and for photons as discussed in section 1.1. Simulations are done using DOSRZnrc, an EGSnrc user-code, but many other user-codes could have been used instead. Simulations are for pencil beams of monoenergetic charged particles with energy between 5 and $140 \mathrm{keV}$, incident on 20 -cm thick targets with $\mathrm{Z}$ between 4 and 92 . The target thickness of $20 \mathrm{~cm}$ is considered infinite because it is much larger than the range of the incident charged particles. Both normal and oblique incidence are simulated. Tallying the backscattered charged particles is done using a macro newly added to DOSRZnrc as part of this study. Details of the macro features can be found in Ali and Rogers. ${ }^{3}$ The macro reports the following for the backscattered charged particles: the total backscatter coefficient, the overall energy spectrum (i.e. $\Delta \Omega=2 \pi \mathrm{sr}$ ), the localized energy spectra for the backscatter angles chosen by the user, and the angular distribution for the backscatter planes chosen by the user.

For this study, three backscatter planes, P1, P2 and P3, are defined (see table 3.1). In each plane, 59 scoring regions (representing detectors) are distributed uniformly on the circumference of a semi-circle of radius $5 \mathrm{~cm}$ (a typical radius for placing detectors in backscatter experiments), with the face of the scoring regions perpendicular to the plane of backscatter. For the energy spectra, 120 energy bins are used, which implies that the width of each energy bin is $E_{0} / 120$. All simulations are analog (i.e. no variance reduction techniques). However, very good statistics on the scored parameters can be obtained in a reasonable amount of time ( $\sim$ minutes to hours) because backscatter is a relatively abundant event. Simulation times becomes longer $(\sim$ a few days) when the parameter of interest is the energy spectrum recorded in a very small scoring region, with higher energy beams incident on higher-Z targets.

\subsection{EGSNRC SIMULATION}


When BEAMnrc is used instead of DOSRZnrc to estimate the backscatter parameters, simulation results are found to be sensitive to the choice of an internal parameter in BEAMnrc called the boundary tolerance. This parameter is used in BEAMnrc to avoid round-off errors by artificially moving the boundaries between different regions in the geometry by a small distance $\$ B D Y \_$TOL. The default value of $\$ B D Y \_$TOL is $10^{-5} \mathrm{~cm}$, and the user has the option to adjust it. As mentioned in section 1.1, the BEAMnrc system has been mainly designed for and used in the simulation of clinical linear accelerators. This very small boundary adjustment has absolutely no effect in the megavoltage range and in the upper kilovoltage range. However, at low energies, the charged particle step sizes are in the same order of magnitude as the default value of $\$ B D Y \_T O L$, hence the default value is too large at such energies.

Figure 3.2 shows the variation versus $\$ B D Y \_$TOL of the percent deviation of BEAMnrc results from the correct value of the total backscatter coefficient for low- and high- $\mathrm{Z}$ targets at three different energy ranges. It can be seen that the default value is adequate at megavoltage energies regardless of the atomic number of the target. At about $100 \mathrm{keV}$, the default value can cause deviations up to $5 \%$ for high-Z targets. In the mammography range with a high-Z target, the deviation can be up to $50 \%$. In addition, the default value of $\$ B D Y$ TOL causes a slight shift towards lower values in the energy spectrum of the backscattered electrons. When $\$ B D Y$ TOL is reduced to $5 \times 10^{-7} \mathrm{~cm}$, deviations in the total backscatter coefficient drastically diminish.

The effect of the value of \$BDY.TOL is not as dramatic when the focus of the simulation is on parameters other than charged particle backscatter, but it still exists. For example, when simulating a $20 \mathrm{keV}$ monoenergetic beam of electrons normally incident on a semi-infinite tungsten target, larger values of $\$ B D Y$ BOL

\subsection{EGSNRC SIMULATION}


cause a slight but noticeable hardening of the photon energy spectrum at the patient plane $(\mathrm{SSD}=100 \mathrm{~cm})$. Therefore, this study recommends using a value of $\$$ BDY_TOL $=5 \times 10^{-7} \mathrm{~cm}$ for all kilovoltage medical physics applications that use component modules in BEAMnrc which use the $\$ B D Y$ TOL parameter, regardless of the focus of the application. For the backscatter calculations under consideration in this part of the project, the value of $\$ B D Y_{-}$TOL $=5 \times 10^{-7} \mathrm{~cm}$ limits the maximum deviation to less than $2 \%$ for the extreme case of mammography situations. Values of $\$ B D Y$ TOL lower than $5 \times 10^{-7} \mathrm{~cm}$ require BEAMnrc to run fully in double precision.

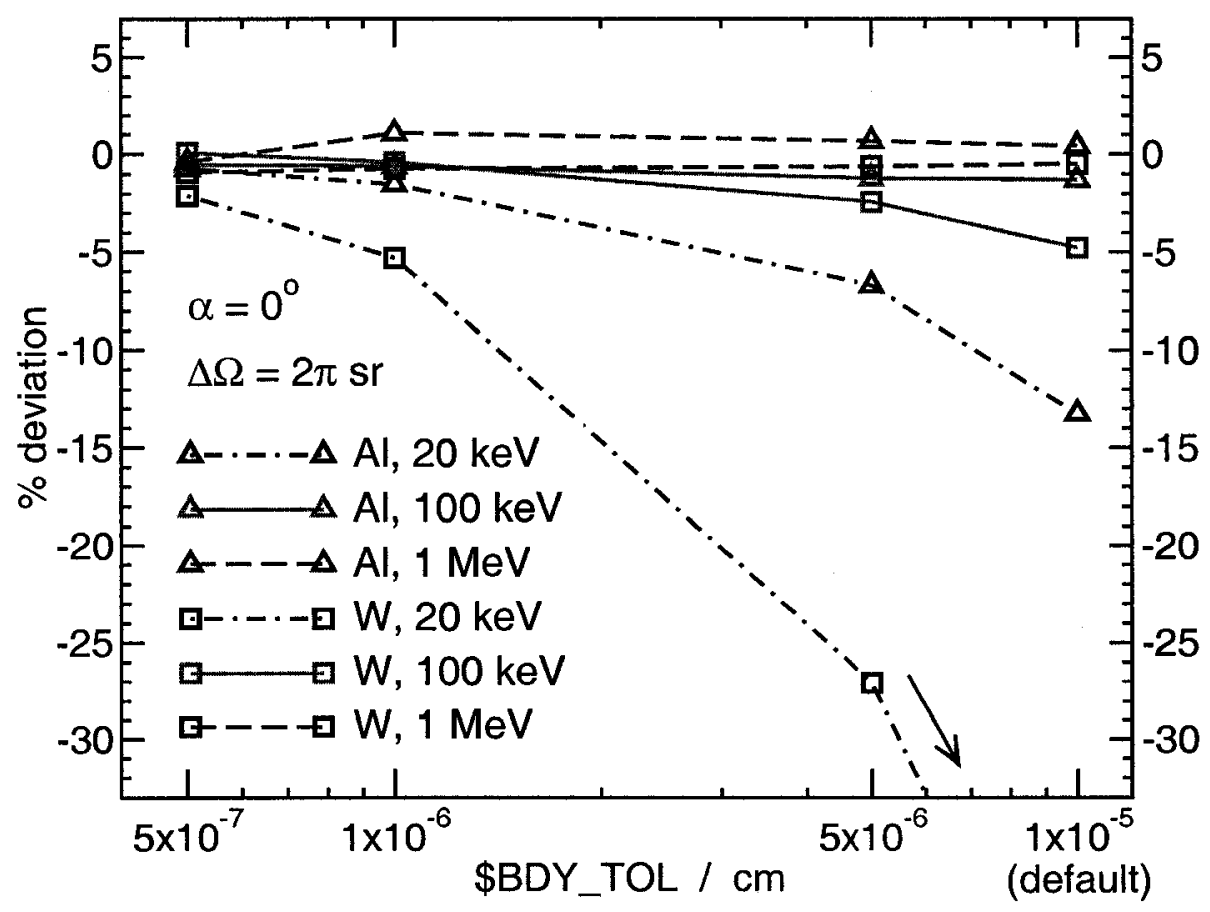

Figure 3.2: Effect of the boundary tolerance parameter (\$BDY_TOL) on the accuracy of backscatter coefficient calculation in BEAMnrc for different target materials at different energies. The correct value is obtained using the DOSRZnrc user-code, which does not have the boundary tolerance issue. The arrow goes to $-50 \%$ at the default value of $\$ B D Y_{-}$TOL (i.e., $10^{-5} \mathrm{~cm}$ ) for $E_{0}=20 \mathrm{keV}$ and a tungsten target. Data points are connected by line segments. The scale of the abscissa is logarithmic. 


\subsection{Results}

This section presents comparisons between EGSnrc simulation results and experimental data taken from the sources in table 3.2. For the figures containing energy and angular distribution curves, the highest peak in the EGSnrc results and the highest peak in the experimental data are both scaled to unity, unless otherwise stated. Other data in the figure are scaled by the same factor, which means that the relative shapes and areas under different curves in a given figure are preserved. Error bars on the simulation results are too small to show. No error bars are shown on the experimental data to avoid clutter of the already crowded graphs, and also because most experiments describe an overall uncertainty associated with their measurements, but they do not give detailed error bars. The reader is referred to section 3.3 , and to the references listed in table 3.2 for more on the uncertainties of the experimental measurements.

\subsubsection{Total backscatter coefficient}

Figure 3.3 compares EGSnrc results versus experimental data for the variation of the total electron backscatter coefficient, $\eta_{-}$, with the incident electron energy, $E_{0}$, for $\alpha=0^{\circ}$. Low $\mathrm{Z}$ targets exhibit a slight decrease in $\eta_{-}$as $E_{0}$ increases, whereas high-Z targets exhibit an opposite trend. EGSnrc simulations replicate this slight energy dependence very well. The discrepancy between some experimental data and the EGSnrc simulations for very-low energy electrons incident on low-Z targets can be attributed to the uncertainties summarized in the last paragraph of section 3.3. Tungsten and molybdenum are the two most commonly-used elements in $\mathrm{x}-$ ray tube targets; the value of $\eta_{-}$for tungsten at diagnostic energies is $\sim 50 \%$, and for molybdenum at mammography energies is $\sim 38 \%$.

3.5. RESULTS 


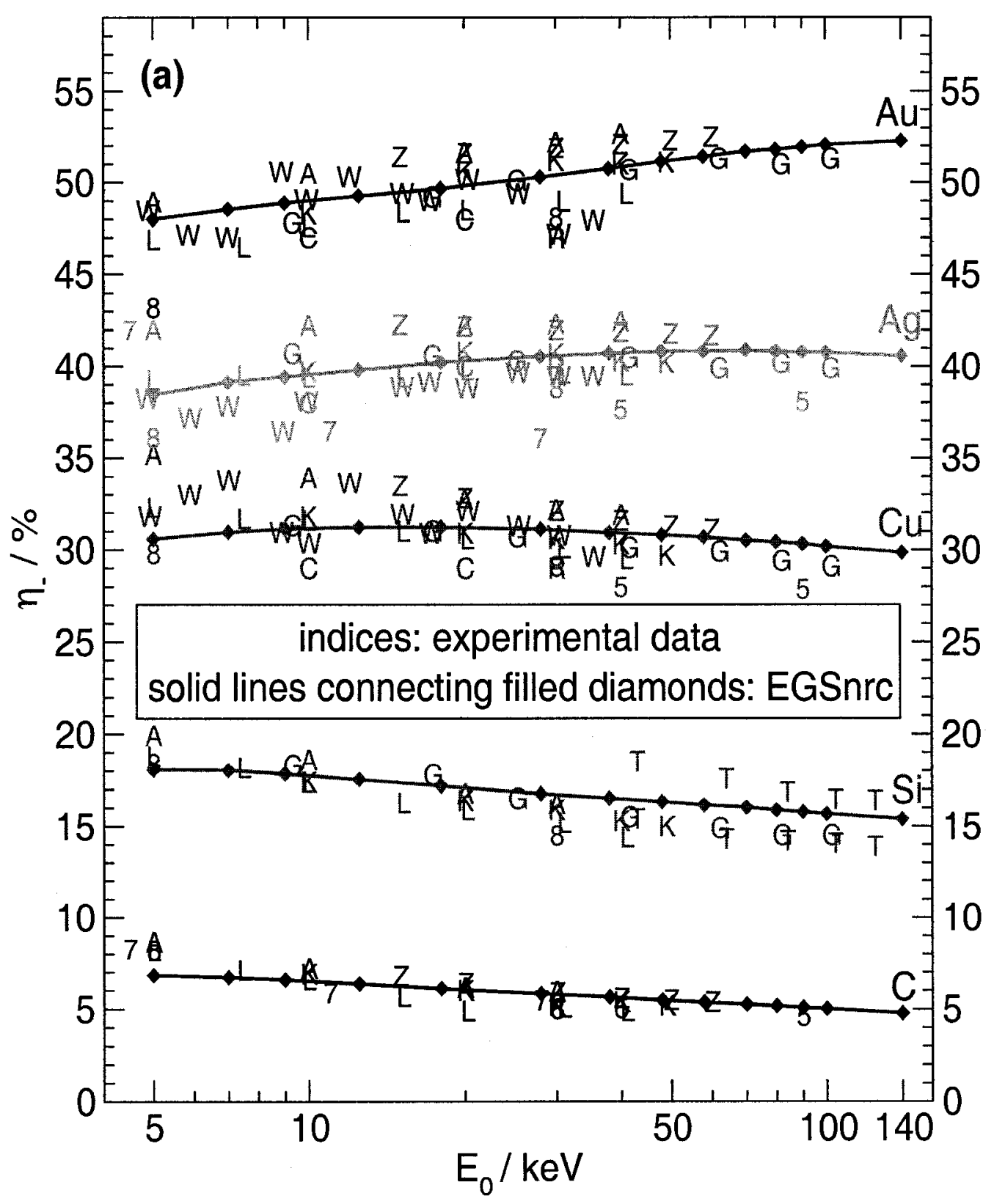

3.5. RESULTS 


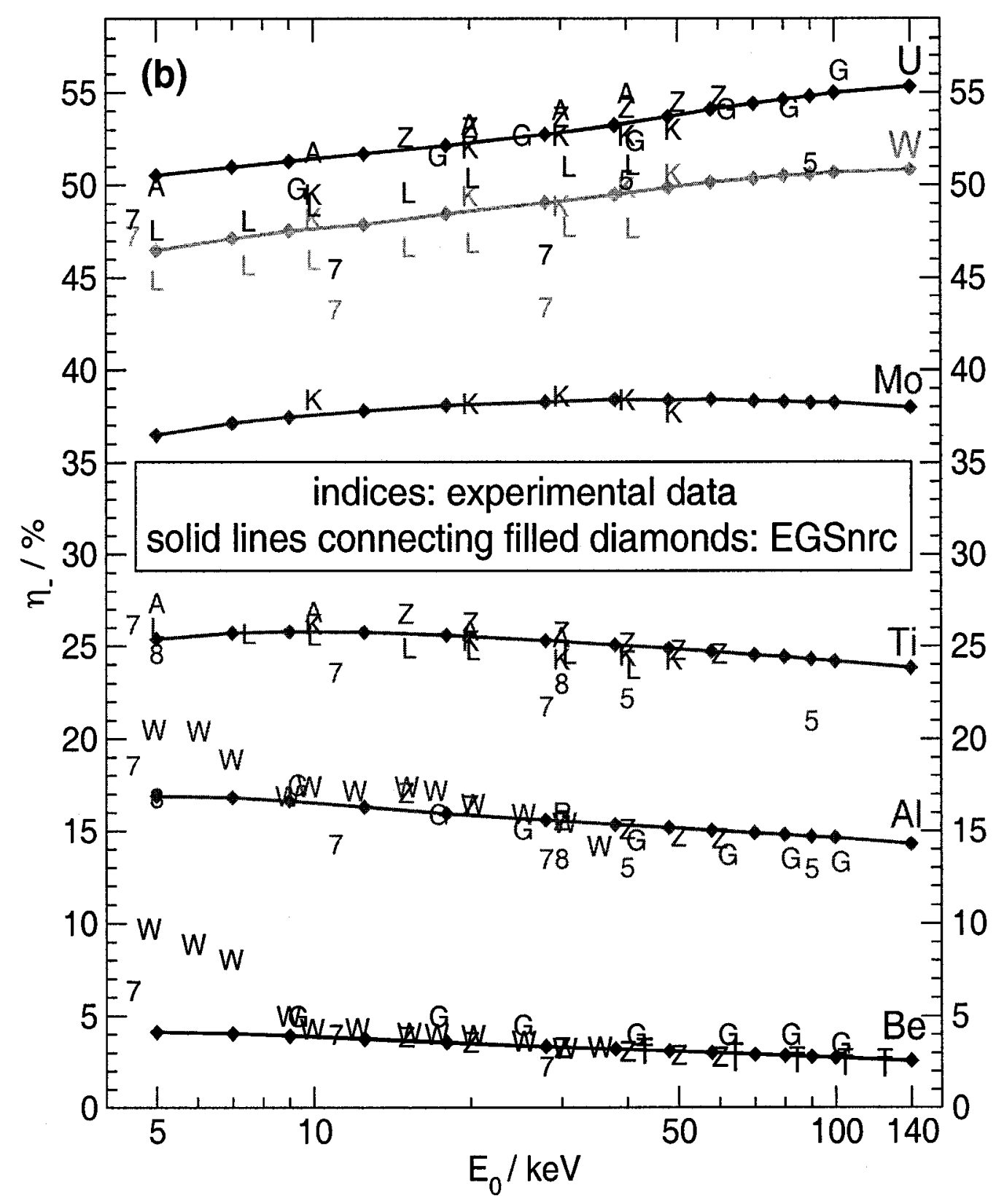

Figure 3.3: EGSnrc results versus experimental data for the variation of the total electron backscatter coefficient, $\eta_{-}$, with the incident electron energy, $E_{0}$, for $\alpha=0$ (normal incidence) and $\Delta \Omega=2 \pi \mathrm{sr}$ (hemispherical collector). EGSnrc simulations (filled diamonds) are done at slightly different energies from the experiments (e.g. 38 instead of $40 \mathrm{keV}$ ) for graph clarity. Simulation results are connected by solid line segments to aid the eye. Experimental data are taken from experiments with indices $A, C, G, K, L, R, T, W, Z, 5,7$ and 8 (see table 3.2 page 44 for the indexing). In panel a for experiment $A$, data for platinum $(Z=78)$ are used for gold $(Z=79)$. Experiment $R$ has only one data point at $30 \mathrm{keV}$ for $\mathrm{Al}, \mathrm{Cu}, \mathrm{Ag}$ and $\mathrm{Au}$. Experiment $\mathrm{T}$ used two different measurement techniques, so there are two values at each energy for both $\mathrm{Be}$ and $\mathrm{Si}$. The scale of the abscissa is logarithmic.

\subsection{RESULTS}


Figure 3.4 compares EGSnrc results versus experimental data for the variation of $\eta_{-}$with the target materials, Z. The value of $\eta_{-}$shows a smooth and monotonic increase with $\mathrm{Z}$ (from $\sim 3 \%$ up to $\sim 50 \%$ for $E_{0}=30 \mathrm{keV}$ ). EGSnrc simulations replicate this monotonic behavior very well. The elastic scattering cross section varies as $Z^{2}$, whereas the inelastic scattering cross section (and hence the stopping power) varies roughly $\operatorname{as}^{54} \mathrm{Z}$. This implies that the elastic scattering increases faster than the inelastic scattering as $\mathrm{Z}$ increases, which leads to more large angle deflections and increases $\eta_{\text {_ }}$ as $\mathrm{Z}$ increases. The experimental data from experiment with index 7 by Weinryb and Philibert ${ }^{74}$ are systematically lower than the rest of the experimental and simulation data. Bishop ${ }^{53}$ explains that this may be because of the systematic errors introduced by the retarding grids used, and by the scattering of electrons back onto the target.

Figure 3.5 compares EGSnrc results versus experimental data for the variation of $\eta_{-}$with the angle of incidence, $\alpha$, for different materials in two different experiments performed at two different energies. The value of $\eta_{-}$monotonically increases with $\alpha$, and the increase becomes more significant as grazing angles are approached. EGSnrc simulations match the two experiments very well, except at near-grazing incidence on high-Z targets. Similar agreement (not shown to avoid redundancy) is obtained between EGSnrc simulations and the experimental data of Fitting and Technow ${ }^{57}$ for $E_{0}=10 \mathrm{keV}$. As $\alpha$ increases, the portion of the forward-peaked differential elastic scattering distribution that falls in the backscatter hemisphere increases, hence more electrons can backscatter out of the target and $\eta_{-}$increases. Also the rate of increase of $\eta_{-}$increases as $\mathrm{Z}$ decreases. This makes the variation of $\eta_{\text {_ }}$ with $\mathrm{Z}$ more dramatic at near-normal incidence compared to near-grazing incidence. The small discrepancy between the EGSnrc simulation results and a few of the experimental data points at very large $\alpha$ can

\subsection{RESULTS}




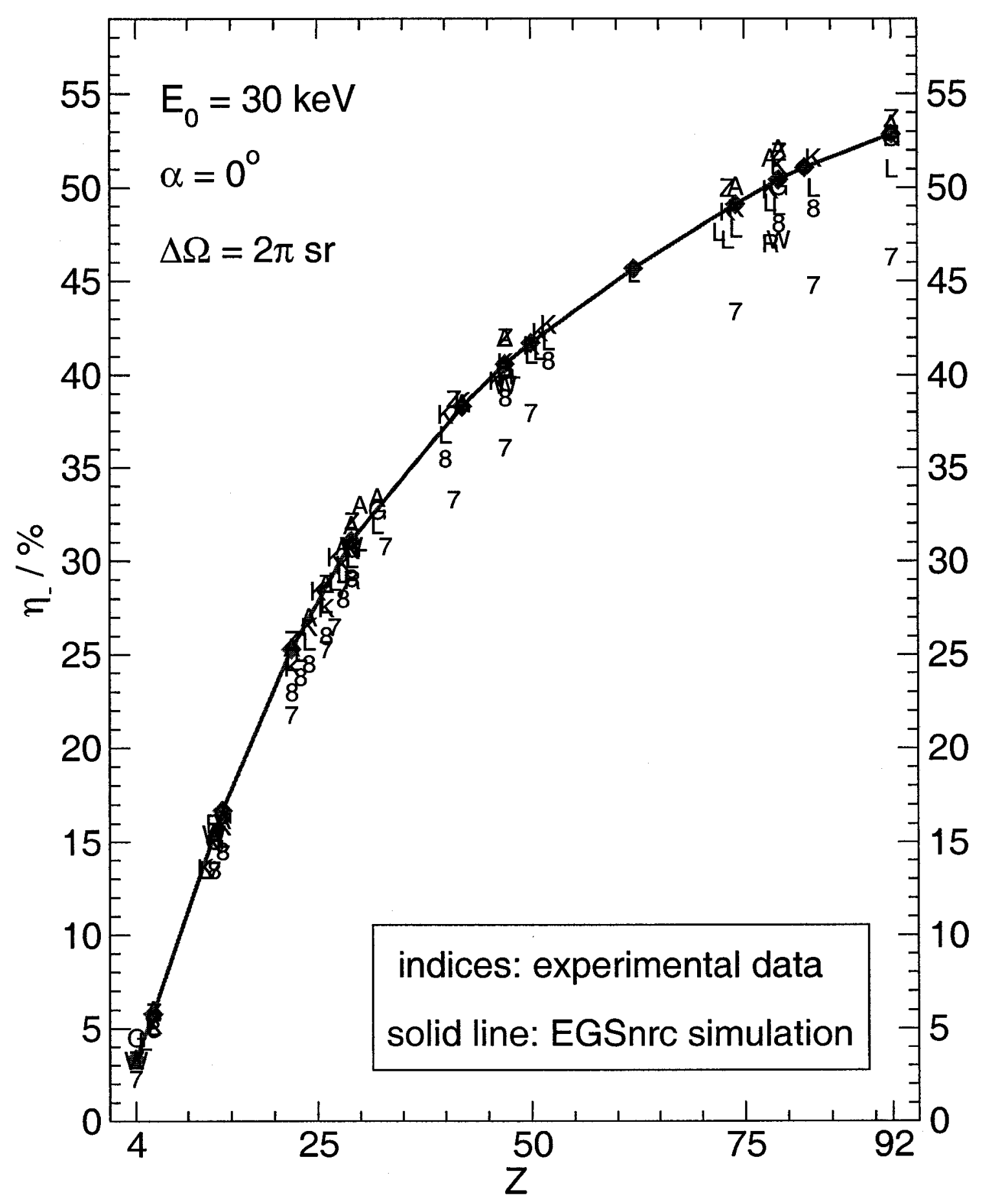

Figure 3.4: EGSnrc results versus experimental data for the variation of the total electron backscatter coefficient, $\eta_{-}$, with the atomic number of the target material, Z. EGSnrc results (filled diamonds) are connected by solid line segments to aid the eye. Experimental data are taken from experiments with indices $A, G, K, L, R, W$, $Z, 7$ and $8 . E_{0}$ in experiments $G, L$ and 7 is not exactly $30 \mathrm{keV}(25.2,31.0$ and $28.0 \mathrm{keV}$, respectively).

3.5. RESULTS 

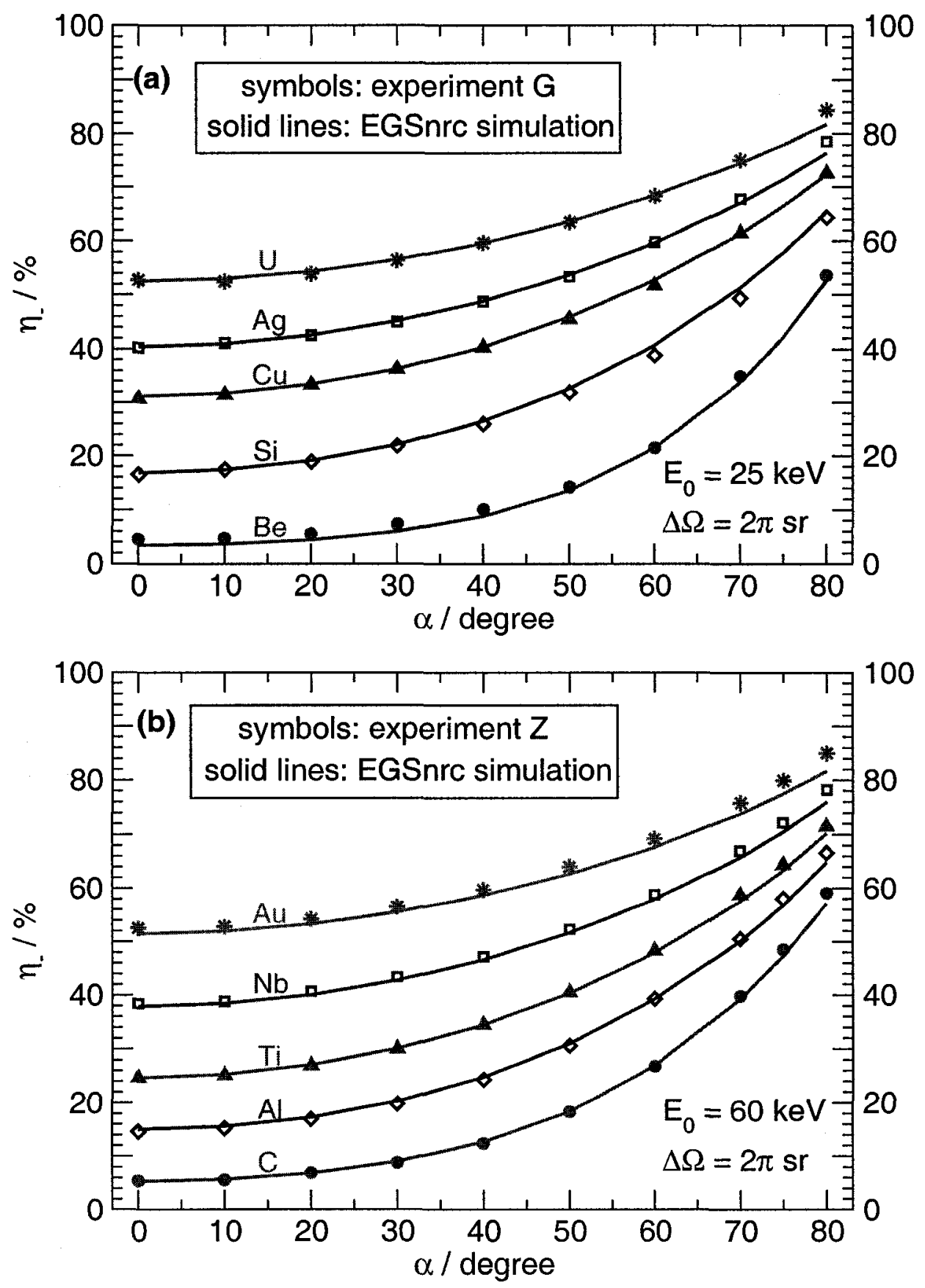

Figure 3.5: EGSnrc results versus experimental data for the variation of the total electron backscatter coefficient, $\eta_{-}$, with the angle of incidence of the electron beam, $\alpha$. EGSnrc results are connected by solid line segments without symbols for graph clarity. Experimental data are from experiments with indices $G$ and $Z$. 
be attributed to the issues associated with near-grazing incidence which were discussed in section 3.3 (i.e., incident beam masking, detector masking, surface contamination, ... etc).

\subsubsection{Energy spectra}

Figure 3.6 compares EGSnrc results versus experimental data for the overall energy spectra of electrons backscattered from semi-infinite targets of $\mathrm{Al}, \mathrm{Cu}, \mathrm{Ag}$ and Au. Given the variation between experiments in the lower portion of the spectra, it can be said that the EGSnrc prediction of the spectra of backscattered charged particles is in agreement with experimental data for all Z. As Z increases, the spectra peak closer to the original incident energy, and consequently their mean energy increases. The slight energy shift between different experiments could be due to energy calibration uncertainties. Some experiments ${ }^{53}$ report an overall uncertainty of $\sim 10 \%$, but the observed variations among experiments in the lower portion of the spectra are much larger. This could be due to the poorer detector resolution in that energy range (see section 3.3 ).

Panel a of figure 3.7 compares EGSnrc results versus experimental data for the variation of the overall energy spectra of backscattered electrons with the angle of incidence, $\alpha$. Given the poor resolution of the data digitized from the article by Kanter, ${ }^{61}$ it can be said that EGSnrc tracks the experimental data well. For normal incidence, the electrons penetrate the deepest and lose the most energy compared to larger incidence angles. This is demonstrated by the broad spectrum at $\alpha=0^{\circ}$, which gets narrower and peaks towards higher energies as $\alpha$ increases. The area under the curves also increases with $\alpha$ following the same argument presented in figure 3.5 . 

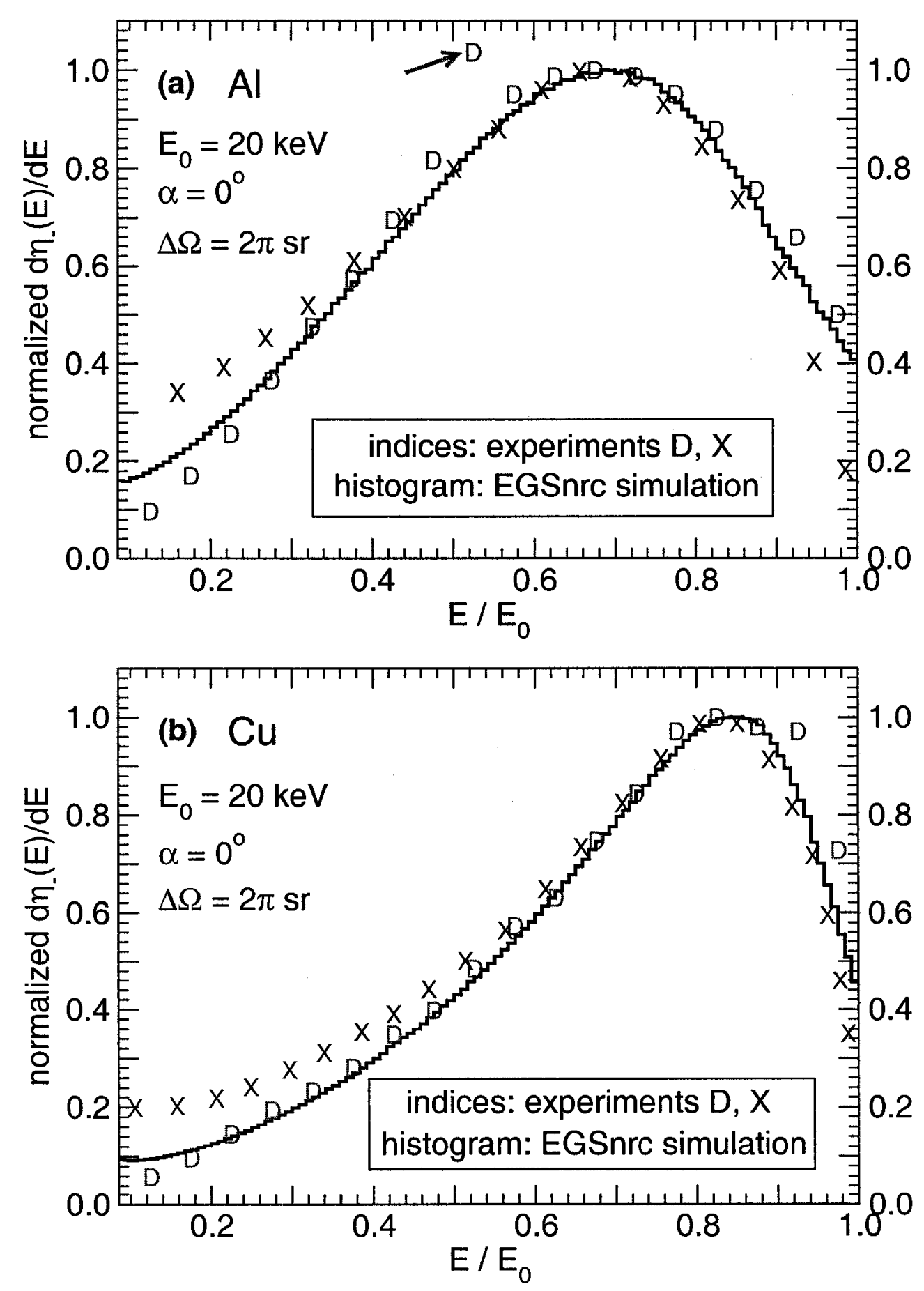

3.5. RESULTS 

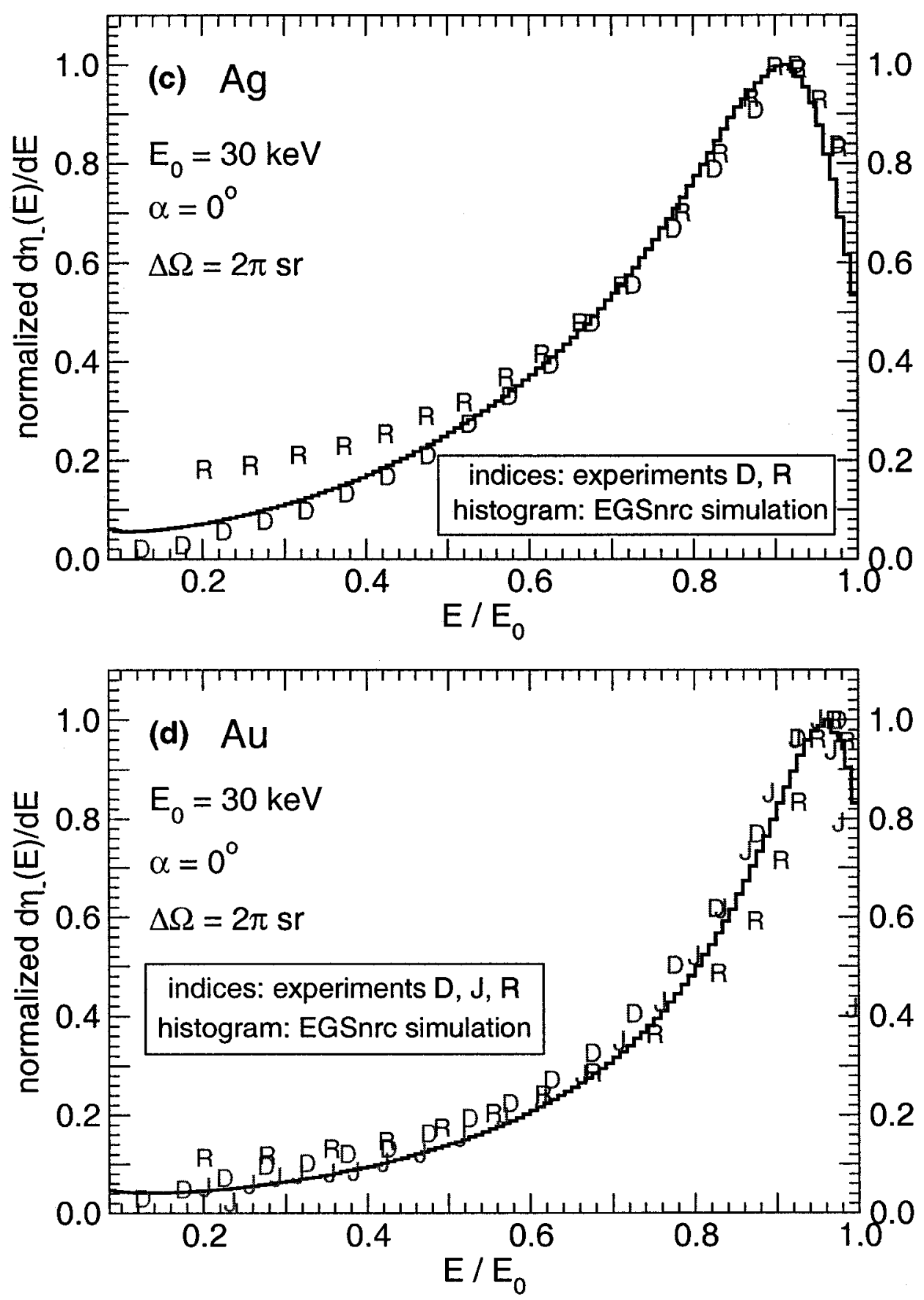

Figure 3.6: EGSnrc results versus experimental data for the overall energy spectra of electrons backscattered from semi-infinite targets of $\mathrm{Al}, \mathrm{Cu}, \mathrm{Ag}$ and $\mathrm{Au}$. Experimental data are taken from experiments with indices $D, J, R$ and $X$. See figure 3.1 and table 3.1 for definition of the axes labels and other symbols. In panel a, the anomalous point labeled with an arrow could be a typo in table 3 by Darlington, ${ }^{51}$ or it could be due to experimental instabilities of the energy recorder. Data below $E / E_{0} \sim 0.1$ are either unavailable or too noisy. 

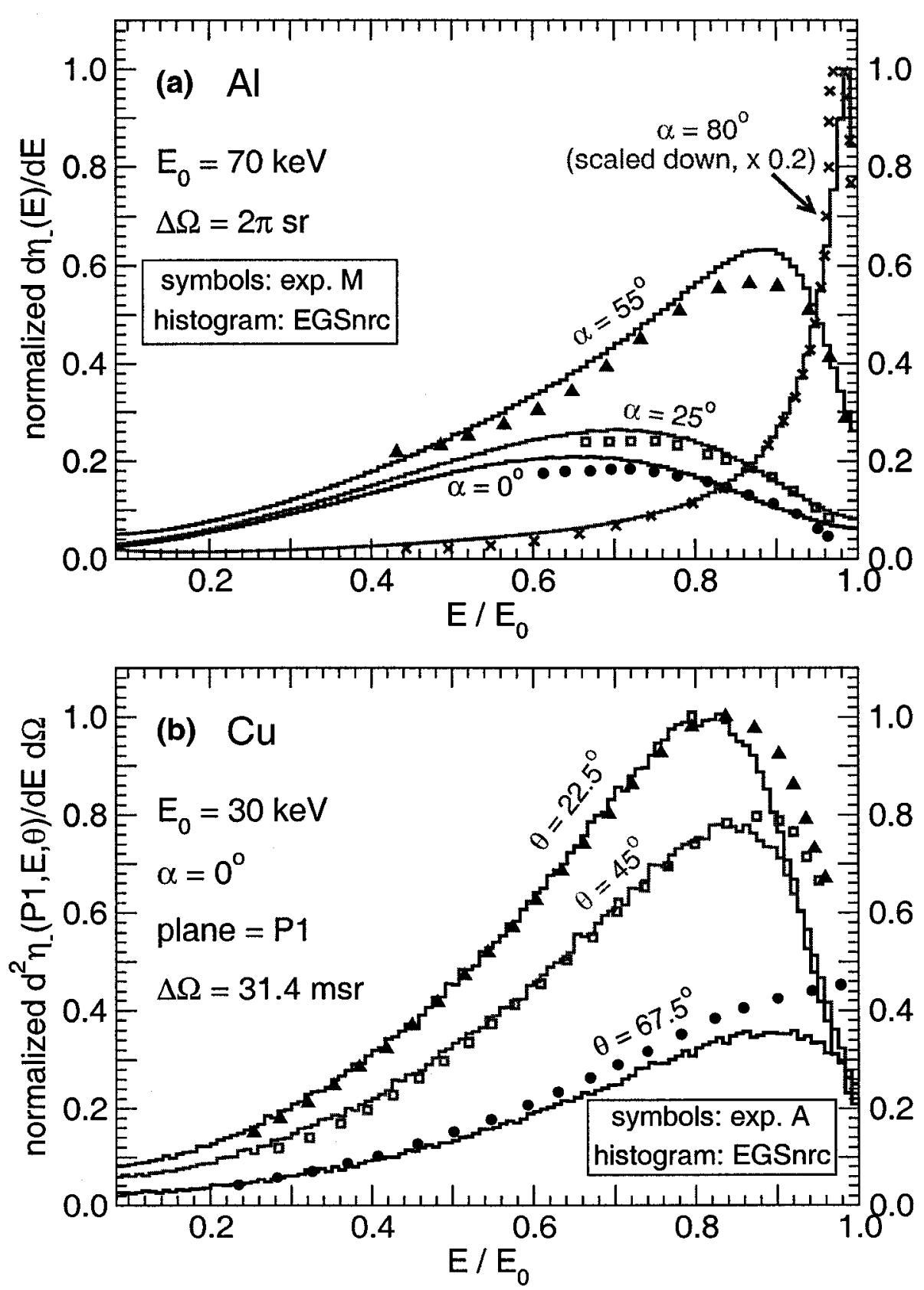

Figure 3.7: EGSnrc results versus experimental data for the variation of the overall energy spectra of backscattered electrons with the angle of incidence, $\alpha$, in panel $a$, and the variation of the localized energy spectra with the backscattering angle, $\theta$, in panel $b$. Experimental data are taken from experiments with indices $A$ and M. In panel a for $\alpha=80^{\circ}$, both experimental data and EGSnrc results are scaled down by a factor of 5 for clarity of other curves. For $\alpha=0^{\circ}$, the resolution of experimental data is poor, and it was hard to faithfully digitize the data. Note that the ordinate in panel $a$ is different from that in panel $b$. 
Panel b of figure 3.7 compares EGSnrc results versus experimental data for the variation of the spectra of backscattered electrons with the backscatter angle, $\theta$. EGSnrc reproduces the experimental measurements reasonably well. For normal incidence, the differential probability for backscattering at a larger $\theta$ before losing much energy is higher than that at a smaller $\theta$. This makes the energy spectrum at $\theta=67.5^{\circ}$ peak closest to the original incident energy $E_{0}$. However, the number of electrons backscattered at larger $\theta$ is smaller than that at smaller $\theta$, and so the relative area under the curve of $\theta=67.5^{\circ}$ is the smallest. The energy shift between the experimental data and EGSnrc simulations could be due to energy calibration issues. At the near-grazing backscatter angle of $\theta=67.5^{\circ}$, the uncertainties are larger than those for smaller $\theta$. Also, geometrical and alignment issues can play a role in explaining the differences.

Figure 3.8 gives the difference, in keV, between EGSnrc results and experimental data for the most probable energy (panel a), and for the mean energy (panel b) of the energy spectra of backscattered electrons. For fair comparison, the integration to calculate the mean energy starts at $\mathrm{E} / \mathrm{E}_{0}=0.2$ for the experimental and simulation data. The mean energy for comparison purposes is given by:

$$
E_{\text {mean }}=\frac{\sum_{i=25}^{120} E_{i}\left(\frac{d \eta_{-}}{d E_{i}}\right)_{E_{i}}}{\sum_{i=25}^{120}\left(\frac{d \eta_{-}}{d E_{i}}\right)_{E_{i}}}
$$

Figure 3.8 indicates that EGSnrc can reproduces the experimental values of the most probable and mean energies to within $\pm 1.5 \mathrm{keV}$ at $\mathrm{E}_{0}=30 \mathrm{keV}$ (i.e. within $\pm 5 \%$ ).

3.5. RESULTS 

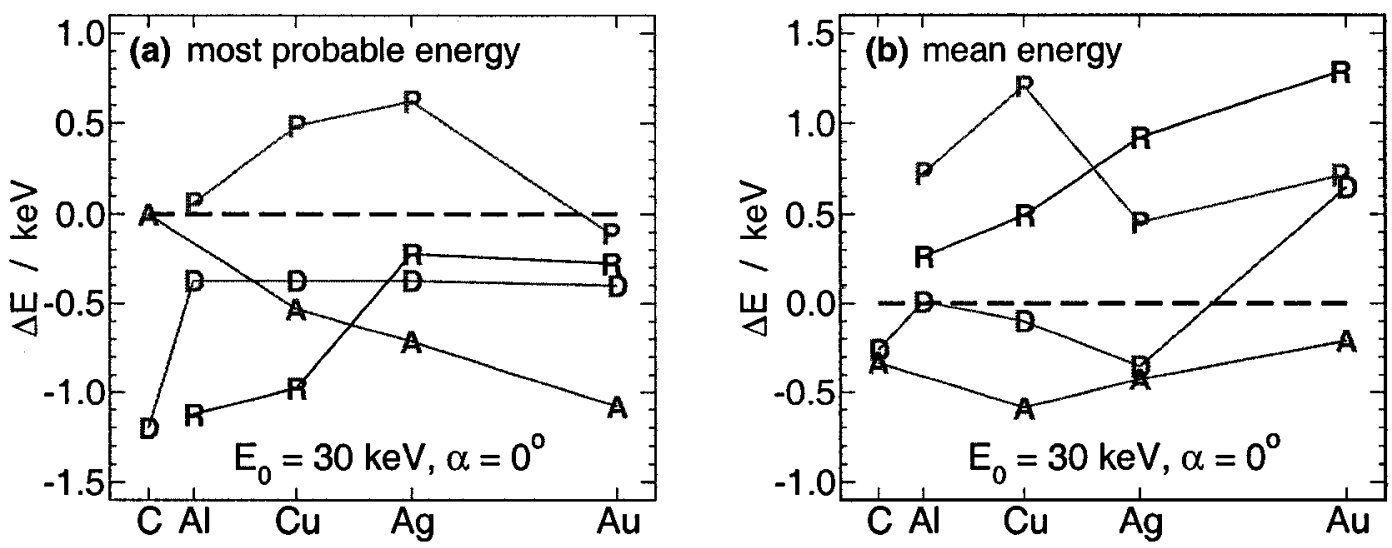

Figure 3.8: The difference (in keV) between EGSnrc results and experimental data for the most probable energy (panel a), and for the mean energy (panel b) of the energy spectra of backscattered electrons. Experimental data are taken from experiments with indices $A, D, P$ and $R$. The line segments in the figure are merely to aid in tracking a particular experiment. Experiments $D$ and $R$ measure the overall energy spectrum (i.e. $\Delta \Omega=2 \pi \mathrm{sr}$ ), while experiments $\mathrm{A}$ and $\mathrm{P}$ measure a localized energy spectrum in plane $P 1$ at $\theta=45^{\circ}$ and $43^{\circ}$, with $\Delta \Omega=31.4$ and $4.81 \mathrm{msr}$, respectively. Uncertainty in determining the most probable energy is higher for lower- $Z$ targets because the peaks are much broader.

\subsubsection{Angular distribution}

Figure 3.9 compares EGSnrc results versus experimental data for the angular distribution in plane P1 for normally-incident electrons backscattering from semiinfinite targets of $\mathrm{Cu}$ and $\mathrm{Au}$. For both low- and high-Z targets, the agreement between EGSnrc results and experimental data is within the experimental uncertainties. The angular distribution at normal incidence exhibits a cosine-like distribution, i.e. $d \eta_{-}(P x, \theta) / d \Omega$ varies almost linearly with $\cos \theta$, which shows as a circle in the polar plots. At large $\theta$ (close to $90^{\circ}$ and $-90^{\circ}$ ), the angular distribution deviates slightly from a perfect cosine. For normal incidence, the angular distribution does not vary much with $\mathrm{Z}$ (the angular distributions for $\mathrm{Cu}$ and $\mathrm{Au}$ are almost the same). 

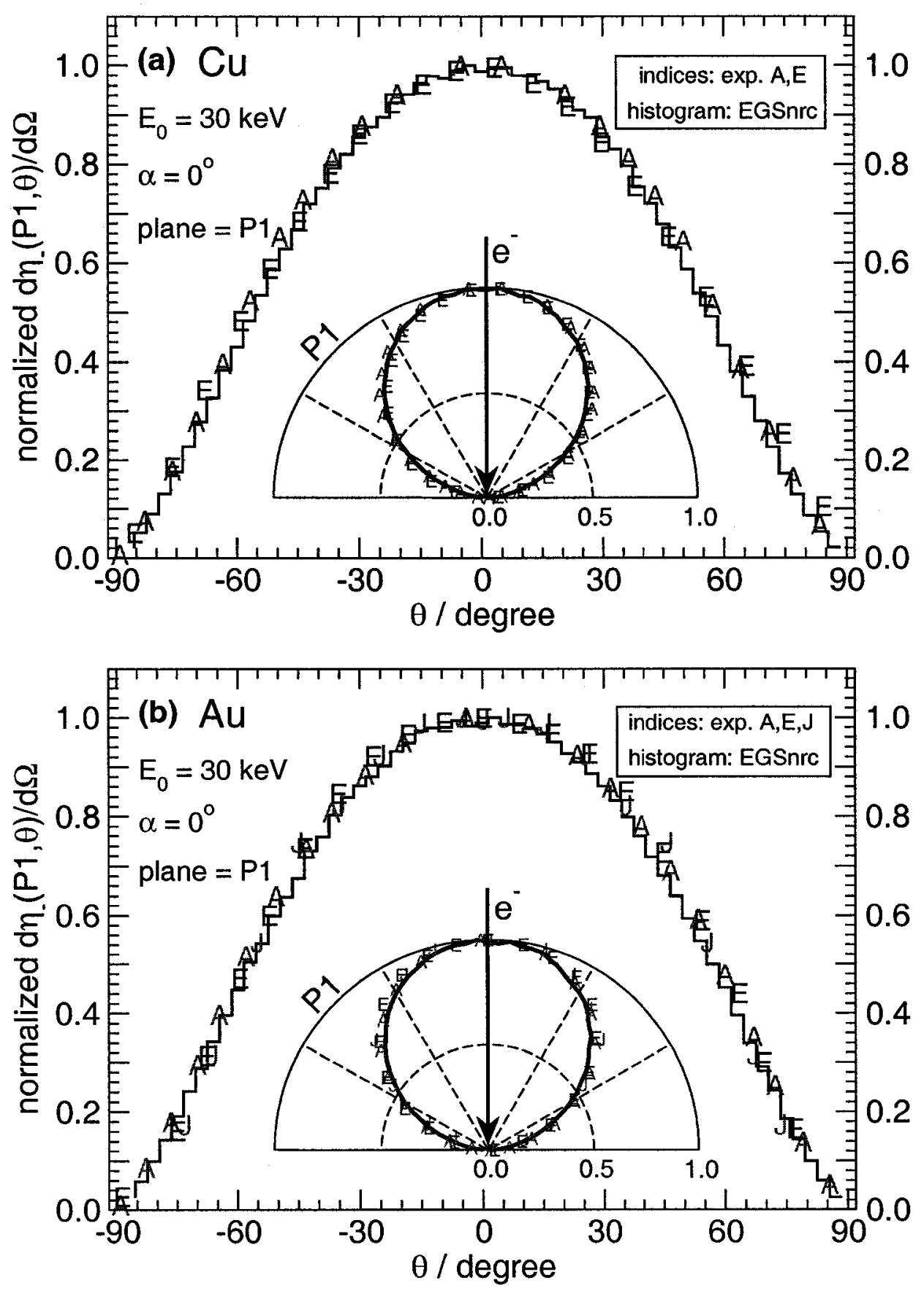

Figure 3.9: EGSnrc results versus experimental data for the angular distribution of electrons backscattered from semi-infinite targets of $\mathrm{Cu}$ and $\mathrm{Au}$. Experimental data are taken from experiments with indices $A, E$ and $J$. Results are presented in Cartesian and polar form for both elements. $\Delta \Omega=31.4,1.9$ and $0.9 \mathrm{msr}$ for experiments $A, E, J$, respectively. 
Figure 3.10 compares EGSnrc results versus experimental data for the variation of the angular distribution with $\mathrm{Z}$ for oblique incidence. EGSnrc reproduces the experiment well except for a normalization and a slight peak shift issue with carbon, which could be due to alignment uncertainties or other systematic changes in the experiment when target materials are changed. Unlike normal incidence, the angular distribution at oblique incidence varies dramatically with $\mathrm{Z}$.

Figure 3.11 compares EGSnrc results versus experimental data for the variation of the angular distribution with $\alpha$. EGSnrc reproduces the experiment very well, except for $\mathrm{Ag}$ at $\alpha=30^{\circ}$. The number of backscattered electrons per unit solid angle peaks near the "reflection" or "mirror" angle of $\alpha$. This trend becomes more obvious as $\alpha$ increases. The small discrepancy at grazing incidence $\left(\alpha=80^{\circ}\right)$ can be attributed to the uncertainties associated with grazing incidence, which were discussed in section 3.3 .

Most angular distribution measurements are done with the plane of backscatter being the same as the plane of incidence (both are plane P1). Very few experimental data are available for angular distributions in backscatter planes other than P1. Figure 3.12 compares EGSnrc results versus experimental data for the variation of the angular distribution with the plane of backscatter, $\mathrm{Px}$, for $\mathrm{Al}$ and Au at oblique incidence. EGSnrc reproduces the experiment reasonably well, with the agreement being better for $\mathrm{Al}$ than it is for $\mathrm{Au}$. The feature that the distribution peaks at the "mirror" angle of $\alpha$ is evident in P1 for Al. As P1 rotates $45^{\circ}$ to become $\mathrm{P} 2$, the angular distribution gets broader. As P2 rotates another $45^{\circ}$ to become P3 and perpendicular to the plane of incidence, P1, the angular distribution becomes symmetric around $\theta=0^{\circ}$. In the polar plots, as the plane of backscatter rotates from P1 to P3, the cigar-like angular distribution gradually changes to a pancake-like one.

3.5. RESULTS 

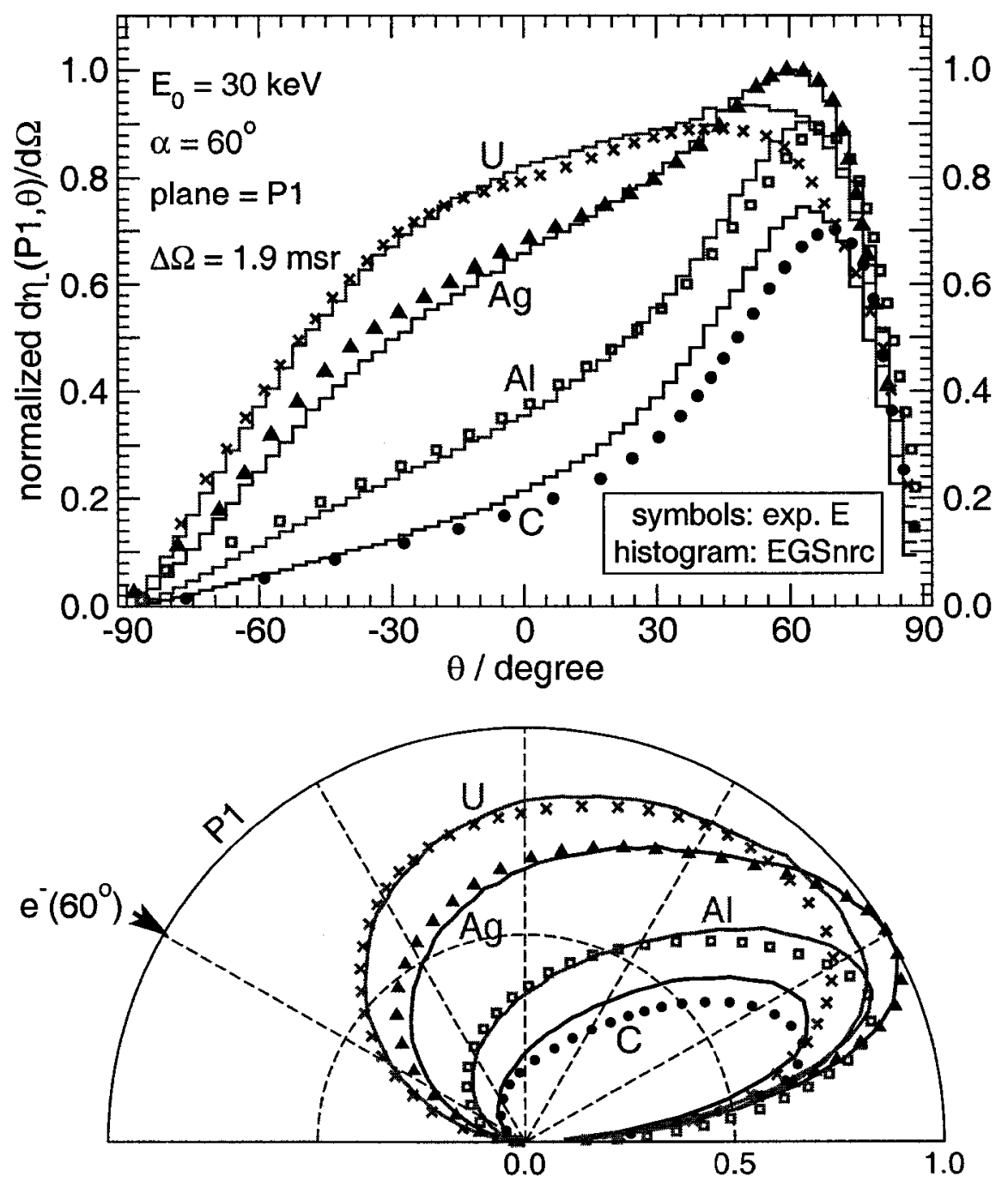

Figure 3.10: EGSnrc results versus experimental data from experiment $E$ for the variation of the angular distribution of backscattered electrons with the atomic number of the target material, $Z$, at oblique incidence. 

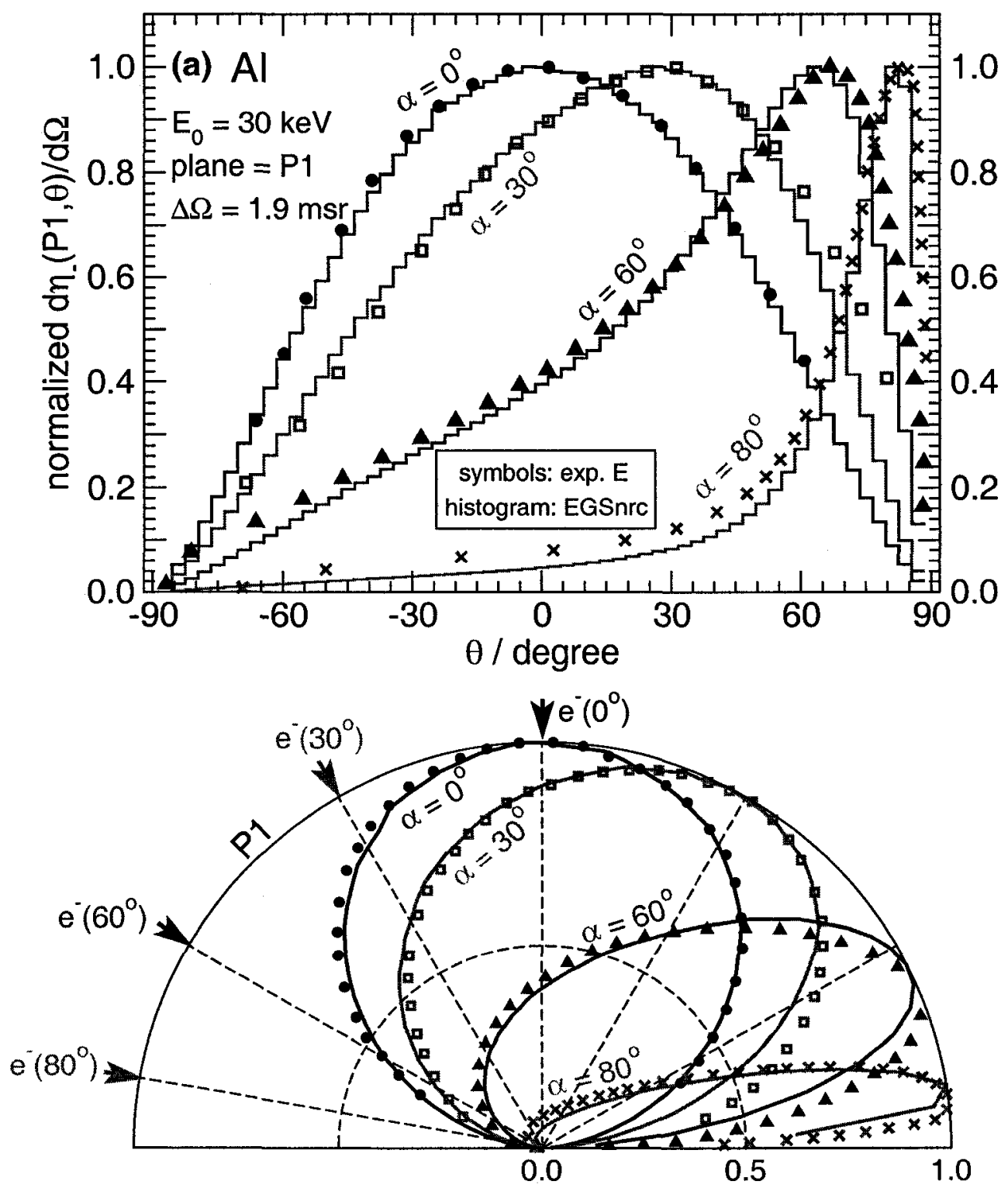

3.5. RESULTS 

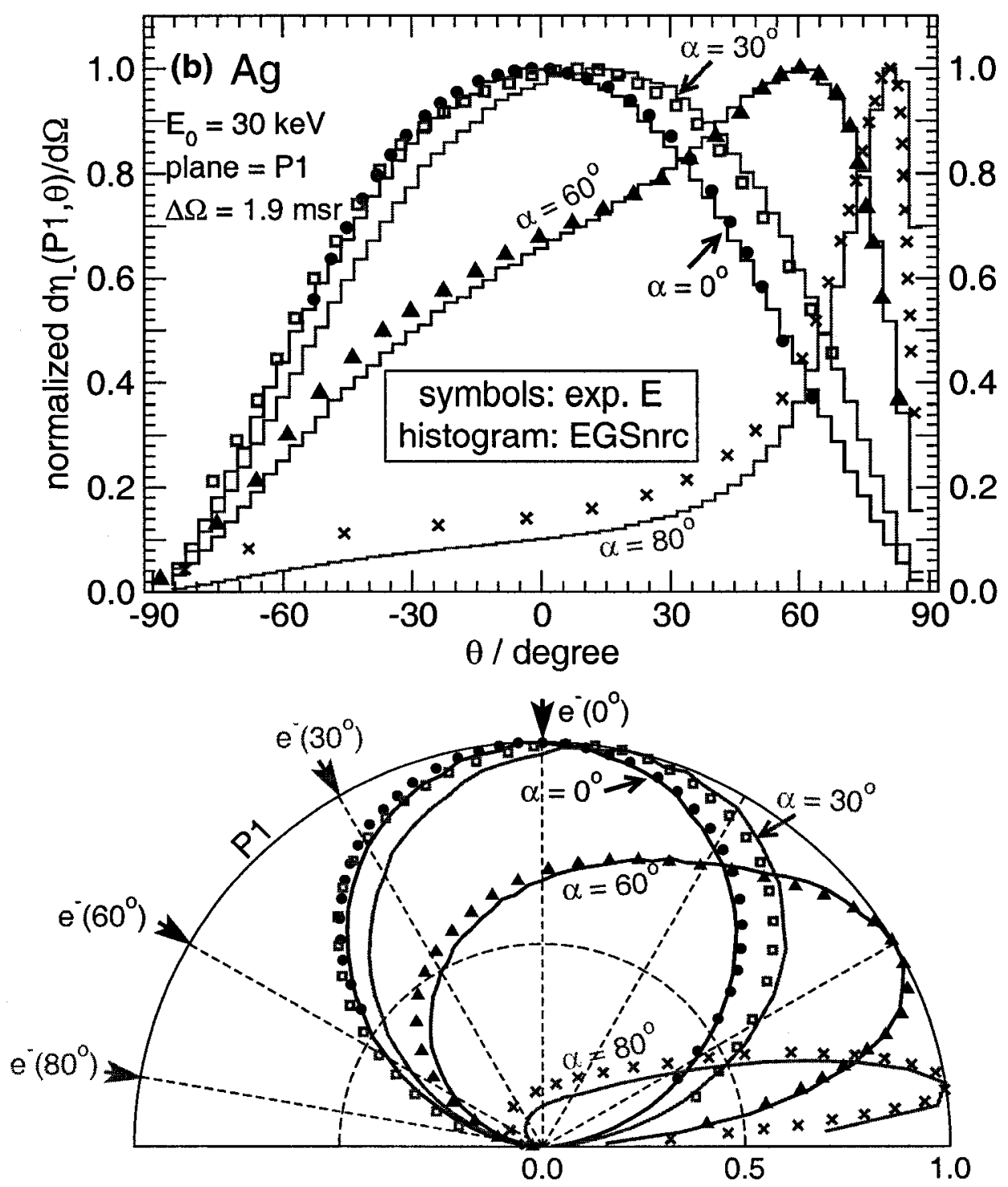

Figure 3.11: EGSnrc results versus experimental data for the variation of the angular distribution of backscattered electrons with the angle of incidence, $\alpha$, for $\mathrm{Al}$ and $\mathrm{Ag}$. Experimental data are taken from multiple graphs in experiment $\mathrm{E}$, each for a particular $\alpha$. Experiment $\mathrm{E}$ does not provide a common normalization between different $\alpha$, consequently all curves in this figure had to be normalized to unity at their peak. Unlike other graphs in this study, the areas under the curves in this figure do not represent a measure of the relative total backscatter. 

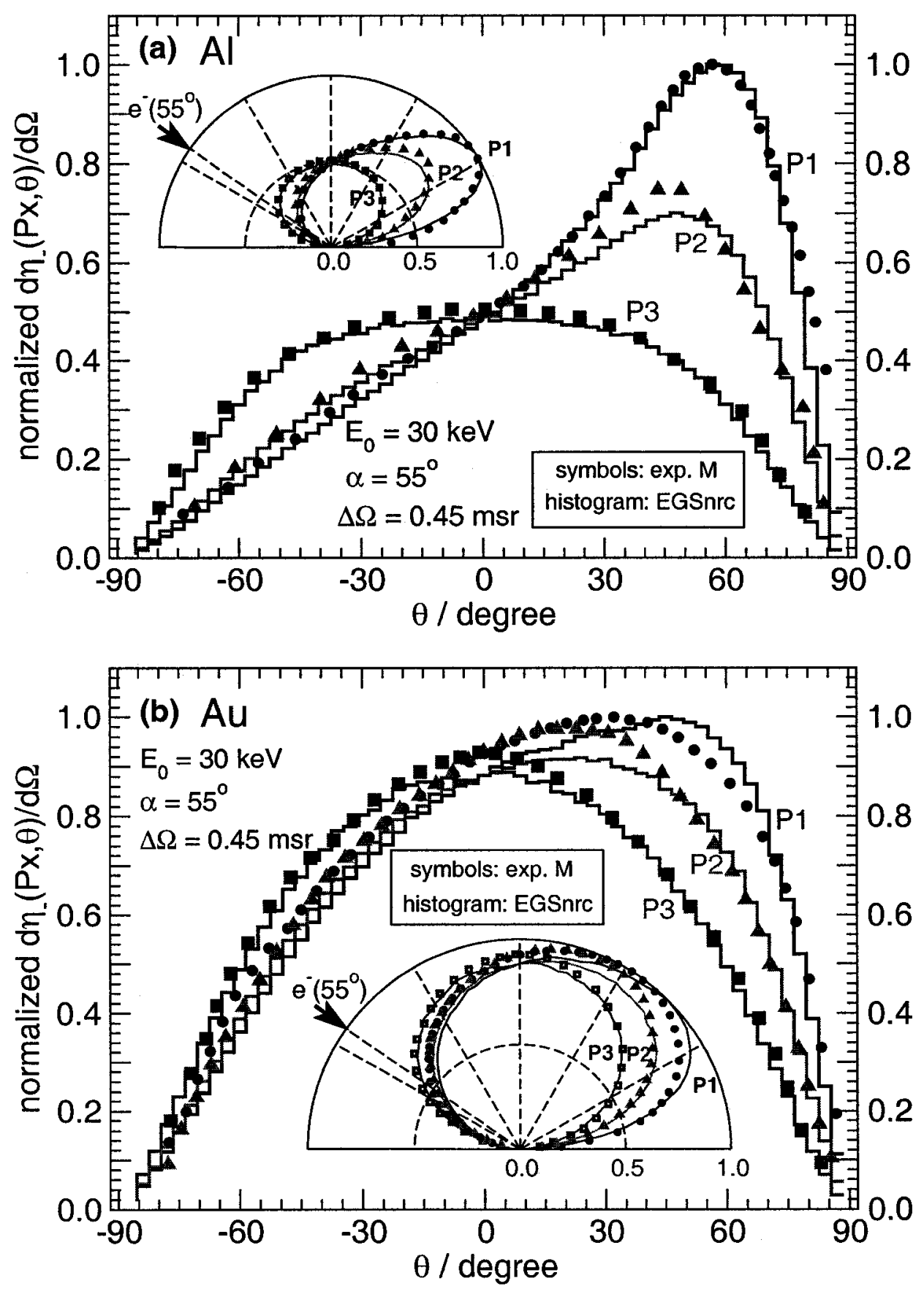

Figure 3.12: EGSnrc results versus experimental data for the variation of the angular distribution of backscattered electrons with the plane of backscatter, $\mathrm{Px}$, for oblique incidence on semi-infinite targets of $\mathrm{Al}$ and $\mathrm{Au}$. Experimental data are taken from experiment $M$. Unlike all other polar plots in this study, the plane of the page in this figure does not represent a particular backscatter plane. 


\subsubsection{Backscatter from thin films}

As discussed in section 3.1, accurate prediction of dose perturbation due to thin layers of high- $\mathrm{Z}$ inhomogeneities in tissue is strongly affected by the accuracy of simulating the backscatter of charged particles from these thin layers. ${ }^{46,49}$ For this reason, the capabilities of EGSnrc to simulate backscatter from thin films is investigated in this section.

Panels $\mathrm{a}$ and $\mathrm{b}$ in figure 3.13 compare EGSnrc results versus experimental data for the variation of $\eta_{-}$with the film thickness for various $E_{0}$ for $\mathrm{Cu}$ and $\mathrm{Au}$. The experiments are designed such that the thin films are self-supporting. ${ }^{55}$ The EGSnrc data shows an almost-linear increase of $\eta_{-}$with film thickness at different energies for both $\mathrm{Cu}$ and $\mathrm{Au}$; the increase is steeper for lower $E_{0}$. As the film thickness increases, $\eta_{-}$approaches the value for bulk targets and then saturates. The saturation occurs at smaller film thicknesses for lower $E_{0}$ because the ratio of the film thickness to the range of the incident electrons in the target material increases as $E_{0}$ decreases. At $20 \mathrm{keV}$, the EGSnrc value of the film thickness at which $\eta_{-}$reaches half its saturation value, divided by the CSDA range of electrons in the target material ${ }^{76}$ is $\sim 0.1$ and $\sim 0.07$ for $\mathrm{Cu}$ and $\mathrm{Au}$, respectively. This indicates that about half of the backscattered electrons are backscattered from within the first $10 \%$ of their full penetration depth.

The variation among different experimental data in panels $a$ and $b$ of figure 3.13 is more dramatic than it is for bulk materials because measuring backscatter from thin films is fraught with more uncertainties than backscatter from bulk materials is (e.g. exact film thickness, effect of substrate material, more sensitivity to surface contamination). Given these facts, it can be said that EGSnrc reproduces the experimental "trends" well. The large discrepancy at $E_{0}=20$ and

3.5. RESULTS 

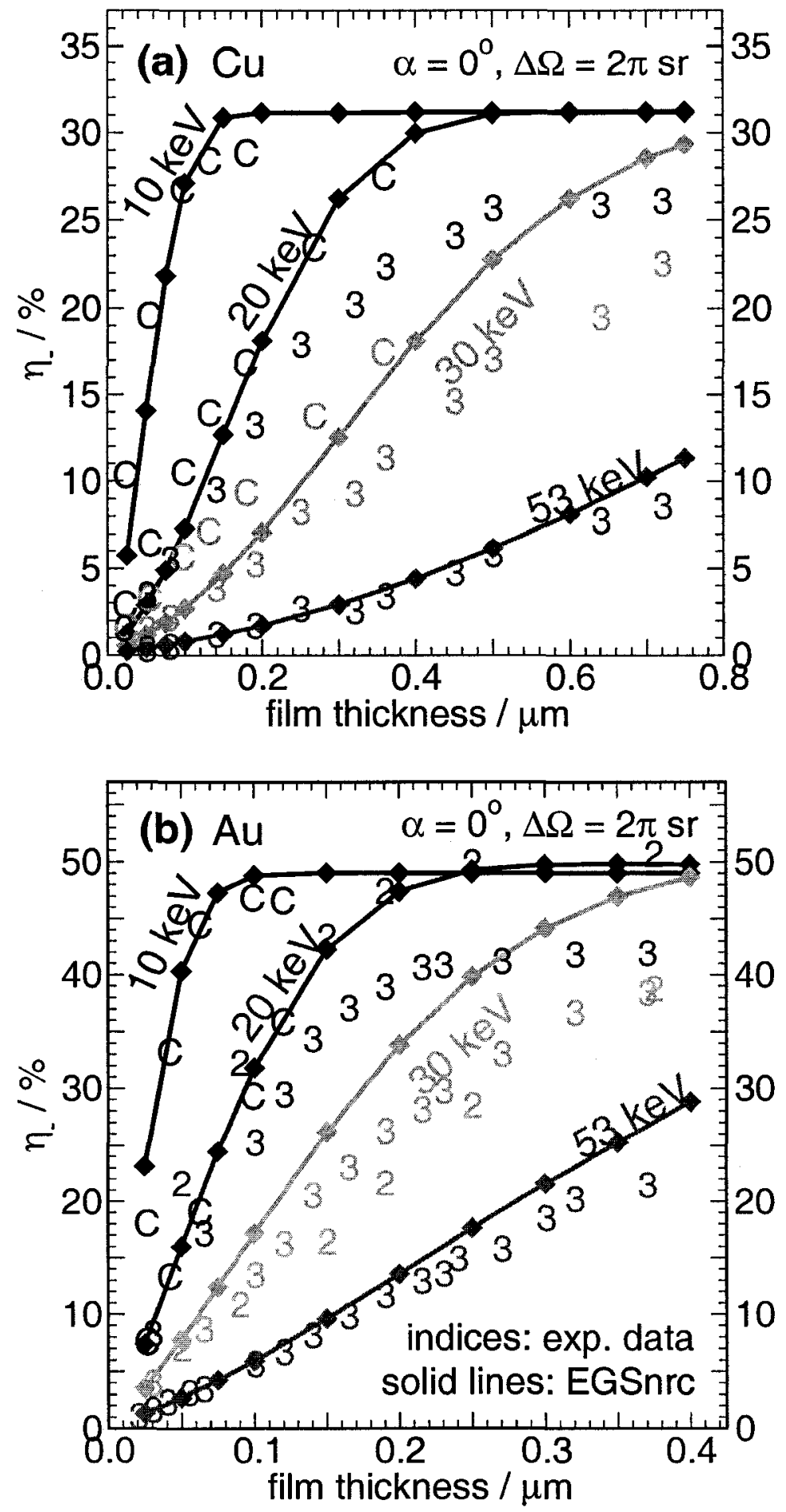

Figure 3.13: Backscatter from thin films: EGSnrc results versus experimental data for the variation of electron backscatter coefficient with the incident electron energy. Experimental data are taken from experiments with indices C, 2 and 3. EGSnrc results (filled diamonds) are connected by solid line segments. 
$30 \mathrm{keV}$ is most likely due to systematic uncertainties in the experiments. This conclusion is drawn because at large film thicknesses, a few of the experimental datasets saturate at much lower values than those reported for bulk targets by many other experiments at the same energies. For example, from figure 3.3 page 54, for backscatter from bulk targets at $30 \mathrm{keV}$, both EGSnrc and experimental data predict $\eta_{-}$values of $\sim 31 \%$ and $\sim 50 \%$ for $\mathrm{Cu}$ and $\mathrm{Au}$, respectively. However, the experimental data in panels $a$ and $b$ of figure 3.13 saturate at $\sim 26 \%$ and $\sim 42 \%$, respectively.

Panel a in figure 3.14 compares EGSnrc results versus experimental data for the localized energy spectrum of electrons backscattered at $\theta=25^{\circ}$ in plane $\mathrm{P} 1$ from a $0.1 \mu \mathrm{m} \mathrm{Cu}$ film deposited on an $\mathrm{Al}$ substrate. EGSnrc reproduces the features of the energy spectrum very well, except for the magnitude of the high energy peak. Panel b in figure 3.14 compares EGSnrc results versus experimental data for the angular distribution of electrons backscattered in plane P1 from a composite film of $20 \mathrm{~nm}$ of $\mathrm{Au}$ followed by $20 \mathrm{~nm}$ of $\mathrm{Al}_{2} \mathrm{O}_{3}$ at different $E_{0}$. Because only one experiment is available, and with a very limited number of data points, only a qualitative statement can be made that EGSnrc is capable of predicting the angular distribution of backscattered electrons from composite thin films. The relative amplitude of the backscatter between $E_{0}=10$ and $15 \mathrm{keV}$ is very well predicted by EGSnrc.

3.5. RESULTS 

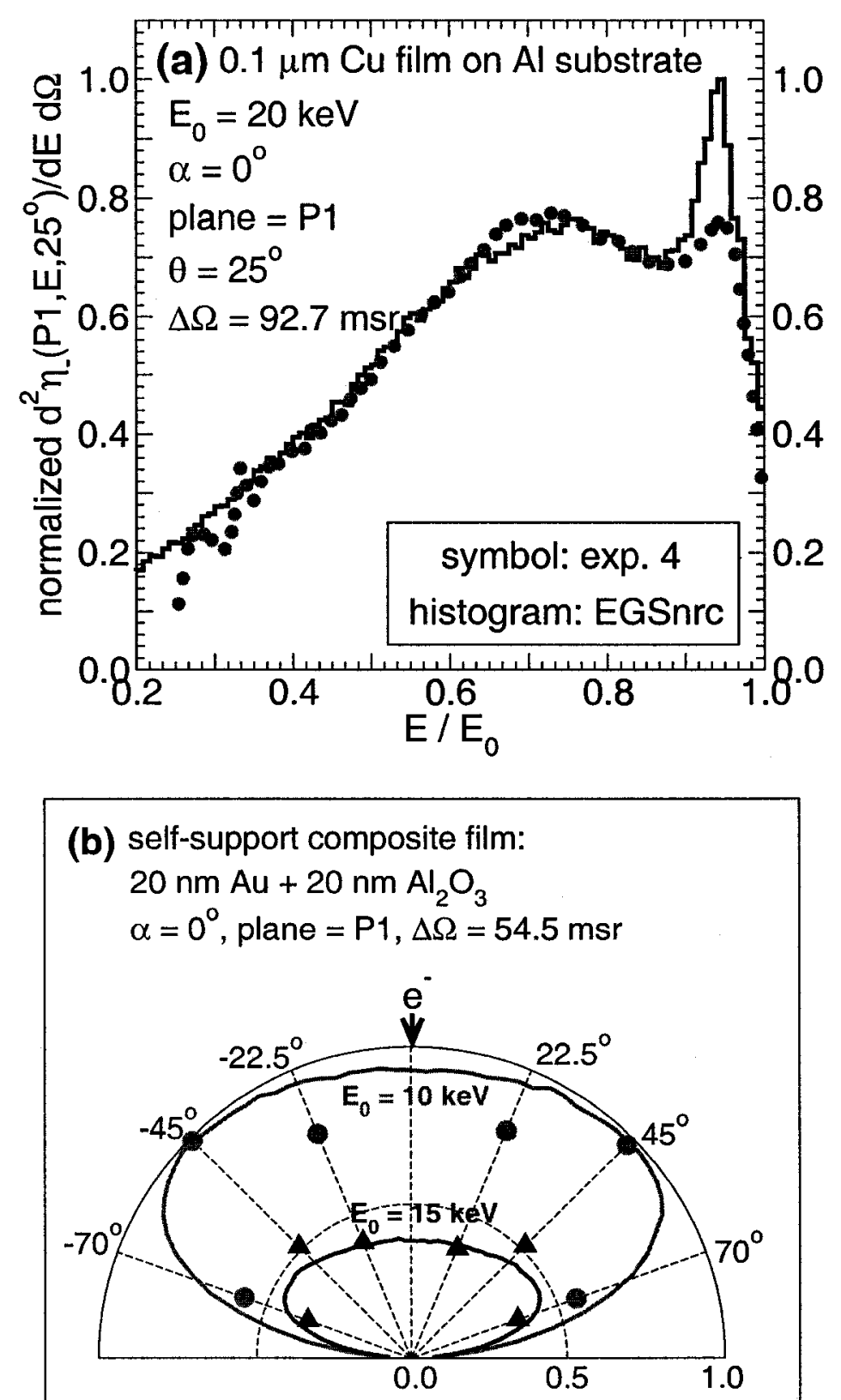

symbols: exp. N, solid lines: EGSnrc

Figure 3.14: Backscatter from thin films: EGSnrc results versus experimental data for the energy spectra and angular distribution of backscattered electrons. Experimental data are taken from experiments with indices $\mathrm{N}$ and 4 . In panel a, experimental data starts at $E / E_{0}=0.25$, and the data are noisy up to $E / E_{0} \sim 0.375$. Normalization in panel a is arbitrary (i.e. not largest-peak to largest-peak). In panel $b$, only 6 experimental data points are available at each energy. Also in panel b, the hole openings for collecting backscattered electrons vary slightly between data points, and the average is used to determine $\Delta \Omega$ for the EGSnrc simulation.

\subsection{RESULTS}




\subsubsection{Positron backscatter}

Unlike some of the other major Monte Carlo codes, EGSnrc always explicitly takes into account the difference in the stopping power and the inelastic scattering cross section between positrons and electrons. ${ }^{5,77}$ Positron transport can be important in certain Positron Emission Tomography (PET) simulations. This section examines the capabilities of EGSnrc to reproduce experimental measurements of backscattering of kilovoltage positrons from solid targets. Only monoenergetic positron beams are considered. Benchmarking point sources with an energy spectrum (e.g. $\beta^{+}$radioactive sources) is expected to yield similar agreement since it is merely a superposition of multiple monoenergetic sources. In most positron experiments, backscatter is indirectly estimated from measurements of the annihilation count rate of positrons decaying in the target (i.e., those that did not backscatter). The uncertainty associated with this technique is that energetic positrons backscattered at large angles may annihilate in front of the detector and get recorded as if they occurred in the target. This leads to under-estimation of $\eta_{+}$, which gets worse as $E_{0}$ increases. ${ }^{54}$

Similar to figures 3.3 through 3.5 for electrons, figure 3.15 compares EGSnrc results versus experimental data for the variation of $\eta_{+}$with $E_{0}, \mathrm{Z}$ and $\alpha$. The amount of experimental data available for positron backscatter is much less than for electron backscatter, and it covers a narrower energy range. In panel a, EGSnrc simulations can predict the energy dependence of $\eta_{+}$well. The energy dependence is more pronounced than it is for electrons. In panels b and c, EGSnrc simulations can replicate the monotonic increase of $\eta_{+}$with $\mathrm{Z}$ and $\alpha$. Other arguments presented for electrons are also applicable to positrons.

3.5. RESULTS 

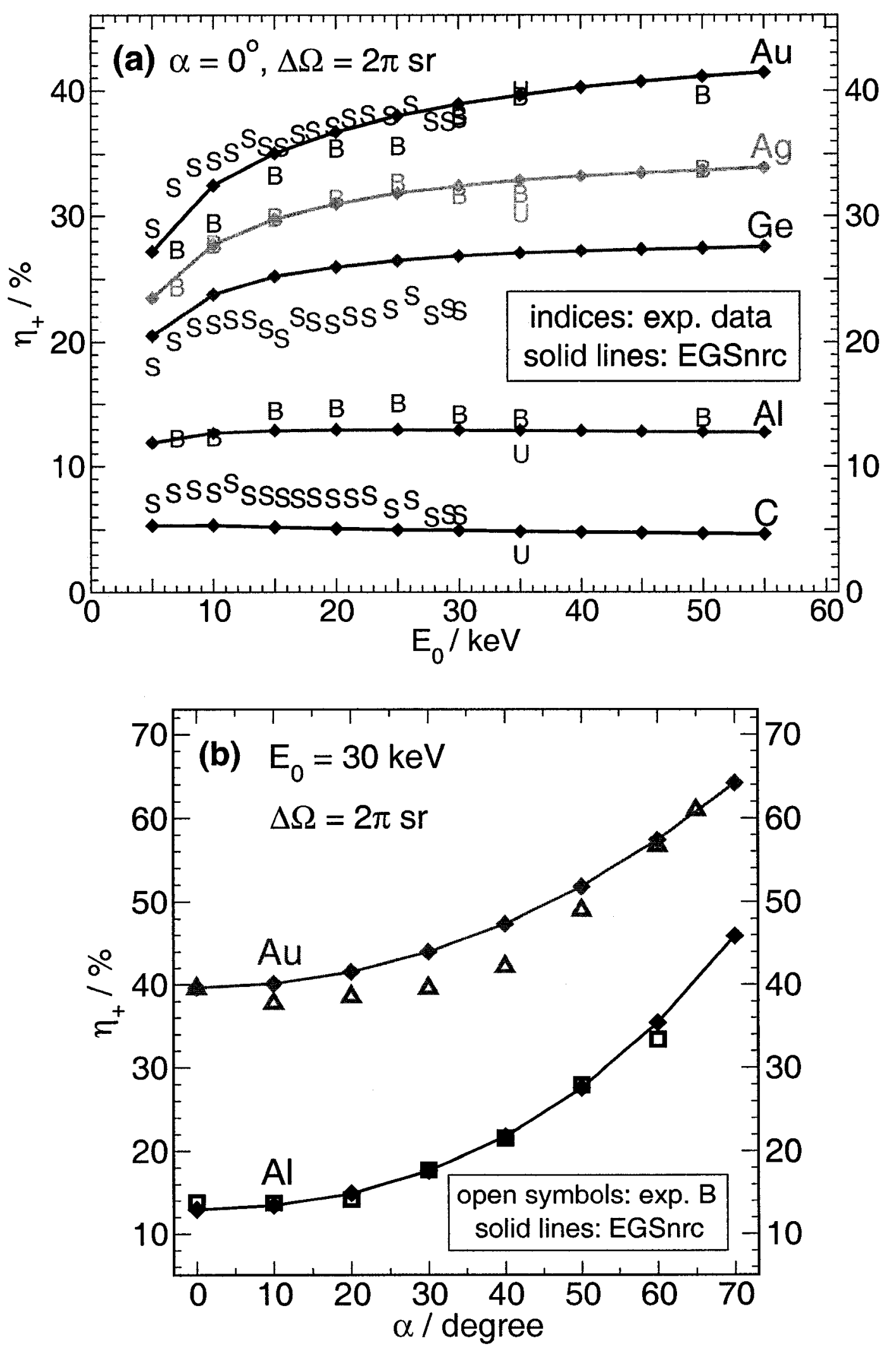

3.5. RESULTS 


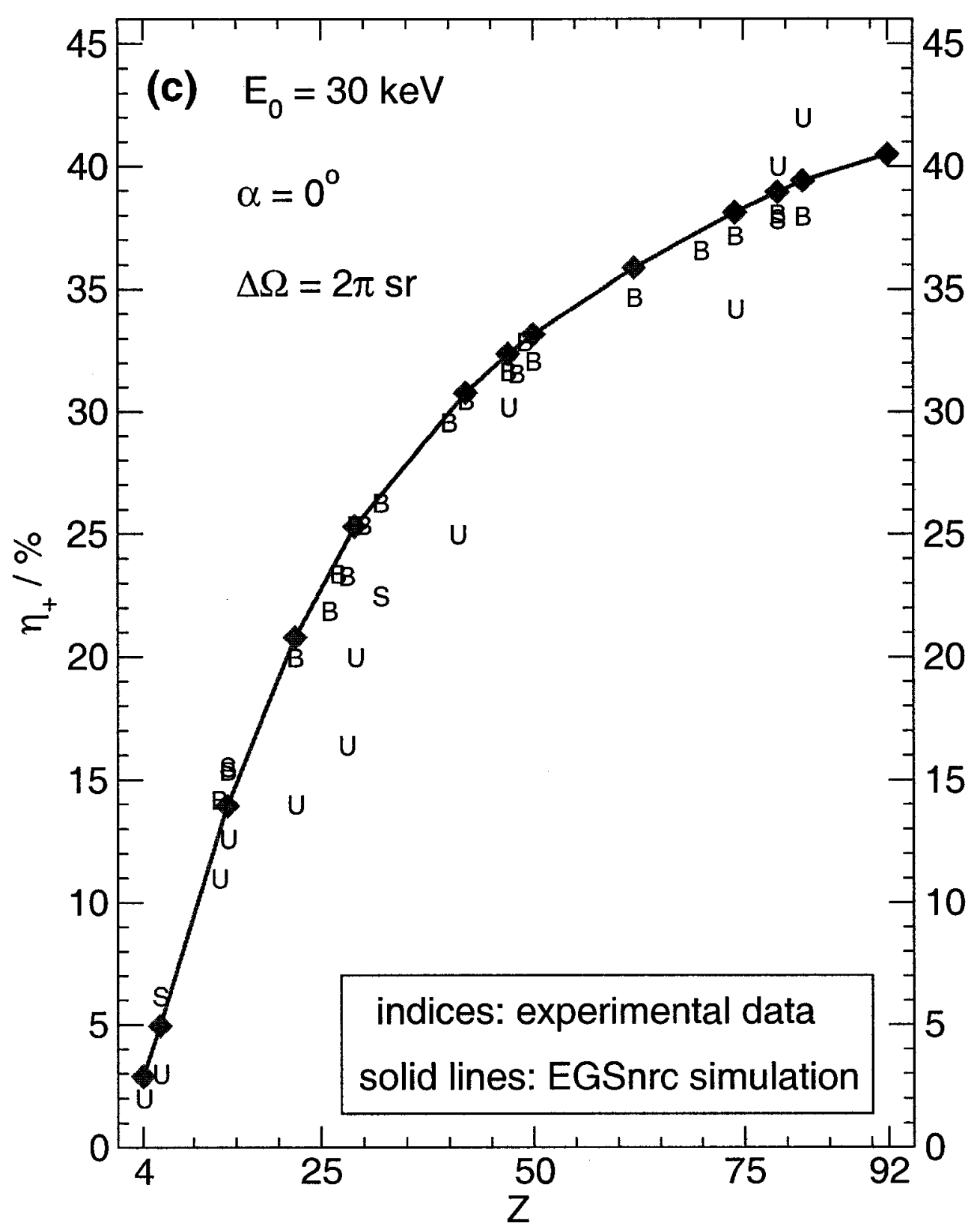

Figure 3.15: EGSnrc results versus experimental data for the variation of the total positron backscatter coefficient, $\eta_{+}$, with the incident energy of the positron beam, $E_{0}$ - panel a, with the angle of incidence of the positron beam, $\alpha$ - panel b, and with the atomic number of the target material, Z - panel c. EGSnrc results (filled diamonds) are connected by solid line segments. Experimental data are taken from experiments with indices $B, S$ and $U$. In panel a, experiment $U$ has only one data point at $35 \mathrm{keV}$ for $\mathrm{C}, \mathrm{Al}, \mathrm{Ag}$ and $\mathrm{Au}$. In panel b, experimental data at $\alpha=65^{\circ}$ are available for Au only. In panel $c, E_{0}$ in experiment $U$ is $35 \mathrm{keV}$, not $30 \mathrm{keV}$.

3.5. RESULTS 
Figure 3.16 compares EGSnrc results versus experimental data for the variation of the ratio $\eta_{-} / \eta_{+}$with $E_{0}$ and Z. The experimental ratios are obtained by dividing the results from different electron and positron experiments. This is because, except for the experiment with index $U$, no other experiment has performed both electron and positron backscatter measurements. The experiments chosen to calculate the ratio are the ones that have the same parameters for electrons and positrons (Z, $E_{0}$ and $\alpha$ ), with preference given to more complete electron datasets. Given the random nature of choosing pairs of experiments to obtain the ratio, it can be seen that EGSnrc results can predict the variation of $\eta_{-} / \eta_{+}$ with $E_{0}$ and $\mathrm{Z}$ very well. For the energy range shown, because elastic scattering is larger for electrons, and inelastic scattering is larger for positrons, the ratio $\eta_{-} / \eta_{+}$ is always $>1$ and increases slightly with $\mathrm{Z}$. The ratio is much higher at low $E_{0}$, and it saturates at about 1.3 as $E_{0}$ increases. This saturation value of 1.3 has often been reported in the literature from different attempts to estimate the ratio $\eta_{-} / \eta_{+}$using various experiment pairs. ${ }^{54,68}$ The ability of EGSnrc to predict these trends is a direct result of its explicit account of the differences in electron and positron transport.

Unlike electrons, there is a dearth of experimental data for the energy spectra and angular distribution of backscattered positrons. The only spectra that could be located are those by Massoumi et al, ${ }^{67}$ but they are convolved with the response function of the detector used. For the angular distribution, figure 3.17 compares EGSnrc results versus experimental data for the variation of the angular distribution of backscattered positrons with $\alpha$. As with electrons, EGSnrc reproduces the experimental data very well. The angular distributions of backscattered positrons qualitatively resemble those of backscattered electrons, and arguments similar to those made for electrons can be made for positrons.

3.5. RESULTS 

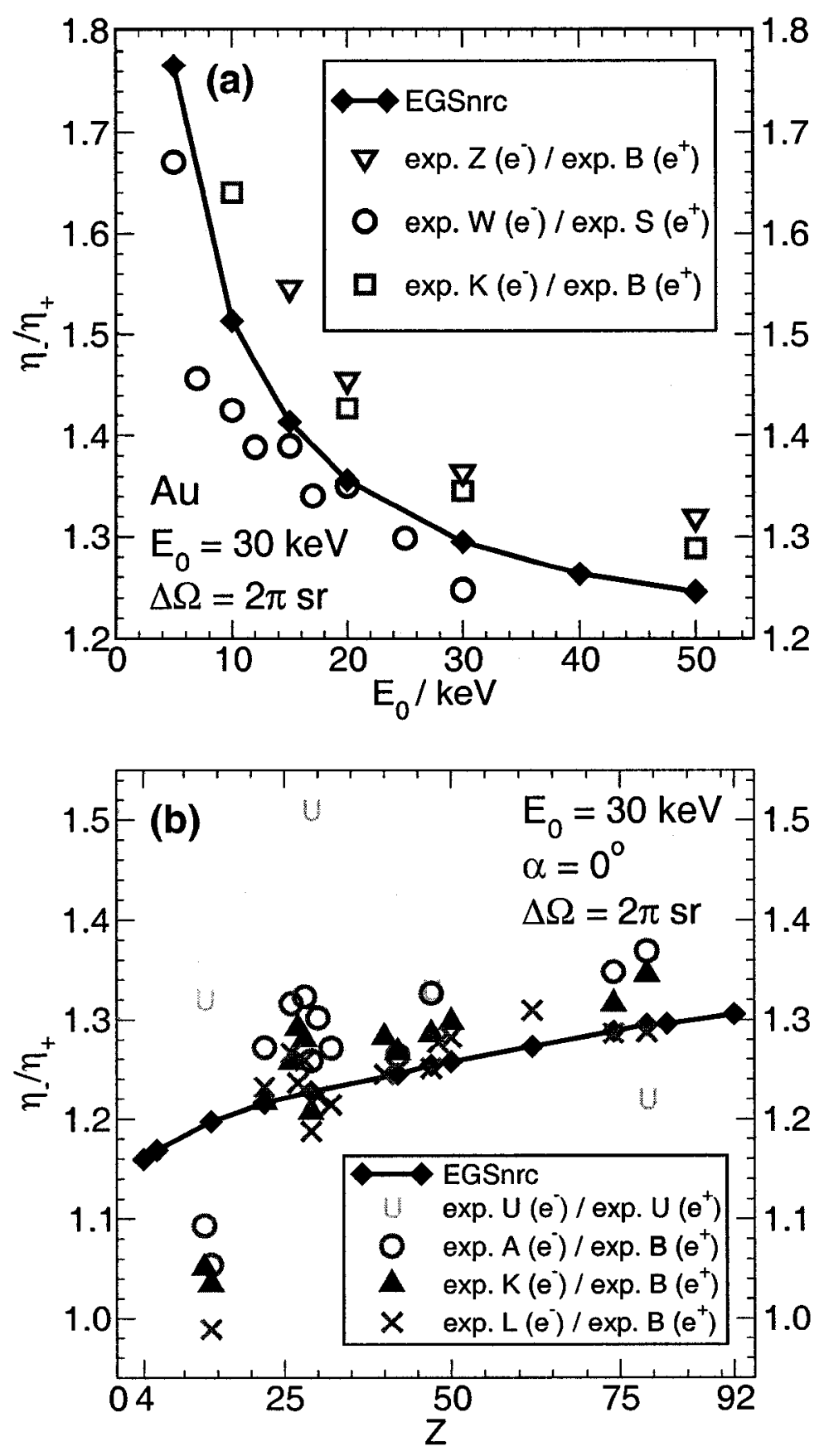

Figure 3.16: EGSnrc results versus experimental data for the variation of the ratio of the electron-to-positron total backscatter coefficient, $\eta_{-} / \eta_{+}$, with the incident charged particle energy, $E_{0}$ - panel $a$, and with the atomic number of the target material, Z - panel b. EGSnrc results (filled diamonds) are connected by solid line segments. In panel $b$, the ratio for $\mathrm{Be}(1.75 \pm 0.64)$ from experiment $U$ is not plotted for clarity of other data points, and also because it is reported with $40 \%$ uncertainty. Also in panel b, experiment $\mathrm{U}$ is at $35 \mathrm{keV}$, not $30 \mathrm{keV}$. 

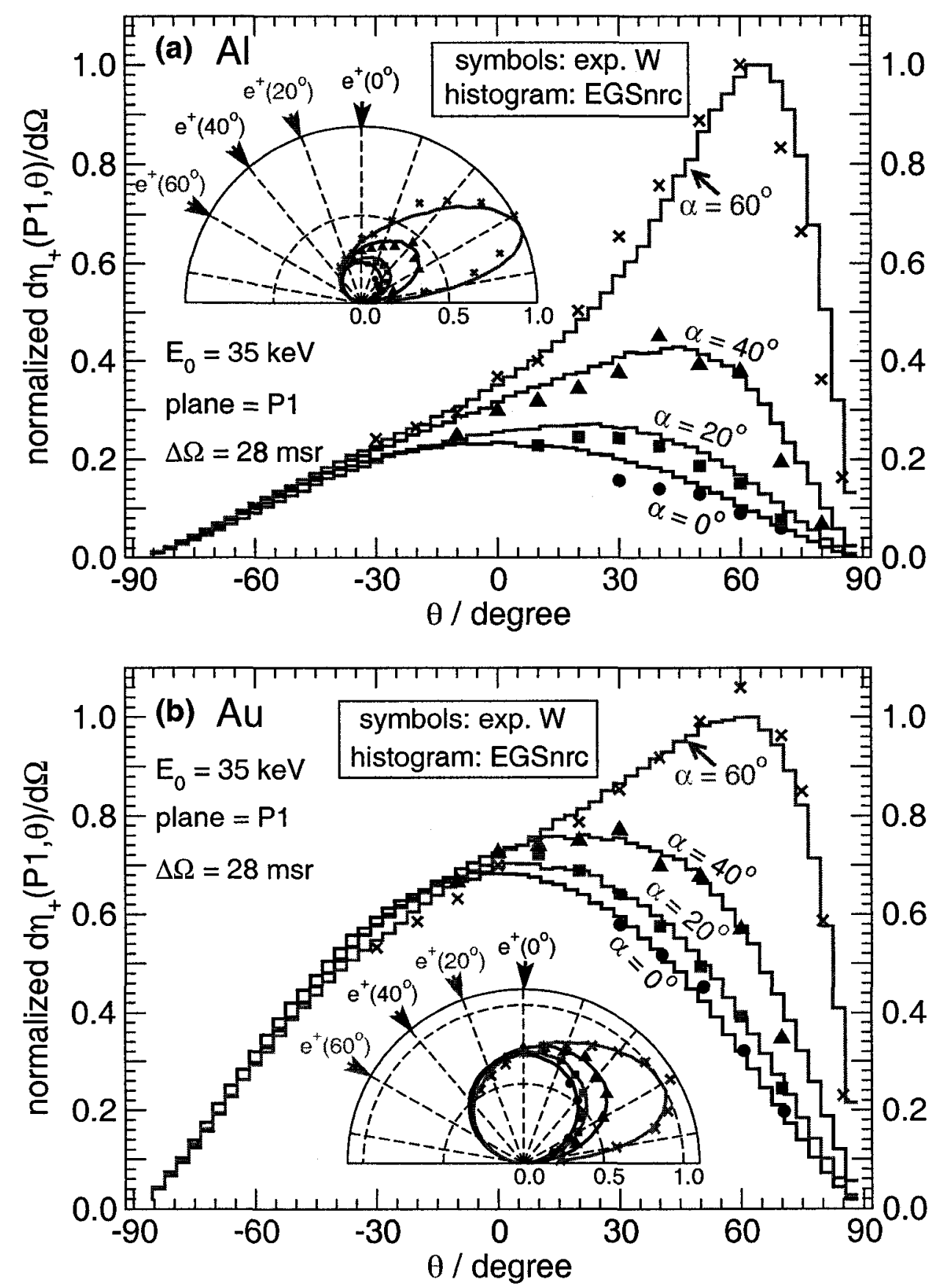

Figure 3.17: EGSnrc results versus experimental data for the variation of the angular distribution of backscattered positrons with the angle of incidence, $\alpha$, for $\mathrm{Al}$ and $\mathrm{Au}$. Experimental data are taken from experiment W. Normalization in panel b is arbitrary (i.e. not largest-peak to largest-peak), but the relative magnitude of all data points is preserved. 


\subsection{Summary}

This part of the project benchmarked the EGSnrc system in the energy range of interest to kilovoltage medical physics applications (5-140 keV). Given the underlying measurement uncertainties, and the fact that the EGSnrc results show excellent agreement with most experimental data, it can be concluded that the EGSnrc system produces accurate results in the kilovoltage range, and that the system is suitable for kilovoltage medical physics applications. In addition, the EGSnrc system can also be used to generate catalogues of backscatter data that are needed in many applied physics fields. An EGSnrc macro customized for backscatter calculations is available from the author upon request. More details on the material presented in this chapter can be found in Ali and Rogers. ${ }^{2,3}$ 


\section{Chapter 4}

\section{Off-focal radiation (OFR)}

\subsection{Introduction}

In an x-ray tube, when electrons impinge onto the target, a large fraction of them backscatter out of the target into the tube vacuum as discussed in chapter 3 . Because of the electric field applied between the cathode and the anode (the $\mathrm{kV}$ ), these backscattered electrons decelerate until they come to a stop, then they accelerate back towards the anode and re-enter it, mostly outside the focal spot. This causes what is known as the off-focal, or extra-focal, radiation (OFR) (figure 4.1). Backscattered electrons which re-enter the target have energies and angles of entry different from those of the original incident beam. Consequently, they alter the overall output of the x-ray tube. The presence of an off-focal component in the overall output of an $x$-ray tube has been known since the early days of $x$-ray tubes. $^{78}$ It is interesting to note that the first issue in the first volume of the Medical Physics journal has an article discussing OFR. ${ }^{79}$ Researchers have ruled out the possibility that the OFR component is mainly caused by mis-focused primary electrons ${ }^{80,81}$ (due to electric field distortions), or by scatter from peripheral

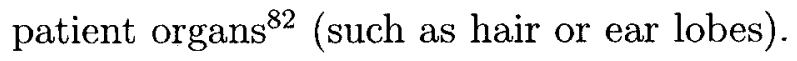




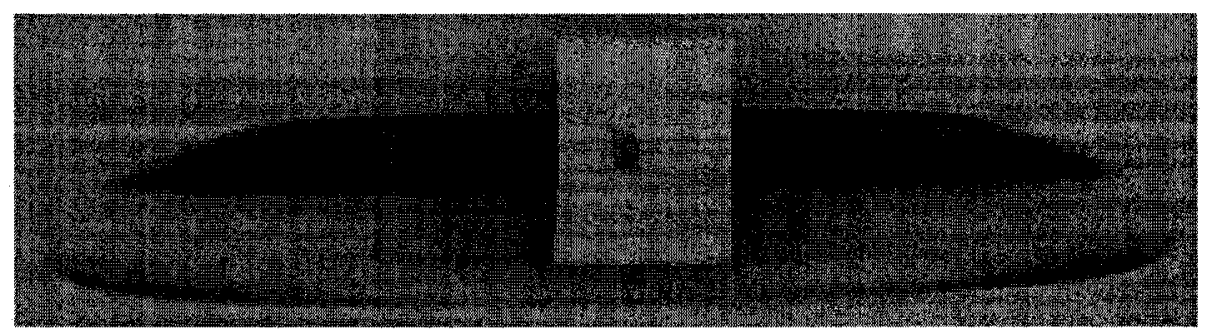

Figure 4.1: Example of OFR. The figure is an anode image at the SSD with the focal spot blocked. The dark parts of the anode that show in the image are due to OFR. Used with permission from Professor Paul Johns at Carleton University.

There are two distinct uses of the term "off-focal radiation (OFR)" in the literature. The first usage is to refer to $\mathrm{x}$ rays emanating only from outside the focal spot, regardless of their source, and regardless of where they hit the image receptor at the SSD. According to this usage, $\mathrm{x}$ rays generated by electrons which backscattered then re-entered the anode within the focal spot are considered part of the primary component. The second usage is to refer to features in the image receptor at the SSD that are outside the useful imaging field. According to this usage, $x$ rays within the useful imaging field at the SSD are considered primary, even if they originate from outside the focal spot. This part of the project adopts the first usage, i.e. neglecting mis-focused electrons, OFR from hereon refers to x-rays produced by backscattered electrons which re-enter the anode outside the focal spot. As will be seen in section 4.4, the fraction of backscattered electrons which re-enter the anode within the focal spot is negligible, and the usage of the term OFR can be relaxed to include all $x$-rays generated by backscattered electrons which re-enter the anode. This implies that at the SSD, OFR can have a component inside and a component outside the useful imaging field.

There is a large variation between investigators in quantifying the effect of the OFR component on $\mathrm{x}$-ray tube output. Different investigators reported OFR contribution to the overall exposure (or air kerma) at the patient plane, that

\subsection{INTRODUCTION}


ranges between 5 and $25 \% .{ }^{82-90}$ One investigator ${ }^{82}$ reported a contribution that varied from 5 to $22 \%$ for the same tube assembly, depending on the method of measurement. It is reported that no successful attempt has been made to standardize either the measurement method or the permissible levels of OFR in diagnostic machines. ${ }^{81}$

The contribution of OFR to the overall tube output depends on the design and operational parameters of the tube assembly. OFR is more pronounced for rotating anode targets because their disks are larger than stationary ones; it is also because stationary targets have copper surrounding the target (which has a low bremsstrahlung cross section), and so backscattered electrons that miss the tungsten target do not generate much OFR elsewhere. For the rest of this part of the project, only rotating anode targets are investigated, but the treatment is applicable to stationary targets as well. The amount of OFR strongly depends on the grounding configuration of the tube assembly. There are a variety of reasons for the choice of a particular type of grounding. ${ }^{91,92}$ It is reported that OFR in grounded cathode assemblies is a factor of 10 more than it is in grounded anode ones. $^{91}$

The OFR component is undesirable in x-ray systems. Its negative effects are similar to those of the scatter component. It impairs image quality, increases patient dose, and introduces errors in beam quality specification. ${ }^{86}$ The OFR component outside the useful imaging field shows on radiographs as faint images of anatomical structures outside the collimated field of interest (called penumbral images).$^{88}$ Other image artifacts include patches or concentric rings. ${ }^{81}$ OFR also introduces uncertainties in the determination of the total filtration of $\mathrm{x}$-ray tube assemblies (which is necessary for quality assurance). ${ }^{93,94}$ In the language of diagnostic radiology, OFR reduces the Modulation Transfer Function (MTF)

\subsection{INTRODUCTION}


at low frequencies, which implies that OFR is more of an issue for imaging larger structures than it is for finer details. ${ }^{83}$ OFR is also one of the reasons for the discrepancies reported in the literature in measurements and calculations of the Back-Scatter Factor (BSF) for kilovoltage dosimetry purposes. ${ }^{95}$ Very recently, OFR has been shown to strongly affect the output of x-ray tubes when placed in the magnetic field of an MR magnet. ${ }^{48}$ It is argued, however, that the effect of the scatter component on degrading the image quality is much stronger than that of the OFR component, which renders the OFR component insignificant in practice. ${ }^{82}$ Nevertheless, the same investigator ${ }^{82}$ confirms the significance of the OFR component in fluoroscopy systems with image intensifiers.

Reducing the OFR component is challenging because OFR is generated inside the vacuum envelope of $\mathrm{x}$-ray tube. A few of the methods used to reduce (but never eliminate) OFR are: ${ }^{82,91,96,97}$ Use of well-designed collimators right outside the tube assembly, use of filtration, careful arrangement of the elements inside the x-ray tube, placing diaphragms inside the x-ray tube very close to the focal spot, specific shaping of the anode surface and use of hooded anodes (for stationary targets only).

Because OFR is an inherent part of the output of $\mathrm{x}$-ray tubes, realistic simulation of these tubes requires including the OFR component. Only very few theoretical or computational attempts to describe or quantify the OFR effects have been reported. ${ }^{47,83,92}$ To the author's knowledge, there has never been a Monte Carlo study of OFR. The goals of this part of the project are to add to BEAMnrc the ability to simulate OFR for more realistic simulations of kilovoltage $x$-ray systems, and to quantify the effect of OFR on the output parameters of $\mathrm{x}$-ray tubes.

4.1. INTRODUCTION 


\subsection{Implementing OFR in BEAMnrc}

To add to BEAMnrc the ability to simulate OFR, the code is modified to transport electrons in an electric field in vacuum. The transport within the target is not altered because the electric field is zero inside a conductor. EGSnrc inherited from EGS4 a few macros for transporting charged particles in electromagnetic fields. These macros are not used here because they need to be adapted to work with EGSnrc (as opposed to EGS4), and also because the problem at hand is straightforward (transport is in vacuum not in media) and the macros are too general. It should also be noted that other major Monte Carlo codes used in medical physics research (Geant $4^{98}$ and PENELOPE ${ }^{36}$ ) can perform charged particle transport in electromagnetic fields in media.

The following assumptions are made in the implementation. (1) Primary electrons hit the anode normally. Finite element simulations of the electric field distribution in grounded cathode tube assemblies show that electrons hit the anode normally or near-normally. ${ }^{91,99}$ In addition, new tube designs have the cathode tilted to be parallel to the focal spot. ${ }^{100}$ Finally, from figure 3.5 page 57 , the variation of the total backscatter coefficient versus the incidence angle is very small for near-normal incidence $\left(\alpha \sim 0^{\circ}\right)$, and so ignoring possible small deviations should not affect the results. (2) Electric field distortions are ignored; the field is assumed uniform, constant, and perpendicular to the anode surface. (3) Possible radiation damping in vacuum due to acceleration and deceleration of backscattered electrons is ignored. (4) Although backscattered electrons can re-enter different parts of the target assembly, only those electrons which re-enter through the target face containing the focal spot are considered. This is because geometry constraints make it hard for other electrons to generate $\mathrm{x}$ rays that emerge from

\subsection{IMPLEMENTING OFR IN BEAMNRC}


the tube and reach the patient plane. (5) Because BEAMnrc does not have a component module suitable for simulating the full details of a rotating anode, an approximate rectangular target is used to simulate the portion of the anode that significantly contributes to the OFR. The OFR generated beyond the real round edges and up to the simulated rectangular edges is expected to be negligible.

The implementation takes into account that fact that electrons can backscatter multiple times. The convention in the implementation is that when an electron backscatters for the first time, it belongs to the first generation; if it backscatters again, it belongs to the second generation, but its original contribution to the first generation is kept. Backscattering more than twice generates multiple generations of backscattered electrons. The user is given the choice to include first, second or all generations of backscatter in the simulation.

Appendix A.1 presents the derivation of the equation for the range or lateral displacement, $R$, of the backscattered electrons in vacuum. Appendix A.2 shows the algorithm newly added to BEAMnrc to transport the backscattered electrons to their re-entry locations, and to determine their energies and their directions of re-entry. The treatment is relativistic because a non-relativistic treatment introduces errors of up to $20 \%$ in the calculations of the lateral displacement, $R$, in equation A.16 page 108 at orthovoltage energies.

\subsection{Benchmarking OFR in BEAMnrc}

Benchmarking the new addition of OFR in BEAMnrc is difficult. The useful experimental data available for quantitative comparisons are very scarce because many experiments only describe OFR effects qualitatively on radiographs. Most of the data are $3-4$ decades old, and most of the experiments are not described

\subsection{BENCHMARKING OFR IN BEAMNRC}


well enough to be simulated. The only available data to benchmark the results of higher generations of backscatter are from a theoretical model with simplifying assumptions. ${ }^{92,101}$ However, three experiments ${ }^{85,86,90}$ have good potential for possible meaningful comparisons.

\subsection{Results}

To understand and quantify the effect of the OFR on the output parameters of an x-ray tube at the patient plane, one needs to first analyze the source of OFR radiation at the $\mathrm{x}$-ray target. Simulation results of the characteristics of first and higher generations of backscattered electrons are presented first, followed by results of the overall effect of OFR on the tube output.

\subsubsection{First-generation backscatter}

BEAMnrc simulations show that the extent of spread of the backscattered electrons when they re-enter the anode strongly affects the OFR component at the patient plane. Equation A.16 page 108 shows that the range (or lateral displacement), $R$, of the backscattered electrons is inversely proportional to the electric field strength $E=k V p / d$, where $d$ is the cathode-anode separation (which ranges between 0.75 and $4.5 \mathrm{~cm}$ in typical x-ray tubes). This means that the lateral spread of the backscattered electrons is directly proportional to the cathode-anode separation, $d$. Because of geometric constraints, the larger the spread, the less chance that the OFR emanating from these electrons reaches the patient plane.

Figure 4.2 shows the effect of $d$ on the lateral displacement of backscattered electrons in a realistic tube. The figure shows that for $\mathrm{kVp}=65$ and $\mathrm{d}=0.75 \mathrm{~cm}$, all backscattered electrons land back on the target within $1.4 \mathrm{~cm}$ from their point of backscatter (which is typically very close to their point of impact when they first

\subsection{RESULTS}




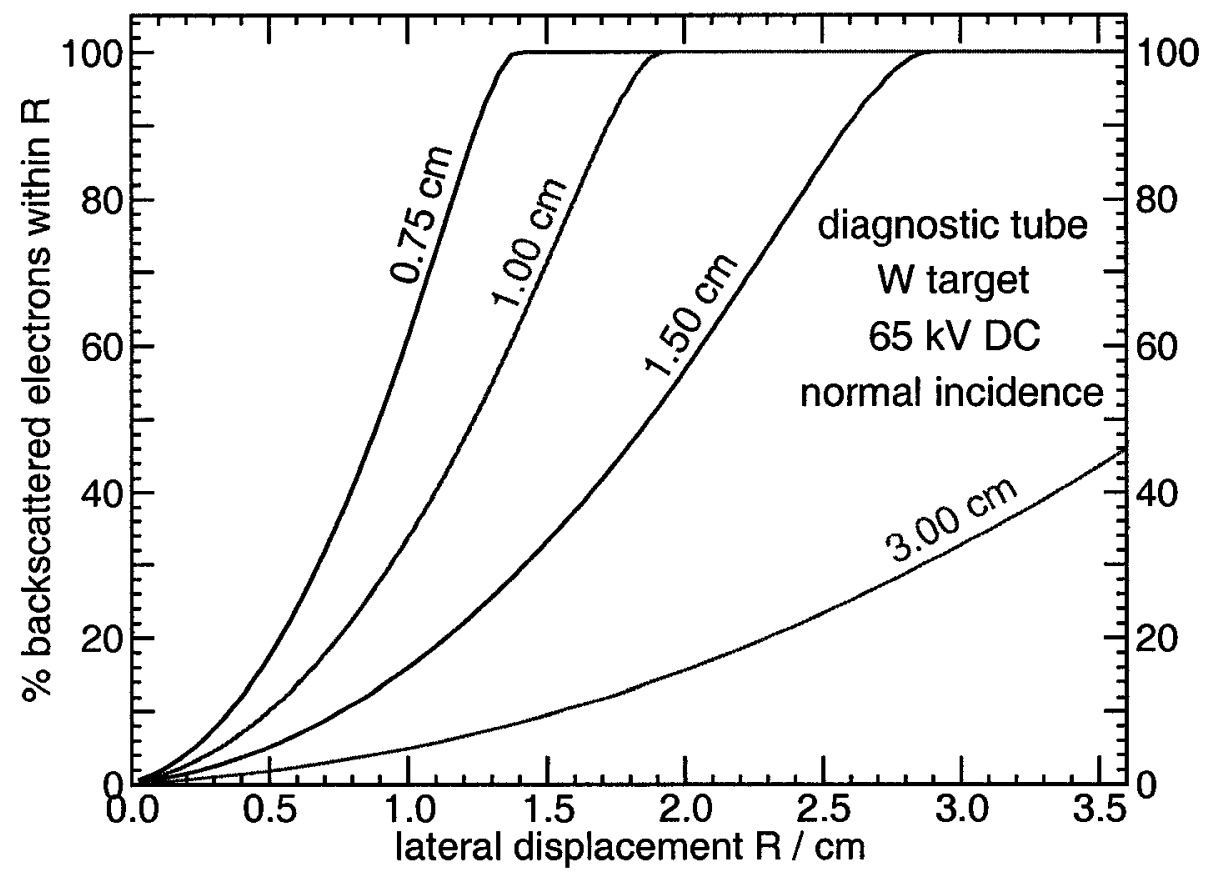

Figure 4.2: Variation of the lateral displacement of first generation backscattered electrons with the cathode-anode separation (labeled on each curve) for a given $k \vee p$.

hit the anode as primaries, particularly for high-Z targets). When $d$ is increased to $3 \mathrm{~cm}$ with the same $\mathrm{kVp}$, less than $50 \%$ of the backscattered electrons land within $3.5 \mathrm{~cm}$ of their backscatter position. The value of $d=1.5 \mathrm{~cm}$ is typical for $\mathrm{x}$-ray tubes (and it is the value measured from a demo glass insert in the x-ray lab at Carleton University). This value of $d=1.5 \mathrm{~cm}$ is used for the rest of this part of the project. A different choice of $d$ may strongly affect the results. Two other observations are worth mentioning about equation A.16. Because both total energy, $\varepsilon_{0}$, and electric field strength, $E$, depend on the $\mathrm{kVp}$, the lateral displacement, $R$, in the equation is not sensitive to the $\mathrm{kVp}$ for a given fractional energy of a backscattered electron. This means that for mammography, diagnostic and orthovoltage tubes, the spread of backscattered electrons is almost the same. Finally, it can be shown that when electrons backscatter at an angle near $45^{\circ}$ (not exactly $45^{\circ}$ as in non-relativistic treatment of charged particle motion), they undergo the farthest lateral displacement. 


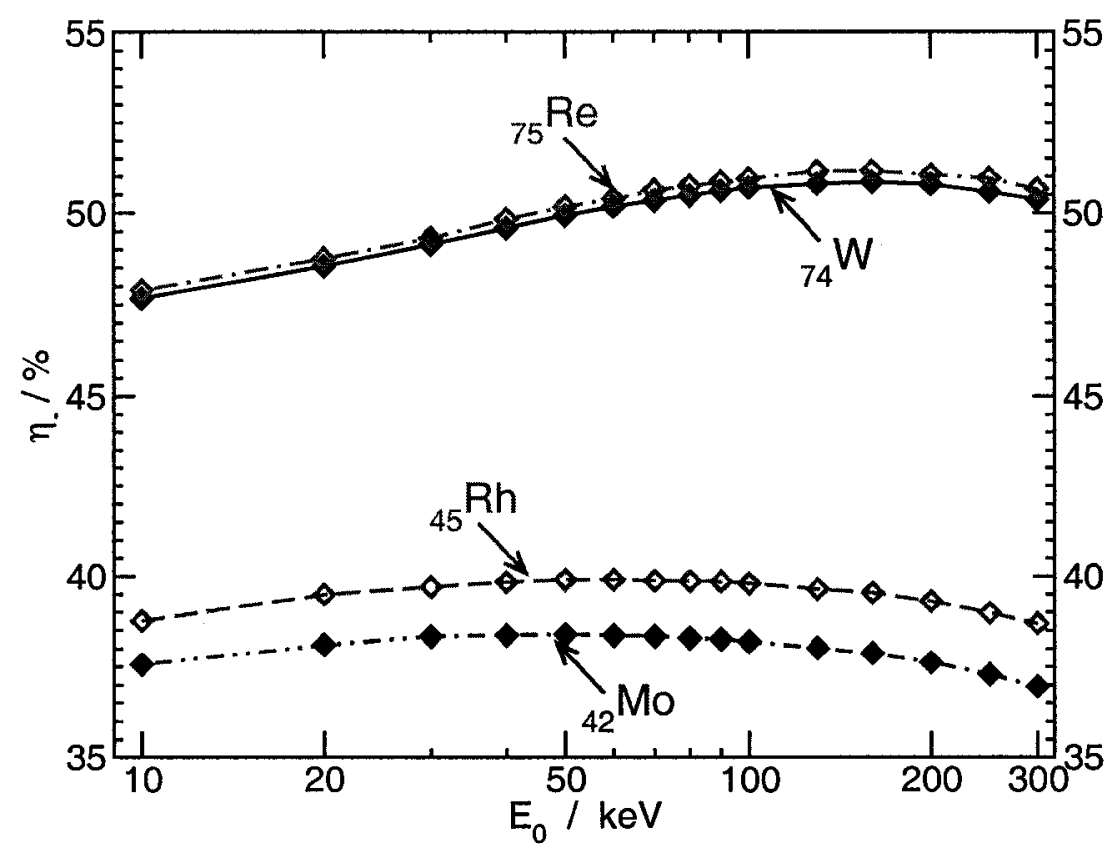

Figure 4.3: EGSnrc results for the first generation backscatter coefficient, $\eta_{-}$, for typical $x$-ray target elements in the energy range of interest in mammography, diagnostic and orthovoltage applications. $E_{0}$ is the kinetic energy of the incident monoenergetic beam. The scale of the abscissa is logarithmic.

After the exhaustive benchmark tests presented in chapter 3, EGSnrc can confidently be used to extract the characteristics of backscattered electrons. Figure 4.3 shows EGSnrc results of the backscatter coefficient, $\eta_{-}$, for the materials commonly used in x-ray target alloys. The reader is referred to section 3.5.1 for a full discussion. Similarly, figure 4.4 shows EGSnrc results for the energy spectra and angular distribution of first generation backscattered electrons for typical situations of $x$-ray tubes in mammography, diagnostic and orthovoltage applications. The reader is referred to sections 3.5 .2 and 3.5 .3 for a full discussion. For most medical x-ray tubes, about $\sim 50 \%$ of the incident electrons scatter back into the vacuum of the tube ( $\sim 38 \%$ in mammography). In all cases, backscattered electrons undergo almost the same lateral spread; they retain $\sim 90 \%$ of their original energy; a large fraction of them backscatter at an angle of $45^{\circ}$ and they are the ones that spread the farthest.

4.4. RESULTS 

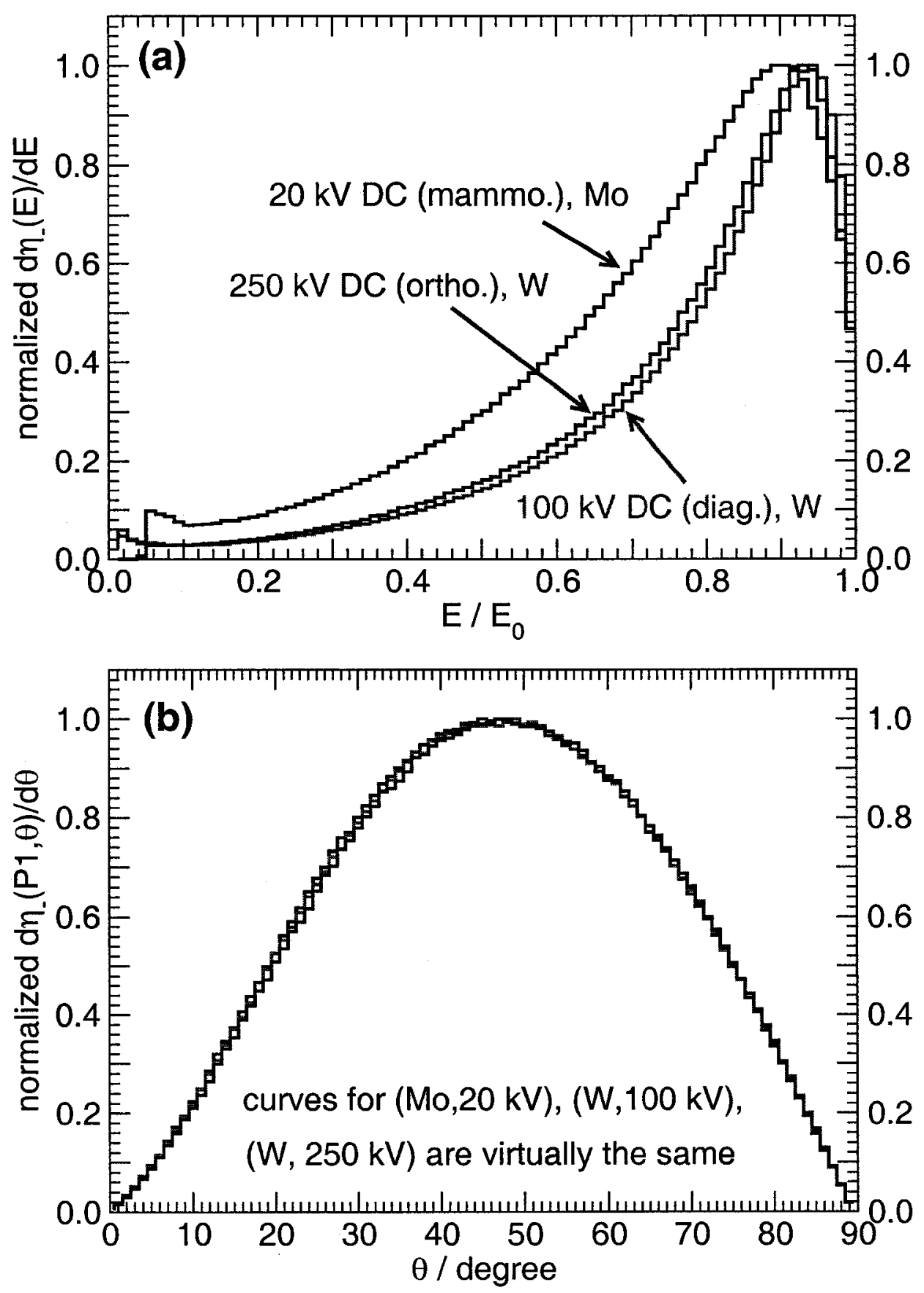

Figure 4.4: EGSnrc results for the energy spectra (panel a) and angular distribution (panel b) of first generation backscattered electrons for typical situations of $x$-ray tube usage (normal incidence). Unlike angular distribution graphs in chapter 3 , the distribution in panel $\mathrm{b}$ is per unit angle, $\theta$ (as opposed to per unit solid angle, $\Omega$ ). All curves are arbitrarily normalized to unity at their peak. 


\subsubsection{Higher generation backscatter}

Electrons that backscatter more than once are expected to have much less impact on the extent of OFR in an x-ray tube. This is because they are less in number and lower in energy (which means their bremsstrahlung cross section is smaller). Because the backscatter coefficient is almost energy independent in the kilovoltage range (see figure 3.3 page 54 ), when a fraction $f$ backscatters for the first time, $f$ of it will backscatter a second time, and so on. This is generally true, but two other factors come into play. The first is that backscattered electrons reenter the target at angles different from the angle of incidence of the primary incident beam, which alters the fraction backscattered from one generation to the next. The second is that the backscatter coefficient starts showing stronger energy dependence at lower energies. This implies that the backscatter between successive generations is expected to loosely follow a geometric series [which has a sum of $f /(1-f)]$. Table 4.1 corroborates this expectation. As an aside, the table also shows that the first generation backscatter is almost entirely due to primary electrons, not knock-on (secondary) electrons.

Figure 4.5 shows that the higher the backscatter generation, the more uniform their spread is. This can be attributed to the smearing of energies and angles from one generation of backscattered electrons to the next. The figure also shows that despite the difference in the average energy between backscatter generations, they spread to almost the same extent. This has been explained in section 4.4.1. Figure 4.6 shows that the energy spectrum dramatically changes towards lower energies from one generation to the next, while the angular distribution preserves its shape with some shift towards shallower backscatter angles.

4.4. RESULTS 
Table 4.1: Results using the modified BEAMnrc code for the multiple-generation backscatter coefficient (in percent) for typical situations of $x$-ray tube usage, assuming a cathode-anode distance of $1.5 \mathrm{~cm}$. The first generation backscatter coefficient is broken into two components: contribution from primary electrons and contribution from knock-on (secondary) electrons. For second and higher generation backscatter, a rectangular target of dimensions $10 \mathrm{~cm} \times 2.6 \mathrm{~cm}$ is used to represent the area of the anode (on the plane of the focal spot) which contributes to the OFR. Backscattered electrons that land outside this representative target area do not contribute to the numbers below for second and higher generations of backscatter.

\begin{tabular}{|c||c|c|c||c|c||c|}
\hline \multicolumn{1}{|c||}{ simulation case } & \multicolumn{3}{|c||}{ 1st generation } & 2nd & \multicolumn{1}{|c||}{$>$ 2nd } & total \\
& pri & sec & tot & tot & tot & \\
\hline \multirow{2}{*}{ mammography, Mo, 20 kV DC } & 36.71 & 0.88 & 37.59 & 11.58 & 6.26 & 55.43 \\
diagnostic, W, $100 \mathrm{kV} \mathrm{DC}$ & 49.43 & 1.12 & 50.55 & 18.14 & 13.76 & 82.45 \\
orthovoltage, W, $250 \mathrm{kV} \mathrm{DC}$ & 49.47 & 1.10 & 50.57 & 19.07 & 15.66 & 85.30 \\
& & & & & & \\
\hline
\end{tabular}

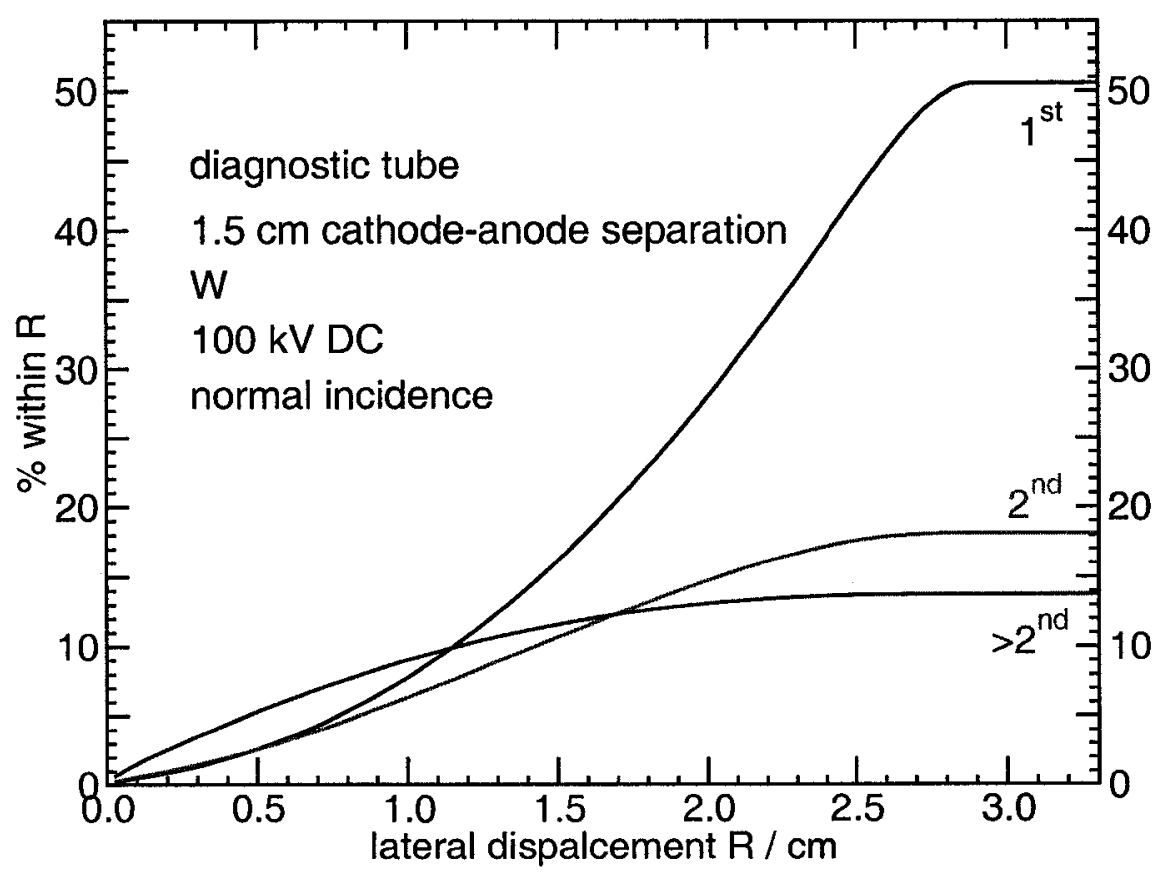

Figure 4.5: Results using the modified BEAMnrc code for the lateral displacement of different generations of backscattered electrons for the diagnostic tube in table 4.1. Consult the caption of table 4.1 for more details. Saturation values for each curve are those reported in table 4.1. 

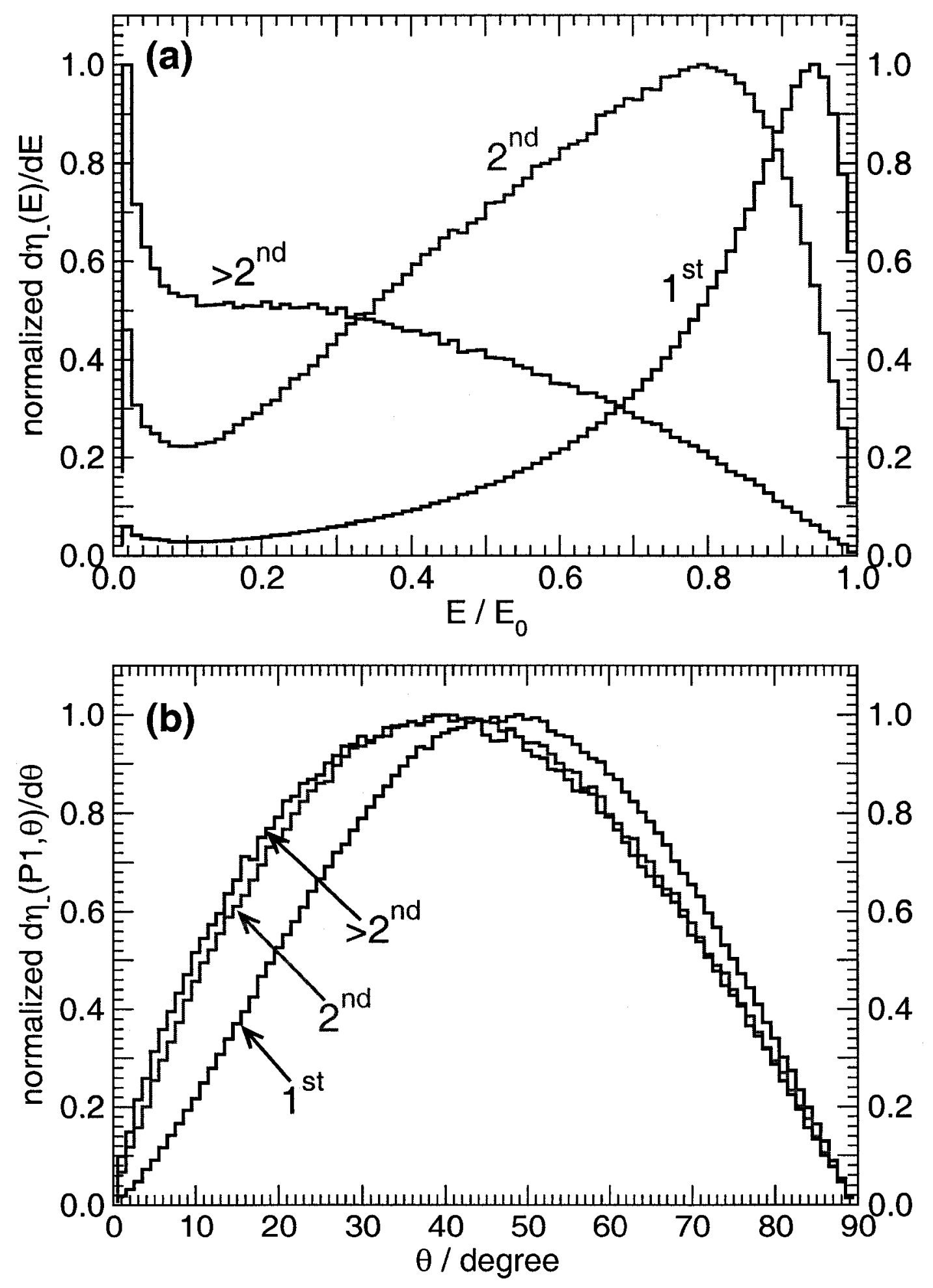

Figure 4.6: Results using the modified BEAMnrc code for the energy spectra (panel a) and angular distribution (panel b) of multiple-generation backscattered electrons for typical situations of $x$-ray tube usage. The distribution in panel $b$ is per unit angle, $\theta$. All curves are arbitrarily normalized to unity at their peak.

4.4. RESULTS 


\subsubsection{Effect of OFR on output parameters of x-ray tubes}

In this section, typical x-ray tube assemblies are simulated, and the effect of the OFR component on the output parameters at the patient plane is studied. The simulated diagnostic tube has a $16^{\circ}$ target $(90 \% \mathrm{~W}$ and $10 \% \mathrm{Re})$ with dimensions equivalent to the portion of the anode that contributes to the OFR $(10 \mathrm{~cm} \mathrm{x}$ $2.6 \mathrm{~cm}$ ). The filtration used is $0.5 \mathrm{~mm}$ aluminum equivalent inherent filtration, followed by $2 \mathrm{~mm}$ aluminum added filtration. Manufacturer's collimators $(2 \mathrm{~mm}$ thick lead plates) are placed immediately outside the exit window, where it creates a maximum field of $56 \times 56 \mathrm{~cm}^{2}$ at $100 \mathrm{~cm}$ SSD. Jaws to shape diagnostic fields are placed at $15 \mathrm{~cm}$. For the orthovoltage simulations, typical filtration $(0.5 \mathrm{~mm} \mathrm{Cu}$ followed by $2 \mathrm{~mm} \mathrm{Al}$ ) and typical applicators are used. ${ }^{19}$ Situations of no practical interest (such as a laterally-infinite target, or a tube without its manufacturer's collimation), are not included because they can lead to unrealistically-large OFR contribution. To estimate the effect of OFR on the HVL and the effective energy, BEAMnrc is used for the simulations to generate the spectra, then the newly developed user-code $h v l_{-} c l r p$ is used to determine the HVL and the effective energy (refer to section 1.3 for full details).

Figure 4.7 shows the effect of OFR on the output spectrum of an x-ray tube. Despite the poor statistics of the OFR component, it can be seen that it is softer ${ }^{86}$ than the primary spectrum, and that it causes a very slight shift ${ }^{89}$ in the overall spectrum. It also causes noticeable change (reduction in the case of high energy peaks) in the magnitude of the characteristic peaks. ${ }^{92,101}$

4.4. RESULTS 


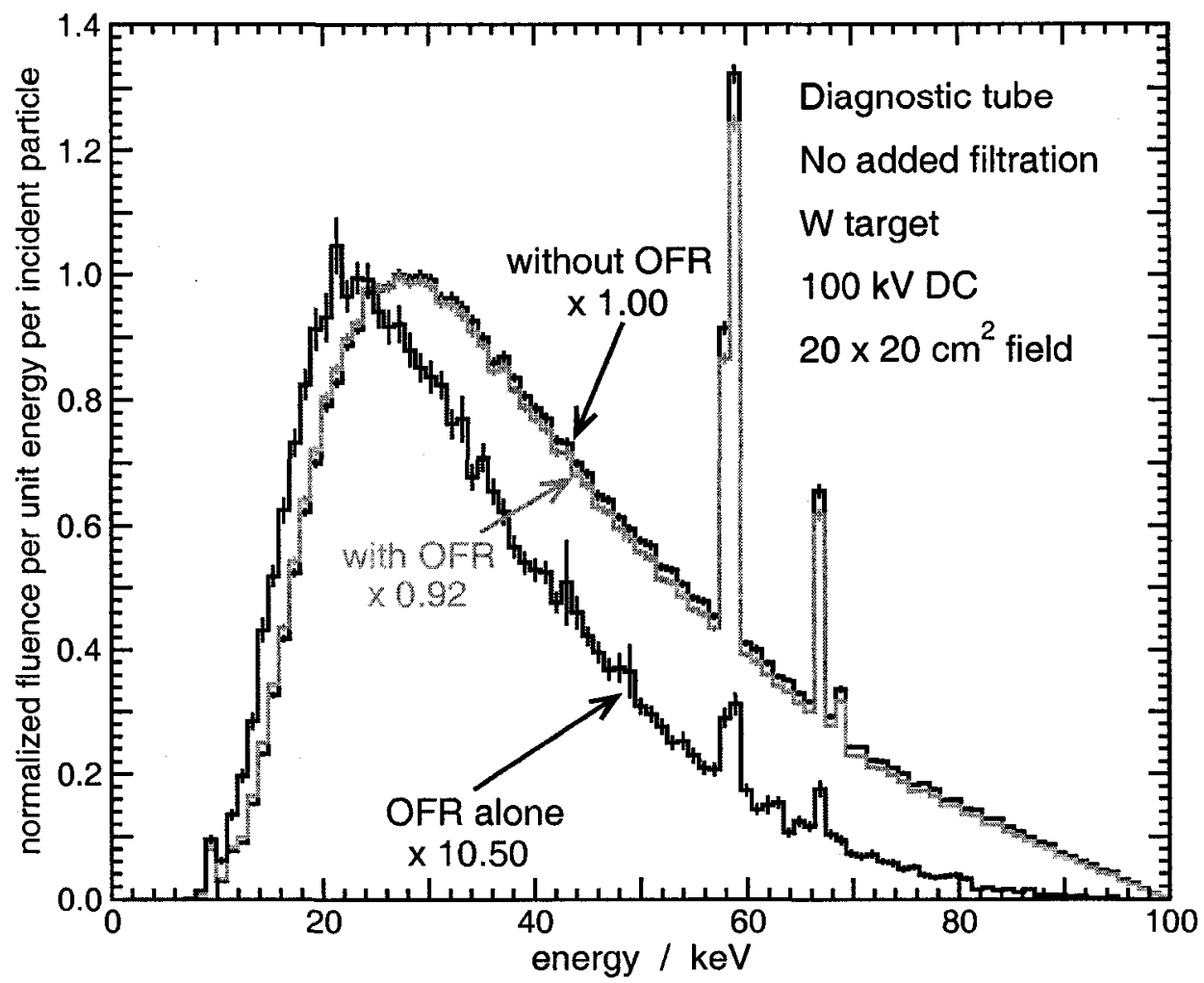

Figure 4.7: Results using the modified BEAMnrc code for the effect of OFR on the output spectrum of a diagnostic $x$-ray tube. The simulation parameters are: $16^{\circ} \mathrm{W}$ target, $100 \mathrm{kV}$ DC, $1.5 \mathrm{~mm}$ cathode-anode separation, manufacturer's collimation in place, no added filtration, and normal incidence. Spectra are averaged over a $20 \times 20 \mathrm{~cm}^{2}$ field at $100 \mathrm{~cm}$ SSD. All spectra are arbitrarily normalized to unity at the peak of the bremsstrahlung spectrum. OFR slightly softens the overall spectrum.

Figure 4.8 shows the effect of OFR on the tube output in terms of air kerma, HVL and effective energy for various $\mathrm{kVp}$. When only the manufacturer's collimation is used without added filtration (panel a), the contribution of the OFR can be significant (up to $10 \%$ for $K_{a i r}$ and HVL). The OFR contribution increases with increasing $\mathrm{kVp}$, which is contradicted by a few investigators, ${ }^{101}$ and supported by most others. ${ }^{83,86,97}$ The OFR component alone (panel b) is dramatically softer than the primary component. When typical filtration of diagnostic and orthovoltage systems is added (panel c), the OFR contribution gets smaller than it is without added filtration. With added filtration, the OFR contribution noticeably 

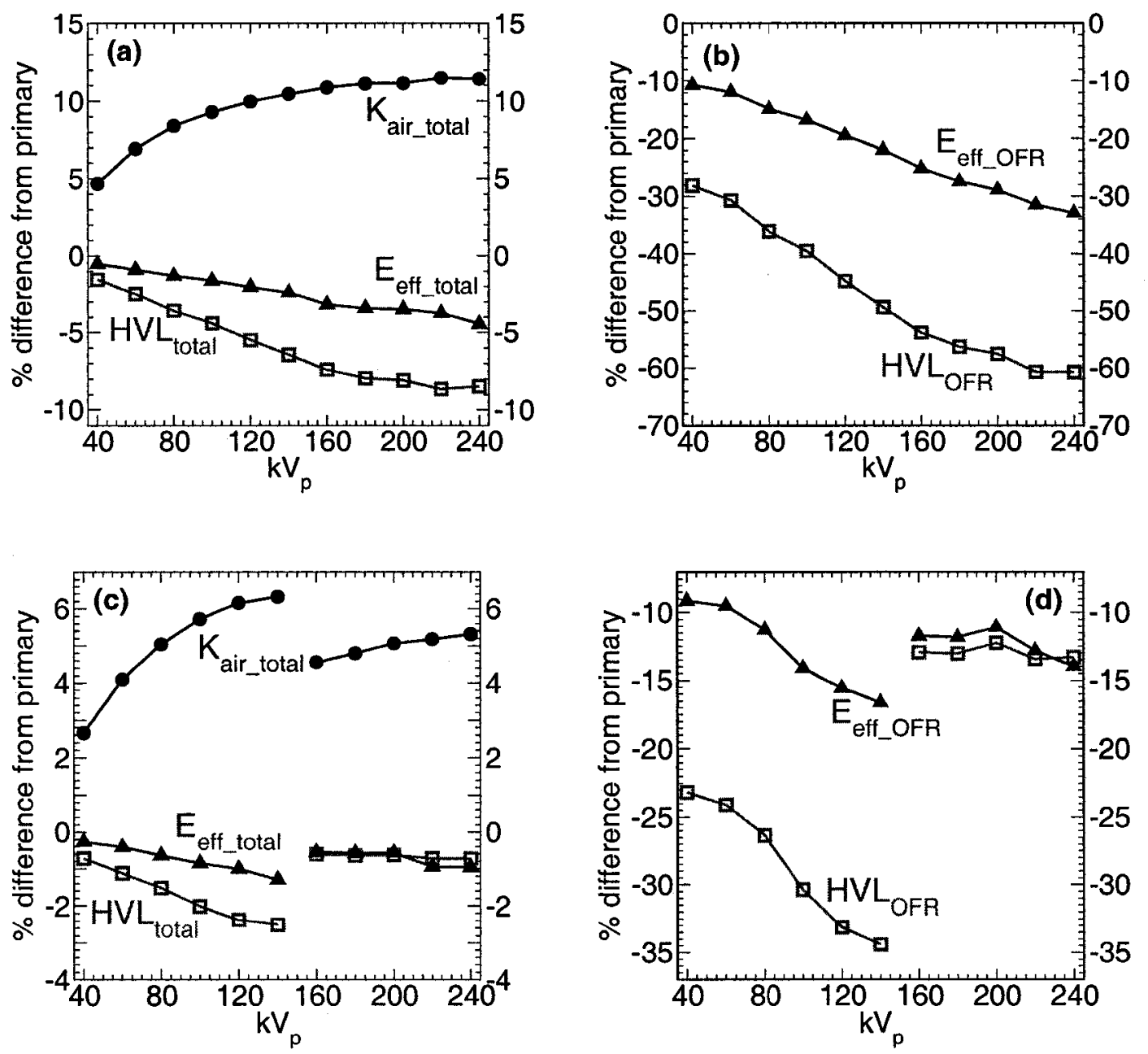

Figure 4.8: Modified BEAMnrc results for the effect of OFR on the tube output in terms of air kerma, HVL and effective energy, for various $k V p$. For all panels, the cathode-anode separation is $1.5 \mathrm{~cm}$. Panels a and $\mathrm{b}$ : manufacturer collimation is in place, no added filtration, and spectra are averaged over a $20 \times 20 \mathrm{~cm}^{2}$ field at $100 \mathrm{~cm}$ SSD. Panels $\mathrm{c}$ and $\mathrm{d}$ : manufacturer collimation is in place, added filtration of $2 \mathrm{~mm} \mathrm{Al}$ for $\mathrm{kV}_{p}<140$ and $0.5 \mathrm{~mm} \mathrm{Cu}+2 \mathrm{~mm} \mathrm{Al}$ for $\mathrm{kV}_{p}>140$ (in order for the effect to be as realistic as possible), and spectra are averaged over a $20 \times 20$ $\mathrm{cm}^{2}$ diagnostic field at $100 \mathrm{~cm} \mathrm{SSD} \mathrm{for} \mathrm{kV}_{p}<140$, and averaged over a $10 \times 10$ $\mathrm{cm}^{2}$ therapeutic field at $52 \mathrm{~cm} \mathrm{SSD} \mathrm{for} \mathrm{kV}_{p}>140$. Panels a and $\mathrm{c}$ : The subscript "total" represents the primary component + the OFR component. Panels b and $\mathrm{d}$ : The subscript "OFR" represents the OFR component alone. 
increases with $\mathrm{kVp}$ for the typical diagnostic tube, and slightly increases with $\mathrm{kVp}$ for the typical orthovoltage tube. For a typical $\mathrm{kVp}$ of 100 and $2 \mathrm{~mm} \mathrm{Al}$ filtration, OFR contributes $\sim 6 \%$ to the air kerma (and hence to the patient dose), and reduces the HVL of the primary spectrum by $\sim 2 \%$. The heavy filtration in the typical orthovoltage arrangement is the reason for the diminishing effect of the OFR.

Figure 4.9 shows the effect of OFR on the tube output in terms of air kerma, HVL and effective energy for various added filtrations typical of diagnostic and orthovoltage systems. The more the filtration, the less the contribution of the OFR component to all the output parameters at the patient plane. It is worth noting that Mainegra Hing and Kawrakow ${ }^{17}$ simulated the NRC DC x-ray tube and obtained agreement at the $2 \%$ level with the experimental HVLs without including the OFR contribution in their simulation. When their tube is re-simulated (by the author of this thesis) to include the OFR contribution, no noticeable effect on the HVL is observed. Further investigation shows that their use of heavy filtration for their HVL measurements completely suppresses possible OFR contribution, particularly at the high $\mathrm{kVp}$ range of their experiments. This can easily be seen in panel $\mathrm{c}$ for all filtration thicker than $2 \mathrm{~mm} \mathrm{Al}+0.25 \mathrm{~mm} \mathrm{Cu}$.

Finally, timing results show that including only the first generation of backscatter in a typical x-ray tube simulation increases the CPU simulation time by $\sim 30 \%$. First and second generation together increase the CPU time by $\sim 45 \%$, and including all backscatter generations causes an increase of $\sim 55 \%$. This is consistent with the expectations based on the amount of backscatter reported in table 4.1. Currently, the code has an option which allows the user to choose how many backscatter generations to include (the options are: one, two and all).

4.4. RESULTS 

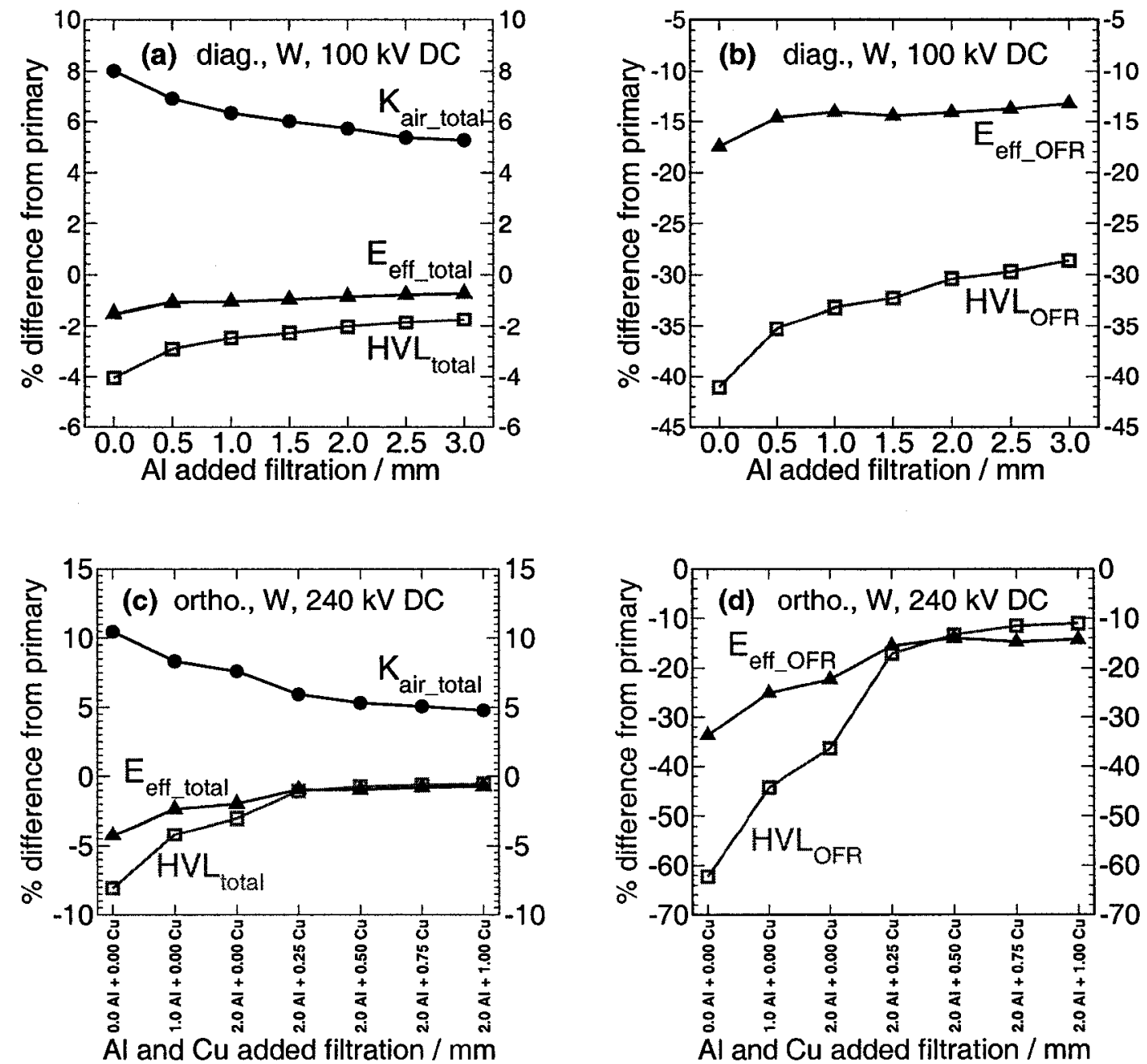

Figure 4.9: Results using the modified BEAMnrc code for the effect of OFR on the tube output in terms of air kerma, HVL and effective energy, for various added filtrations in a typical diagnostic tube (panels $a, b$ ), and a typical orthovoltage tube (panels $c, d$ ). Refer to the caption of figure 4.8 for details. 


\subsection{Summary}

This part of the project implements a new feature in BEAMnrc to simulate OFR in x-ray tubes. The extent of the OFR contribution depends on many parameters, such as the $\mathrm{kVp}$, the cathode-anode separation, and the details of collimation and filtration. This makes EGSnrc/BEAMnrc the first Monte Carlo code to include this feature, and it will allows researchers to simulate $\mathrm{x}$-ray tubes more realistically. 


\section{Chapter 5}

\section{Future work}

In addition to further investigation of the OFR, and continuing along the main theme of this project to make the EGSnrc/BEAMnrc system more efficient, accurate and realistic in simulating kilovoltage $\mathrm{x}$-ray systems, the following issues could be pursued:

- Anode roughness: as x-ray tubes age, their output changes due to increased roughness of the anode. ${ }^{102} \mathrm{~A}$ study to include anode roughness in BEAMnrc is needed for more realistic $\mathrm{x}$-ray tube simulation.

- Generalized transport in electromagnetic field: Generalization of charged particle transport to include transport in electric and magnetic fields would be a valuable addition. It gives flexibility to simulate situations such as the presence of an x-ray tube in the magnetic field of an MR magnet in hybrid CT/MRI systems, ${ }^{48}$ or the electromagnetic steering of the electron beams in recent designs of $\mathrm{x}$-ray tubes. 100

- Higher order atomic relaxations: For efficiency considerations, the EGSnrc system averages higher order atomic relaxations ${ }^{5}$ (e.g. M-L and N-L relaxations). Although this approach is accurate for most practical medical physics situations, it 
may not be suitable for certain benchmarks of spectra at very low energies. ${ }^{28}$ The option of explicit treatment of higher order atomic relaxations could be included in the system.

- Benchmarks of $\mathrm{x}$-ray spectra: There is a strong need for benchmarking the EGSnrc/BEAMnrc system against experimental x-ray spectra. The problem often encountered is that there are not enough specifications about the experiments and the about the detector spectral response correction that allow the Monte Carlo code to simulate their full details.

- Anti-scatter grid benchmarks: As part of this project, BEAMnrc is now capable of simulating anti-scatter grids, but the feature has not been benchmarked. The experimental measurements available in the literature for the performance of various grids can be used to benchmark this feature in BEAMnrc.

- Scatter-to-primary (S/P) benchmarks: There are plenty of experimental data measuring S/P for various diagnostic situations. Simulation of these situations is needed in order to benchmark the EGSnrc/BEAMnrc system for such applications, and also to answer questions related to S/P research that may otherwise be hard to answer.

- X-ray GUI: There is a need for a graphic user interface in the form of a standalone executable file dedicated to simulating x-ray tubes. The GUI should not require the user to have prior knowledge of Monte Carlo or the EGSnrc/BEAMnrc system. The input parameters the user is prompted to enter should be the ones that are directly related to the $\mathrm{x}$-ray system being simulated [target, voltage ripple, filtration and collimation, off-focal radiation, tube age (for anode roughness), ... etc]. This is expected to greatly expand the number of users who benefit from all the additions to the system for kilovoltage applications. 


\section{Chapter 6}

\section{Conclusions}

This project aims at making the EGSnrc/BEAMnrc system more efficient, accurate and realistic for kilovoltage medical physics applications in general, and for kilovoltage $\mathrm{x}$-ray systems in particular.

Chapter 2 describes the implementation and benchmarking of the Bremsstrahlung Cross Section Enhancement (BCSE) variance reduction technique in the EGSnrc/ BEAMnrc system. The technique scales up the rare event of bremsstrahlung production, and then re-works the simulation details to keep the results unbiased. When BCSE is combined with the existing splitting techniques in the system, efficiency gains in the simulations can be up to five orders-of-magnitude over those obtained with analog simulations, and up to a full order of magnitude over those without the new technique. This part of the project concludes that the BCSE technique should be combined with the existing variance reduction techniques in EGSnrc/BEAMnrc for both kilovoltage and megavoltage simulations that involve bremsstrahlung targets. Two easy optimization steps are also provided. 
Chapter 3 benchmarks the EGSnrc system in the energy range of interest to kilovoltage medical physics applications (5-140 keV). The benchmarks compare EGSnrc results against experimental data from 26 independent experiments. The agreement is excellent between EGSnrc results and most of the experimental data. There are many experimental uncertainties that could be reasons for the few noted discrepancies. This part of the project concludes that the EGSnrc system produces accurate results in the kilovoltage range, and that the system is suitable for kilovoltage medical physics applications. The EGSnrc system can also be used to generate catalogues of backscatter data that are needed in many applied physics fields.

Chapter 4 describes the implementation and benchmarking of the off-focal (extra-focal) radiation (OFR) in the BEAMnrc system. BEAMnrc is modified to include the transport of multiple generations of backscattered electrons in an electric field in vacuum. Results show that the OFR spectrum is softer, with effective energy 10-30\% lower than the main spectrum. Consequently, the HVL of the overall spectrum can be reduced by up to $10 \%$. The OFR component can also increase the air kerma at the patient plane by up to $10 \%$. This part of the project concludes that electrons backscattering from the targets of x-ray tubes play an important role in x-ray tube simulation. The OFR caused by these backscattered electrons has a non-negligible effect on the output parameters of x-ray tubes and it should be included in the simulations.

This project has contributed many new features to the EGSnrc/BEAMnrc system. The following could be a useful summary of these new features, including those that have not been discussed within the text of this thesis. 
- Bremsstrahlung cross section enhancement: A new variance reduction technique for use in simulations of bremsstrahlung targets, both in the kilovoltage and megavoltage range.

- Off-focal radiation: The ability to include off-focal radiation for more realistic simulation of $\mathrm{x}$-ray tubes.

- Backscatter macro: A new macro to simulate backscatter experiments and to report backscatter coefficient, energy and angular distribution of backscattered charged particles.

- hvl_clrp: A small user-code newly developed (in collaboration with D. La Russa) to calculate air kerma, HVL and effective energy given an energy fluence spectrum and an absorber material.

- Voltage ripple: The ability to include voltage ripple for more realistic simulation of $\mathrm{x}$-ray tubes (details not included in this thesis).

- Anti-scatter grids: BEAMnrc component module MLC is modified to be able to simulate parallel, focused and cross grids. (details not included in this thesis).

- Grid scoring The ability to score particles in a rectangular grid in the scoring plane. Previously, the only two options available were concentric circles and concentric squares (original macros kindly provided by B. Walters at NRC).

- Rejection plane: A rejection plane is added to be used with DBS. It prevents photons of large weight (fat photons) from interacting in the air layer just above the scoring plane and degrading the statistics of the scored quantities.

- Boundary tolerance: A new default value is proposed for the boundary tolerance parameter, \$BDY_TOL, in BEAMnrc for more accurate kilovoltage simulations. 


\section{Appendix A}

\section{OFR implementation}

\section{A.1 Relativistic kinematics of electron motion in vacuum in a uniform electric field}

In chapter 4 , to simulate the off-focal radiation in x-ray tubes, the equation of motion for an electron in a uniform electric field needs to be solved to determine the trajectory of the backscattered electrons in the vacuum in front of the target, which is then used to determine where they re-enter the target. In what follows, $E$ is the electric field strength (constant, uniform and perpendicular to the target surface), $c$ is the speed of light, $-e, m_{0}$ and $m$ are the electron charge, rest mass and total mass, respectively. At time $t$ after the instant of backscattering into the vacuum, the electron has a position vector $\vec{x}$, a velocity vector $\vec{v}$, a relative velocity vector $\vec{\beta}=\vec{v} / c$, a momentum vector $\vec{p}$, and a total energy $\varepsilon$. In the plane of motion, vector quantities can be split into a component parallel to the electric field $\left(x_{\|}, v_{\|}, \beta_{\|}, p_{\|}\right)$, and a component perpendicular to it $\left(x_{\perp}, v_{\perp}, \beta_{\perp}, p_{\perp}\right)$. This means that, by definition, the components of the electric field are: $E_{\|}=E$ and $E_{\perp}=0$. The instant of getting out of the target into the vacuum, and the instant 
of re-entering the target are denoted by the subscripts 0 and $f$, respectively. The goal is to obtain the range $R$ (can also be called the lateral displacement) of the electron at which $x_{\|}=0$ and $x_{\perp}=x_{\perp_{\max }}=R$, because this is where the electron re-enters the target. From the basic equations of momentum $(\vec{p}=m \vec{v})$ and total energy $\left(\varepsilon=m c^{2}\right)$, the velocity vector can be written as:

$$
\vec{v}=\vec{p} c^{2} / \varepsilon \text {. }
$$

Breaking equation A.1 into its parallel and perpendicular components,

$$
v_{\|}=d x_{\|} / d t=p_{\|} c^{2} / \varepsilon \quad \text { and } \quad v_{\perp}=d x_{\perp} / d t=p_{\perp} c^{2} / \varepsilon
$$

Expressions for $p_{\|}, p_{\perp}$ and $\varepsilon$ are needed to plug into equation A.2 and get the parametric equations of $x_{\|}$and $x_{\perp}$. This is obtained using the basic equation of motion of the backscattered electrons, given by

$$
\vec{p}=-e \vec{E},
$$

where $\vec{p}$ is the rate of change of momentum. Breaking equation A.3 into its parallel and perpendicular components,

$$
\dot{p}_{\|}=-e E \quad \text { and } \quad \dot{p}_{\perp}=0 .
$$

Integrating with respect to time,

$$
p_{\|}=-e E t+p_{\|_{0}} \quad \text { and } \quad p_{\perp}=p_{\perp_{0}} .
$$

The total energy is given by:

$$
\varepsilon=\sqrt{m_{0}^{2} c^{4}+p^{2} c^{2}} .
$$

In equation A.6, $p$ can be expressed (using equation A.5) as:

$$
\begin{aligned}
p^{2} & =p_{\|}^{2}+p_{\perp}^{2} \\
& =e^{2} E^{2} t^{2}-2 e E p_{\|_{0}} t+p_{\|_{0}}^{2}+p_{\perp_{0}}^{2} .
\end{aligned}
$$

Putting equation A.7 into equation A.6,

$$
\varepsilon=\sqrt{m_{0}^{2} c^{4}+e^{2} E^{2} c^{2} t^{2}-2 e E p_{\|_{0}} c^{2} t+p_{\|_{0}}^{2} c^{2}+p_{\perp_{0}}^{2} c^{2}} .
$$

\section{A.1. RELATIVISTIC KINEMATICS OF ELECTRON MOTION}


At $t=0$, equation A.8 reduces to:

$$
\varepsilon_{0}=\sqrt{m_{0}^{2} c^{4}+p_{\|_{0}}^{2} c^{2}+p_{\perp_{0}}^{2} c^{2}}
$$

Putting equation A.9 back into equation A.8,

$$
\varepsilon=\sqrt{\varepsilon_{0}^{2}+e^{2} E^{2} c^{2} t^{2}-2 e E p_{\|_{0}} c^{2} t}
$$

Putting equation A.5 and equation A.10 into equation A.2 and integrating with respect to time, we get the parametric equations as follows:

- For $x_{\|}$:

$$
\int_{0}^{x_{\|}} d x_{\|}^{\prime}=\int_{0}^{t} \frac{-e E c^{2} t^{\prime}+p_{\|_{0}} c^{2}}{\sqrt{\varepsilon_{0}^{2}+e^{2} E^{2} c^{2} t^{\prime 2}-2 e E p_{\|_{0}} c^{2} t^{\prime}}} d t^{\prime}
$$

Carrying out the integration,

$$
x_{\|}=\frac{1}{e E}\left[\varepsilon_{0}-\sqrt{\varepsilon_{0}^{2}+e^{2} E^{2} c^{2} t^{2}-2 e E p_{\|_{0}} c^{2} t}\right] .
$$

- For $x_{\perp}$ :

$$
\int_{0}^{x_{\perp}} d x_{\perp}^{\prime}=\int_{0}^{t} \frac{p_{\perp_{0}} c^{2}}{\sqrt{\varepsilon_{0}^{2}+e^{2} E^{2} c^{2} t^{\prime 2}-2 e E p_{\|_{0}} c^{2} t^{\prime}}} d t^{\prime}
$$

Carrying out the integration (by completing the square under the root sign),

$$
x_{\perp}=\frac{p_{\perp_{0}} c}{e E}\left[\sinh ^{-1}\left(\frac{p_{\|_{0}} c}{\sqrt{\varepsilon_{0}^{2}-p_{\|_{0}}^{2} c^{2}}}\right)-\sinh ^{-1}\left(\frac{p_{\|_{0}} c-e E c t}{\sqrt{\varepsilon_{0}^{2}-p_{\|_{0}}^{2} c^{2}}}\right)\right]
$$

Equations A.12 and A.14 are the parametric equations. The backscattered electron re-enters the target when $x_{\|}=0, t=t_{f}$ and $x_{\perp}=x_{\perp_{\max }}=R$.

Putting $x_{\|}=0$ in equation A.12 gives $t_{f}=2 p_{\|_{0}} / e E$. Putting $t_{f}$ into equation A.14 gives the range $R$,

$$
R=x_{\perp_{m a x}}=\frac{2 p_{\perp_{0}} c}{e E} \sinh ^{-1}\left(\frac{p_{\|_{0}} c}{\sqrt{\varepsilon_{0}^{2}-p_{\|_{0}}^{2} c^{2}}}\right)
$$

\section{A.1. RELATIVISTIC KINEMATICS OF ELECTRON MOTION}


Using $p_{\|_{0}} c=\varepsilon_{0} \beta_{\|_{0}}, p_{\perp_{0}} c=\varepsilon_{0} \beta_{\perp_{0}}$, and $\sinh ^{-1} y=\ln \left(y+\sqrt{y^{2}+1}\right)$, equation A.15 reduces to:

$$
R=x_{\perp_{\max }}=\frac{\varepsilon_{0} \beta_{\perp_{0}}}{e E} \ln \left(\frac{1+\beta_{\|_{0}}}{1-\beta_{\|_{0}}}\right)
$$

which is the range equation used in the newly-implemented algorithm in BEAMnrc to simulate off-focal radiation. When $\varepsilon_{0}$ is expressed in $\mathrm{MeV}, E$ in $\mathrm{MV} / \mathrm{cm}$ and $e$ in number of elementary charges ( 1 for an electron), the resulting $R$ is in $\mathrm{cm}$. To verify that the derivation presented above is correct, simple algebra shows that for $\beta \ll 1$, equation A.16 reduces to its known non-relativistic limit $\left(R=2 m_{0} v_{\|_{0}} v_{\perp_{0}} / e E\right)$. Another check is to solve for $t$ in equation A.14 and substitute the result into equation A.12 to eliminate $t$. After very tedious algebra, and by introducing $\gamma_{0}=1 / \sqrt{1-\beta_{0}^{2}}$, it can be shown that the trajectory is given by:

$$
x_{\|}=\frac{m_{0} c^{2} \gamma_{0}}{e E}\left[\cosh \left(\frac{e E x_{\perp}}{m_{0} c^{2} \gamma_{0} \beta_{\|_{0}}}\right)-1+\beta_{\|_{0}} \sinh \left(\frac{e E x_{\perp}}{m_{0} c^{2} \gamma_{0} \beta_{\|_{0}}}\right)\right]
$$

which is the same equation given without proof by Bielajew. ${ }^{103}$ With more tedious algebra, it can also be shown that equation A.17 reduces to the familiar parabolic non-relativistic form when $\beta \ll 1$,

$$
\lim _{\beta \ll 1} x_{\|}=v_{\|_{0}} t-\frac{e E}{2 m_{0}} t^{2}
$$

Equation A.17 could have been used directly from Bielajew ${ }^{103}$ to reach equation A.16 by putting $x_{\|}=0$ and solving for $x_{\perp}$. The derivation presented above would still have been needed for more insight about the physics of the problem and, more importantly, to make sure equation A.17 as reported by Bielajew ${ }^{103}$ does not have any typos (in the past, the same author reported an equation involving transport in electromagnetic field with a typo, and he noted the typo elsewhere).

\section{A.1. RELATIVISTIC KINEMATICS OF ELECTRON MOTION}




\section{A.2 Added algorithm in BEAMnrc to transport electrons backscattered from x-ray targets}

In a BEAMnrc simulation of an $\mathrm{x}$-ray tube, the following parameters are known for each electron backscattering out of the target into the vacuum: the total energy $\left(\varepsilon_{0}\right)$, the position of backscatter $\left(x_{0}, y_{0}, z_{0}\right)$ and the direction cosines $\left(u_{0}, v_{0}, w_{0}\right.$, or briefly $\hat{n}_{0}$ ). The goal of the following algorithm is to calculate the same parameters for the backscattered electron at the instant it re-enters the target (i.e. $\left.\varepsilon_{f}, x_{f}, y_{f}, z_{f}, u_{f}, v_{f}, w_{f}\right)$

- For $\varepsilon_{f}$ : In vacuum, conservation of energy dictates that the energy lost by the electron during its deceleration is re-gained during its acceleration, so,

$$
\varepsilon_{f}=\varepsilon_{0}
$$

- For $x_{f}, y_{f}, z_{f}$ : The equation of a line passing through point $\left(x_{0}, y_{0}, z_{0}\right)$ and having direction cosines $\left(u_{0}, v_{0}, w_{0}\right)$ is $\frac{x-x_{0}}{u_{0}}=\frac{y-y_{0}}{v_{0}}=\frac{z-z_{0}}{w_{0}}$. The equation of a plane whose unit normal vector has direction cosines $(\mathrm{a}, \mathrm{b}, \mathrm{c})$ is $a x+b y+c z+d=0$. The x-ray target surface contains the origin $(0,0,0)$, and so $d=0$. If the target angle with respect to $+z$ direction is $\theta$, then the direction cosines of the unit vector $\hat{n}_{1}$ normal to the target surface are $(\cos \theta, 0, \sin \theta)$, and the equation of the plane of the target surface is $\cos \theta x+\sin \theta z=0$. Based on this, the plane of motion of the backscattered electron contains the point $\left(x_{0}, y_{0}, z_{0}\right)$, contains the line $\frac{x-x_{0}}{u_{0}}=\frac{y-y_{0}}{v_{0}}=\frac{z-z_{0}}{w_{0}}$, and is perpendicular to the plane $\cos \theta x+\sin \theta z=0$. The equation of a plane satisfying these conditions is ${ }^{104}$ 


$$
\left|\begin{array}{cccc}
x & y & z & 1 \\
x_{0} & y_{0} & z_{0} & 1 \\
\cos \theta & 0 & \sin \theta & 0 \\
u_{0} & v_{0} & w_{0} & 0
\end{array}\right|=0
$$

which reduces to $P_{1} x+P_{2} y+P_{3} z+P_{4}=0$, where $P_{1}, P_{2}$ and $P_{3}$ are the direction cosines of the unit vector, $\hat{n}_{2}$, normal to the plane of motion, with $P_{1}=-v_{0} \sin \theta / F, P_{2}=\left(u_{0} \sin \theta-w_{0} \cos \theta\right) / F$ and $P_{3}=v_{0} \cos \theta / F$. The factor $F=\sqrt{\left(-v_{0} \sin \theta\right)^{2}+\left(u_{0} \sin \theta-w_{0} \cos \theta\right)^{2}+\left(v_{0} \cos \theta\right)^{2}}$ converts direction numbers of $P_{1}, P_{2}$ and $P_{3}$ into direction cosines. Finally, the equation of the line of intersection $(L)$ between the target surface and the plane of motion of the backscattered electron is $\frac{x-x_{0}}{L_{1}}=\frac{y-y_{0}}{L_{2}}=\frac{z-z_{0}}{L_{3}}$, where $L_{1}, L_{2}$ and $L_{3}$ are the direction cosines of a unit vector, $\hat{n}_{3}$, parallel to $L$. Because $\hat{n}_{3}$ is perpendicular to both $\hat{n}_{1}$ and $\hat{n}_{2}$, its direction cosines $L_{1}, L_{2}$ and $L_{3}$ can be obtained from the cross product of $\hat{n}_{1}$ and $\hat{n}_{2}$, i.e.

$\hat{n}_{3}=\hat{n}_{1} \times \hat{n}_{2}=\left|\begin{array}{ccc}\hat{x} & \hat{y} & \hat{z} \\ P_{1} & P_{2} & P_{3} \\ \cos \theta & 0 & \sin \theta\end{array}\right|=P_{2} \sin \theta \hat{x}+\left(P_{3} \cos \theta-P_{1} \sin \theta\right) \hat{y}-P_{2} \cos \theta \hat{z}$ Then, $L_{1}=P_{2} \sin \theta, L_{2}=P_{3} \cos \theta-P_{1} \sin \theta=v_{0} / F$ when reduced, and $L_{3}=$ $-P_{2} \cos \theta$. Knowing the range $R$ from equation A.16 in appendix A.1, and the direction cosines of $\hat{n}_{3}$, the backscattered electron can be transported along the line $L$ to its location of re-entry:

$$
x_{f}=x_{0}+L_{1} R, \quad y_{f}=y_{0}+L_{2} R, \quad z_{f}=z_{0}+L_{3} R
$$

Since the electron can be transported either way along $L$, certain checks are done to ensure that the transport is in the proper direction. Next, $x_{f}, y_{f}$ and $z_{f}$ are used

\section{A.2. TRANSPORT OF BACKSCATTERED ELECTRONS}


to check if the re-entry location of the backscattered electron is outside the target dimensions. If so, the electron is discarded. Otherwise, the algorithm proceeds to calculate the final direction cosines $\left(u_{f}, v_{f}, w_{f}\right)$ of the returning backscattered electron.

- For $u_{f}, v_{f}, w_{f}$ : The angle of backscattering, $\alpha$, is the angle between $\hat{n}_{0}$ and $\hat{n}_{3}$, which are both contained in the plane of motion. $\alpha$ is also the angle of re-entry, which is the angle between a unit vector, $\hat{n}_{f}$, with direction cosines $\left(u_{f}, v_{f}, w_{f}\right)$ and $\hat{n}_{3}$. $\alpha$ can easily be calculated using the dot product of either $\hat{n}_{0}$ and $\hat{n}_{3}$ or $\hat{n}_{f}$ and $\hat{n}_{3}$. In the plane of motion of the backscattered electron, $\hat{n}_{f}$ can be broken down (with respect to the electric field) into a parallel and a perpendicular component. The parallel component has the same direction cosines as $\hat{n}_{1}$ but with a negative sign and weighted by $\sin \alpha$. The perpendicular component has the same direction cosines as $\hat{n}_{3}$ without change of $\operatorname{sign}$ but weighted by $\cos \alpha$. The final direction cosines are the algebraic sum of these components.

$$
u_{f}=L_{1} \cos \alpha-\cos \theta \sin \alpha, \quad v_{f}=L_{2} \cos \alpha=v_{0}, \quad w_{f}=L_{3} \cos \alpha-\sin \theta \sin \alpha
$$

Algebraic manipulation reduces $L_{2} \cos \alpha$ to $v_{0}$. This is expected because the y-axis is contained in the plane of target surface.

Equations A.19, A.20 and A.21 determine the parameters needed when the backscattered electron re-enters the target in the simulation. Once the backscattered electron re-enters the target, the simulation proceeds normally. 


\section{References}

[1] E. S. M. Ali and D. W. O. Rogers, Efficiency improvements of x-ray simulations in EGSnrc user-codes using Bremsstrahlung Cross Section Enhancement (BCSE), Med. Phys. 34, 2143 - 2154 (2007).

[2] E. S. M. Ali and D. W. O. Rogers, Benchmarking EGSnrc in the kilovoltage range against experimental measurements of charged particle backscatter coefficient, Phys. Med. Biol., to be submitted (September 2007).

[3] E. S. M. Ali and D. W. O. Rogers, Energy spectra and angular distribution of charged particles backscattered from solid targets, J. Phys. D: Appl. Phys. , to be submitted (September 2007).

[4] E. S. M. Ali and D. W. O. Rogers, Effect of off-focal (extra-focal) radiation on the output parameters of x-ray tubes, Med. Phys. , in preparation (2007).

[5] I. Kawrakow and D. W. O. Rogers, The EGSnrc Code System: Monte Carlo simulation of electron and photon transport, NRC Technical Report PIRS-701 v4-2-2-5, National Research Council of Canada, Ottawa, Canada. http://www.irs.inms.nrc.ca/inms/irs/EGSnrc/EGSnrc.html, 2007.

[6] D. W. O. Rogers, B. Walters, and I. Kawrakow, BEAMnrc Users Manual, NRC Technical Report PIRS-509(A) rev $\mathrm{K}$, National Research Council of Canada, Ottawa, Canada. http://www.irs.inms.nrc.ca/BEAM/beamhome.html, 2007.

[7] I. Kawrakow, Accurate condensed history Monte Carlo simulation of electron transport. I. EGSnrc, the new EGS4 version, Med. Phys. 27, 485 - 498 (2000).

[8] R. L. Ford and W. R. Nelson, The EGS code system - Version 3, Report SLAC-210, Stanford Linear Accelerator Center, Stanford, California, 1978. 
[9] I. Kawrakow, Electron impact ionization cross sections for EGSnrc, Med. Phys. (abstract) 29, 1230 (2002).

[10] M. J. Berger and J. H. Hubbell, XCOM: Photon cross sections on a personal computer, Report NBSIR87-3597, NIST, Gaithersburg, MD 20899, U.S.A., 1987.

[11] S. M. Seltzer and M. J. Berger, Bremsstrahlung spectra from electron interactions with screened atomic nuclei and orbital electrons, Nucl. Inst. Meth. Phys. Res. B 12, 95 - 134 (1985).

[12] S. M. Seltzer and M. J. Berger, Bremsstrahlung energy spectra from electrons with kinetic energy from $1 \mathrm{keV}$ to $10 \mathrm{GeV}$ incident on screened nuclei and orbital electrons of neutral atoms with $\mathrm{Z}=1-100$, Atomic Data and Nuclear Data Tables 35, 345-418 (1986).

[13] I. Lux and L. Koblinger, Monte Carlo particle transport methods: neutron and photon calculations, CRC Press, New York, 1991.

[14] W. R. Nelson, H. Hirayama, and D. W. O. Rogers, The EGS4 code system, Report SLAC-265, Stanford Linear Accelerator Center, Stanford, California, 1985.

[15] D. W. O. Rogers, B. A. Faddegon, G. X. Ding, C.-M. Ma, J. Wei, and T. R. Mackie, BEAM: A Monte Carlo code to simulate radiotherapy treatment units, Med. Phys. 22, 503 - 524 (1995).

[16] I. Kawrakow, D. W. O. Rogers, and B. Walters, Large efficiency improvements in BEAMnrc using directional bremsstrahlung splitting, Med. Phys. 31, $2883-2898$ (2004).

[17] E. Mainegra-Hing and I. Kawrakow, Efficient x-ray tube simulations, Med. Phys. 33, 2683 - 2690 (2006).

[18] J. M. Boone and J. A. Seibert, An accurate method for computer-generating tungsten anode x-ray spectra from 30 to $140 \mathrm{kV}$, Med. Phys. 24, 1661 1670 (1997).

[19] F. Verhaegen, A. E. Nahum, S. Van de Putte, and Y. Namito, Monte Carlo modelling of radiotherapy kV x-ray units, Phys. Med. Biol. 44, $1767-1789$ (1999). 
[20] M. Bhat, J. Pattison, G. Bibbo, and M. Caon, Off-axis x-ray spectra: A comparison of Monte Carlo simulated and computed spectra with measured spectra, Med. Phys. 26, 303 - 309 (1999).

[21] L. M. N. Tavora, E. J. Morton, F. P. Santos, and T. H. V. T. Dias, Simulation of $\mathrm{x}$-ray tubes for imaging applications, IEEE Trans. on Nuclear Science 47, $1493-1498$ (2000).

[22] F. Verhaegen and I. A. Castellano, Microdosimetric characterisation of 28 $\mathrm{kVp}$ MoMo RhRh RhAl WRh and MoRh mammography x-ray spectra, Radiat. Prot. Dosim. 99, 393 - 396 (2002).

[23] L. Ben-Omrane, F. Verhaegen, N. Chahed, and S. Mtimet, An investigation of entrance surface dose calculations for diagnostic radiology using Monte Carlo simulations and radiotherapy dosimetry formalisms, Phys. Med. Biol. 48, 1809 - 1824 (2003).

[24] M. A. Ebert and B. Carruthers, Dosimetric characteristics of a low-kV intraoperative $\mathrm{x}$-ray source: Implications for use in a clinical trial for treatment of low-risk breast cancer, Med. Phys. 30, 2424-2431 (2003).

[25] F. Verhaegen, B. Reniers, F. Deblois, S. Devic, J. Seuntjens, and D. Hristov, Dosimetric and microdosimetric study of contrast-enhanced radiotherapy with kilovolt x-rays, Phys. Med. Biol. 50, 3555-3569 (2005).

[26] H. Deloar et al, Investigations of different kilovoltage x-ray energy for threedimensional converging stereotactic radiotherapy system: Monte Carlo simulation with CT data, Med. Phys. 33, 4635-4642 (2006).

[27] G. Jarry, S. A. Graham, D. J. Moseley, D. J. Jaffray, J. H. Siewerdsen, and F. Verhaegen, Characterization of scattered radiation in $\mathrm{kV}$ CBCT images using Monte Carlo simulations, Med. Phys. 33, 4320 - 4329 (2006).

[28] R. E. P. Taylor, G. Yegin, and D. W. O. Rogers, Monte Carlo modeling of the Xoft Axxent x-ray source, Med. Phys. 33, 2205 (abs) (2006).

[29] G. X. Ding, D. M. Duggan, and C. W. Coffey, Characteristics of kilovoltage $\mathrm{x}$-ray beams used for cone-beam computed tomography in radiation therapy, Phys. Med. Biol. 52, 1595 - 1615 (2007).

[30] R. Hill, B. Healy, L. Holloway, and C. Baldock, An investigation of dose changes for therapeutic kilovoltage $\mathrm{x}$-ray beams with underlying lead shielding, Med. Phys. 34, 3045 - 3053 (2007). 
[31] E. O. Agyingi, P. N. Mobit, and G. A. Sandison, Energy response of an aluminum oxide detector in kilovoltage and megavoltage photon beams: An EGSnrc Monte Carlo simulation study, Radiat. Prot. Dosim. 118, $28-31$ (2005).

[32] M. C. Azner, J. Medin, B. Hemdal, A. T. King, L. Botter-Jensen, and S. Mattsson, A Monte Carlo study of the energy dependence of $\mathrm{Al}_{2} \mathrm{O}_{3}: \mathrm{C}$ crystals for real-time in vivo dosimetry, Radiat. Prot. Dosim. 114, 444 449 (2005).

[33] G. G. Zeng and J. P. McCaffrey, The response of alanine to a $150 \mathrm{keV} x$-ray beam, Rad. Phys. Chem. 72, 537 - 540 (2005).

[34] D. J. La Russa and D. W. O. Rogers, An EGSnrc investigation of the $P_{T P}$ correction factor for ion chambers in kilovoltage x-rays, Med. Phys. 33, $4590-4599$ (2006).

[35] M. Berger and S. Seltzer, ETRAN Monte Carlo code system for electron and photon transport through extended media, Report CCC-107, Radiation Shielding Information Centre, ORNL, Oak Ridge, Tennessee (1973).

[36] F. Salvat, J. M. Fernandez-Varea, J. Baro, and J. Sempau, PENELOPE, a code system for Monte Carlo simulation of electron and photon transport, University of Barcelona Report (2003).

[37] E. Acosta, X. Llovet, E. Coleoni, and J. Riveros, Monte Carlo simulation of x-ray emission by kilovolt electron bombardment, J. Appl. Phys. 83, 6038 - 6049 (1998).

[38] B. R. B. Walters, I. Kawrakow, and D. W. O. Rogers, History by history statistical estimators in the BEAM code system, Med. Phys. 29, 2745 2752 (2002).

[39] I. Kawrakow, On the efficiency of photon beam treatment head calculations, Med. Phys. 32, 2320 - 2326 (2005).

[40] R. E. P. Taylor, G. Yegin, and D. W. O. Rogers, Benchmarking BrachyDose: voxel-based EGSnrc Monte Carlo calculations of TG-43 dosimetry parameters, Med. Phys. 34, $445-457$ (2007).

[41] M. J. Rivard, S. D. Davis, L. A. DeWerd, T. W. Rusch, and S. Axelrod, Calculated and measured brachytherapy dosimetry parameters in water for

REFERENCES 
the Xoft Axxent X-Ray Source: An electronic brachytherapy source, Med. Phys. 33, $4020-4032$ (2006).

[42] G. Yegin, A new approach to geometry modelling of Monte Carlo particle transport: an application to EGS, Nucl. Inst. Meth. B 211, $331-338$ (2003).

[43] D. Sheikh-Bagheri and D. W. O. Rogers, Calculation of nine megavoltage photon beam spectra using the BEAM Monte Carlo code, Med. Phys. 29, $391-402$ (2002).

[44] J. Borg, I. Kawrakow, D. W. O. Rogers, and J. P. Seuntjens, Experimental verification of EGSnrc Monte Carlo calculated ion chamber response in low energy photon beams, in Proc. 22nd Ann. Int. Conf. of IEEE Engineering in Medicine and Biology Society, pages 3152 - 3155, Vol. 4, IEEE, 2000.

[45] I. Kawrakow and D. W. O. Rogers, The EGSnrc System, a status report, in Advanced Monte Carlo for Radiation Physics, Particle Transport Simulation and Applications: Proc. of the Monte Carlo 2000 Meeting Lisbon, edited by A. Kling, F. Barao, M. Nakagawa, L. Távora, and P. Vaz, pages $135-140$, Springer, Berlin, 2001.

[46] F. Verhaegen, Evaluation of the EGSnrc Monte Carlo code for interface dosimetry near high-Z media exposed to kilovolt and ${ }^{60}$ Co photons, Phys. Med. Biol. 47, 1691 - 1705 (2002).

[47] Z. Wen, R. Fahrig, S. Conolly, and N. J. Pelc, Investigation of electron trajectories of an x-ray tube in magnetic fields of MR scanners, Med. Phys. 34, $2048-2058$ (2007).

[48] Z. Wen, N. J. Pelc, W. R. Nelson, and R. Fahrig, Study of increased radiation when an x-ray tube is placed in a strong magnetic field, Med. Phys. 34, $408-418$ (2007).

[49] F. A. Buffa and F. Verhaegen, Backscatter and dose perturbations for lowto medium-energy electron point sources at the interface between materials with different atomic numbers, Radiat. Res. 162, 693 - 701 (2004).

[50] H. Niedrig, Electron backscattering from thin films, J. Appl. Phys. 53, R15 - R49 (1982).

[51] E. H. Darlington, Backscattering of 10-100 keV electrons from thick targets, J. Phys. D: Appl. Phys. 8, 85 - 93 (1975). 
[52] A. Darlinski, Measurements of angular distribution of the backscattered electrons in the energy range of 5 to $30 \mathrm{keV}$, Phys. Status Solidi A 63, 663 -668 (1981).

[53] H. E. Bishop, Some electron backscattering measurements for solid targets, in Proc. 4th Int. Conf. on X-ray Optics and X-ray Microanalysis, edited by R. Castaing, P. Deschamps, and J. Philibert, pages 153 - 158, Hermann, Paris, 1965.

[54] P. G. Coleman, L. Albercht, K. O. Jensen, and A. B. Walker, Positron backscattering from elemental solids, J. Phys.: Condens. Matter 4, 10311 10322 (1992).

[55] V. E. Coslett and R. N. Thomas, Multiple scattering of 5-30 keV electrons in evaporated metal films III: Backscattering and absorption, Brit. J. Appl. Phys. 16, 779 - 796 (1965).

[56] H. Drescher, L. Reimer, and H. Seidel, Backscattering and secondary electron emission of 10-100 keV and correlation to scanning electron microscopy (in German), Z. Angew. Phys. 29, $331-336$ (1970).

[57] H. J. Fitting and R. Technow, Electron backscattering at various angles of incidence, Phys. Status Solidi A 76, K151 - K154 (1983).

[58] P. Gérard, J. L. Balladore, J. P. Martinez, and A. Ouabbou, Experimental determination of angular-energy distributions of electrons backscattered by bulk gold and silicon samples, Scanning 17, 377 - 386 (1995).

[59] K. F. J. Heinrich, Electron probe microanalysis by specimen current measurements, in Proc. 4th Int. Conf. on X-ray Optics and X-ray Microanalysis, edited by R. Castaing, P. Deschamps, and J. Philibert, pages 159 - 167, Hermann, Paris, 1965.

[60] H. J. Hunger and L. Küchler, Measurements of the electron backscattering coefficient for quantitative EPMA in the energy range of 4 to $40 \mathrm{keV}$, Phys. Status Solidi A 56, K45 - K48 (1979).

[61] H. Kanter, On the backscattering of electrons in the energy range of 10 to $100 \mathrm{keV}$ (in German), Ann. Phys. (Leipzig) 20, 144- 166 (1957).

[62] H. Kanter, Backscattering of kilovolt electrons from thin films, Brit. J. Appl. Phys. 15, 555 - 559 (1964). 
[63] H. Kulenkampff and K. Rüttiger, Energy and angular distribution of backscattered electrons (in German), Z. Phys. 137, 426 - 434 (1954).

[64] H. Kulenkampff and W. Spyra, Energy distribution of backscattered electrons (in German), Z. Phys. 137, 416 - 425 (1954).

[65] J. Mäkinen, S. Palko, J. Martikainen, and P. Hautojärvi, Positron backscattering probabilities from solid surfaces at $2-30 \mathrm{keV}$, J. Phys.: Condens. Matter 4, L503 - L508 (1992).

[66] J. W. Martin, J. Yuan, M. J. Betancourt, B. W. Filippone, S. A. Hoedl, T. M. Ito, B. Plaster, and A. R. Young, New measurements and quantitative analysis of electron backscattering in the energy range of neutron $\beta$ decay, Phys. Rev. C 73, 015501(6) (2006).

[67] G. R. Massoumi, N. Hozhabri, W. N. Lennard, and P. J. Schultz, Doubly differential positron-backscattering yields, Phys. Rev. B 44, 3486 - 3489 (1991).

[68] G. R. Massoumi, W. N. Lennard, and P. J. Schultz, Electron and positron backscattering in the medium-energy range, Phys. Rev. B 47, $11007-11018$ (1993).

[69] T. Matsukawa, R. Shimizu, and H. Hashimoto, Measurements of the energy distribution of backscattered kilovolt electrons with a spherical retardingfield energy analyser, J. Phys. D: Appl. Phys. 7, 695 - 702 (1974).

[70] G. Neubert and S. Rogaschewski, Backscattering coefficient measurements of 15 to $60 \mathrm{keV}$ electrons for solids at various angles of incidence, Phys. Status Solidi A 59, 35 - 41 (1980).

[71] H. Niedrig and P. Sieber, Backscattering of medium/fast electrons by thin films (in German), Z. Angew. Phys. 31, 27 - 31 (1971).

[72] E. Rau, H. Hoffmeister, R. Sennov, and H. Kohl, Comparison of experimental and Monte Carlo simulated BSE spectra of multilayered structures and 'in-depth' measurements in a SEM, J. Phys. D: Appl. Phys. 35, 1433 1437 (2002).

[73] G. Soum, H. Ahmed, F. Arnal, B. Jouffrey, and P. Verdier, Measurement of transmission and backscatter coefficients of monoenergetic electrons (0.05-3 $\mathrm{MeV}$ ) (in French), J. Microsc. Spect. Elec. 9, 1 - 16 (1984).

REFERENCES 
[74] E. Weinryb and J. Philibert, Measurement of backscatter coefficients of electrons from 5 to $30 \mathrm{keV}$ (in French), C. R. Acad. Sci. (Paris) 258, 4535 -4538 (1964).

[75] D. B. Wittry, Secondary electron emission in the electron probe, in Proc. 4th Int. Conf. on X-ray Optics and X-ray Microanalysis, edited by R. Castaing, P. Deschamps, and J. Philibert, pages 168 - 180, Hermann, Paris, 1965.

[76] http://physics.nist.gov.

[77] C. Malamut, D. W. O. Rogers, and A. F. Bielajew, Calculation of water/air stopping-power ratios using EGS4 with explicit treatment of electron - positron differences, Med. Phys. 18, 1222 - 1228 (1991).

[78] R. Thoraeus, The amount of off-focus radiation in beams from various types of Roentgen tubes, Acta Radiol. 18, 753 - 760 (1937).

[79] R. F. Wagner, K. E. Weaver, E. W. Denny, and R. G. Bostrom, Towards a unified view of radiological imaging systems - Part I: Noiseless images, Med. Phys. 1, $11-24$ (1974).

[80] M. Braun, Focal spots in the future of mammography, in Reduced-Dose Mammography: Proc. 2nd Reduced-Dose Mammography Meeting, Roswell Park Institute, Buffalo, NY, Oct. 1978, edited by W. W. Logan and E. P. Muntz, pages 195 - 209, Masson Publishing USA Inc., New York, 1979.

[81] W. W. Roeck, X-ray source assembly acceptance testing, in Specifications, acceptance testing and quality control of diagnostic x-ray imaging equipment, Medical Physics Monograph No. 20, edited by J. A. Seibert, G. T. Barnes, and R. G. Gould, pages 205 - 231, AAPM, New York, 1994.

[82] H. Kuhn and H. Gajewski, The extra-focal radiation from rotating anode $\mathrm{x}$-ray tubes and its effect on the quality of the radiograph, Electromedica $4,125-130$ (1971).

[83] G. Rao, Influence of off-focal radiation on radiographic resolution and patient exposure, Appl. Radiol. , 45 - 49, May/June (1974).

[84] W. Jackson, Measurement of the kilovoltage by the penetrameter - Theoretical aspects, Phys. Med. Biol. 20, 268 - 281 (1975). 
[85] K. E. Weaver, M. S. Robert, and F. Wagner, Performance considerations of x-ray tube focal spot, SPIE - Medical X-ray Photo-Optical Systems Evaluation 56, 150 - 158 (1975).

[86] R. Birch, The spectrum and intensity of extra-focal (off-focus) radiation, Brit. J. Radiol. 49, $951-955$ (1976).

[87] H. E. Johns and J. R. Cunningham, The Physics of Radiology, Fourth edition, Charles C. Thomas, Springfield, Illinois, 1983.

[88] W. W. Roeck, J. T. Martin, and R. Beach, Analysis of contributing factors to the occurrence of off-focus radiation (OFR), Med. Phys. 19, $1201-1204$ (1992).

[89] K. Cranley, B. J. Gilmore, G. W. Fogarty, and L. Desponds, Catalogue of diagnostic x-ray spectra and other data, CD edition 1997, electronic version prepared by D Sutton, Technical Report Report 78, The Institute of Physics and Engineering in Medicine (IPEM), York, 1997.

[90] S. Z. Shen, A. K. Bloomquist, G. E. Mawdsley, and M. J. Yaffe, Effect of scatter and an antiscatter grid on the performance of a slot-scanning digital mammography system, Med. Phys. 33, 1108 - 1115 (2006).

[91] E. Gabbay, X-ray source assembly design, in Specifications, acceptance testing and quality control of diagnostic x-ray imaging equipment, Medical Physics Monograph No. 20, edited by J. A. Seibert, G. T. Barnes, and R. G. Gould, pages 173 - 204, AAPM, New York, 1994.

[92] G. V. Pavlinsky and A. Y. Portnoy, Calculating the spectral distribution of $x$-ray tubes with grounded cathode, Rad. Phys. Chem. 62, $207-213$ (2001).

[93] G. M. Ardran and H. E. Crooks, Penetrameter cassette calibration to 400 $\mathrm{kV}$ and effects of extra-focal radiation when measuring tube filtration, Brit. J. Radiol. 51, 29 - 34 (1978).

[94] H. D. Nagel, Limitations in the determination of total filtration of x-ray tube assemblies, Phys. Med. Biol. 33, 271 - 289 (1988).

[95] C. A. Carlsson, Differences in reported backscatter factors for low-energy x-rays: a literature study, Phys. Med. Biol. 38, 521 - 531 (1993).

REFERENCES 
[96] O. Mattsson, Some factors which influence the quality of the radiographic image, Acta Radiol., Suppl 120: Practical photographic problems in radiography , $147-206$ (1955).

[97] K. Shiga and M. Yoshida, Methods of eliminating off-focus x-rays in rotating-anode x-ray tubes, Toshiba Review , 41 - 48 (Winter 1963).

[98] S. Agostinelli et al., GEANT4 - a simulation toolkit, Nucl. Inst. Meth. A 506, $250-303$ (2003).

[99] J. Rodney and M. Vaughan, Electron flow in the conventional x-ray tube, IEEE Trans. Electron Dev. 32, 654 - 657 (1985).

[100] P. Schardt, J. Deuringer, J. Freudenberger, E. Hell, and W. Knüpfer, New xray tube performance in computed tomography by introducing the rotating envelope tube technology, Med. Phys. 31, 2699 - 2706 (2004).

[101] G. V. Pavlinsky and A. Y. Portnoy, Formation features of radiation from X-ray tubes with grounded cathode, X-Ray Spectrom. 31, 247 - 251 (2002).

[102] R. Nowotny and K. H. Meghzifene, Simulation of the effect of anode surface roughness on diagnostic x-ray spectra, Phys. Med. Biol. 47, 3973 - 3983 (2002).

[103] A. F. Bielajew, Electron Transport in $\vec{E}$ and $\vec{B}$ Fields, in Monte Carlo Transport of Electrons and Photons, edited by T. M. Jenkins, W. R. Nelson, A. Rindi, A. E. Nahum, and D. W. O. Rogers, pages 421 - 434, Plenum Press, New York, 1988.

[104] G. Salmon, A treatise on the analytic geometry of three dimensions, Vol. I, 7th Ed., Chelsea Publishing Co., New York, 1965. 